\title{
Modulation of gene expression and DNA adduct formation by chlorophyllin in human mammary cells exposed to benzopyrenes
}

Kaarthik John

West Virginia University

Follow this and additional works at: https://researchrepository.wvu.edu/etd

\section{Recommended Citation}

John, Kaarthik, "Modulation of gene expression and DNA adduct formation by chlorophyllin in human mammary cells exposed to benzopyrenes" (2006). Graduate Theses, Dissertations, and Problem Reports. 2439.

https://researchrepository.wvu.edu/etd/2439

This Dissertation is protected by copyright and/or related rights. It has been brought to you by the The Research Repository @ WVU with permission from the rights-holder(s). You are free to use this Dissertation in any way that is permitted by the copyright and related rights legislation that applies to your use. For other uses you must obtain permission from the rights-holder(s) directly, unless additional rights are indicated by a Creative Commons license in the record and/ or on the work itself. This Dissertation has been accepted for inclusion in WVU Graduate Theses, Dissertations, and Problem Reports collection by an authorized administrator of The Research Repository @ WVU.

For more information, please contact researchrepository@mail.wvu.edu. 


\title{
MODULATION OF GENE EXPRESSION AND DNA ADDUCT FORMATION BY CHLOROPHYLLIN IN HUMAN MAMMARY CELLS EXPOSED TO BENZOPYRENES
}

\section{Kaarthik John}

Dissertation submitted to the

Davis College of Agriculture and Forestry and Consumer Sciences at West Virginia University in partial fulfillment of the requirements for the degree of

\section{Doctor of Philosophy in Genetics and Developmental Biology}

\author{
Joginder Nath, Ph.D., Chair, \\ Ainsley Weston, Ph.D. \\ Dale Karlson, Ph.D. \\ Jed H. Doelling, Ph.D. \\ Matthew E. Wilson, Ph.D.
}

Department of Plant and Soil Sciences

Morgantown, West Virginia

2006

Keywords: Cytochrome P450, benzopyrene, chlorophyllin, chemoprevention, DNA adducts 


\title{
ABSTRACT \\ Modulation of gene expression and DNA adduct formation by chlorophyllin in human mammary cells exposed to benzopyrenes
}

\author{
Kaarthik John
}

This study investigated metabolic activation by benzopyrenes $(\mathrm{BP}$ and $\mathrm{B}[\mathrm{e}] \mathrm{P})$ in human mammary cells and modulation by chlorophyllin (CHL, a chemopreventive agent). Among 6 NHMEC strains monitored using microarrays, 54 genes were up-regulated and 11 down-regulated by signal $\log$ ratio (SLR) $\geq 1.5$ on treatment with BP alone. Pre CHL + post $\mathrm{BP}+\mathrm{CHL}$ treatment up-regulated the expression of 129 genes and down-regulated those of 35 genes by SLR $\geq 1.5$. Studies on CYP1 gene induction and BP-DNA adduct formation among $20 \mathrm{NHMECs}$ revealed wide inter-individual variations both in the induction (3-96-fold for $C Y P 1 A 1$ and 4-43-fold for $C Y P 1 B 1$, respectively) and modulation (2-54-fold and 1-39-fold for $C Y P 1 A 1$ and $C Y P 1 B 1$, respectively) of $C Y P 1$ gene expression and reduction of $\mathrm{BP}-\mathrm{DNA}$ adduct formation $(0 \%$ to $86 \%)$ on treatment with $\mathrm{BP} \pm \mathrm{CHL}$. $\mathrm{B}[\mathrm{e}] \mathrm{P}$ was a very poor inducer of $C Y P 1$ gene expression and also exhibited no detectable adduct formation among the 2 NHMEC strains used, when compared to BP. There was a reduction of BP induced $C Y 1 A 1$ ( $0-40 \%$ among the 2 cell strains) and $C Y P 1 B 1$ expression (5-50\% among the 2 cell strains) across all CHL treatments except for pre CHL+ post BP treatment and pre $\mathrm{CHL}+$ post $\mathrm{BP}+\mathrm{CHL}$ treatment in one of the cell strains. CHL enhanced $\mathrm{B}[\mathrm{e}] \mathrm{P}$ induced $C Y P 1$ gene expression on treatment of cells with $\mathrm{B}[\mathrm{e}] \mathrm{P}+\mathrm{CHL}$ and pre $\mathrm{CHL}$ + post $\mathrm{B}[\mathrm{e}] \mathrm{P}+\mathrm{CHL}$. When MCF-7 cells were compared to NHMECs, though basal $C Y P 1 B 1$ expression was nearly 348 times that of $C Y P 1 A 1, \mathrm{MCF}-7$ cells exhibited highly inducible $C Y P 1 A 1$ expression (114 fold) compared to CYP1B1 expression (5 fold). None of the different CHL treatments modulated $C Y P 1$ gene expression or BP-DNA adduct formation in MCF-7 cells as opposed to NHMECs. When MCF-7 cells were compared to $\mathrm{M} 00012$, a NHMEC, across a range of CHL concentrations, only $3 \mu \mathrm{M}, 4 \mu \mathrm{M}$ and $16 \mu \mathrm{M}$ CHL mitigated CYP1 expression to different extents. BP-DNA adduct levels were unaltered in MCF-7 cells but reduced in concentration dependent manner in M00012. These studies show the wide inter-individual variability in response to carcinogens and chemopreventive agents which are to be accounted for while designing intervention strategies. 
Dedicated to my Parents and Sisters. 


\section{ACKNOWLEDGEMENTS}

I consider myself rather privileged to have come under the mentorship of Dr. Joginder Nath, major advisor and Chair of my committee. I would like to thank you for playing an instrumental role in honing me towards being a more accomplished person, both, professionally and personally. I would also like to acknowledge your time, constant guidance and unflinching support.

This work would not have been a reality but for the opportunity given by Dr. Ainsley Weston, co-advisor of my committee, to work in his lab at NIOSH. I value your scientific forethought, constructive criticisms and at times quest for perfectionism in work, all of which will hopefully stand me in good stead in future too.

Most of the work on DNA adduct analysis would not have been possible but for the wonderful collaborative support offered by Dr. Miriam Poirier, head of the carcinogen-DNA interactions unit, NCI, NIH. I would like to thank you not just helping make my dissertation more meaningful but also for helping me out on various counts on various other occasions.

Memories of my early days in the US and my initial years of research at NIOSH wouldn't be complete without my 'mixed' experiences of my interactions with Dr. Channa Keshava, a friend and 'practical research guide'. I am grateful for everything received from you and your family.

The essence of having a more 'complete' and error free dissertation distills from intellectual input, thoughts and suggestions from various qualified sources capable of taking a different perspective of the same problem. I would like to thank my Committee members, Dr. Jed Doelling, Dr. Mathew Wilson and Dr. Dale Karlson for being pivotal in serving these functions.

Credit is due to Dr. Rao Divi, Christine, Marie, Margaret and various other folks at Poirier's lab, NCI for helping me out with the adduct analysis and also teaching me the assay.

Thanks to a post doc Maureen Gwinn, technicians Bonnie Frye, James Ensey and Diana Whipkey, and an undergraduate student, Lora Tennant, at Dr. Weston's lab for 
everything ranging from occasional trouble shooting tips to lively conversations to ease out the fatigue of 'research blues'.

I also appreciate the help and support of various friends and labs at NIOSH and WVU.

Finally, I don't think I would have been able to dream big and get this far in life without constant moral support, inspiration and encouragement from my family. I would like to sincerely thank my parents and my family for everything. 


\section{TABLE OF CONTENTS}

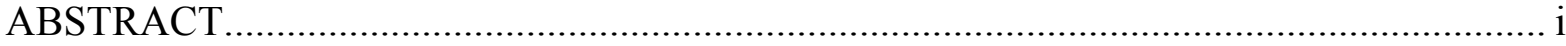

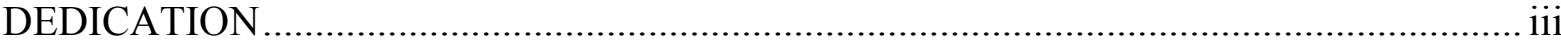

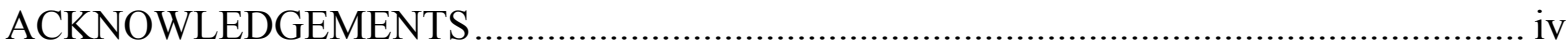

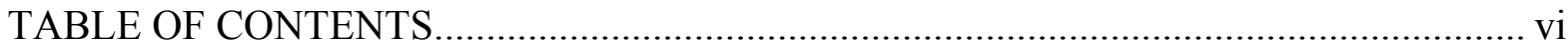

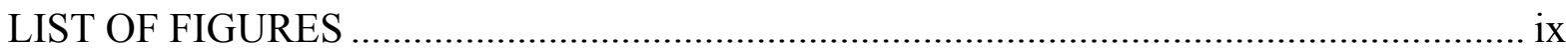

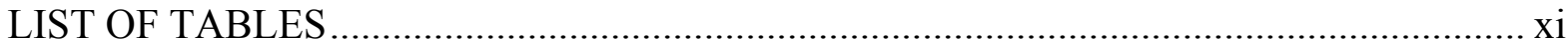

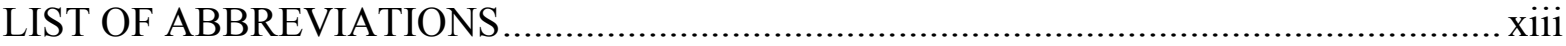

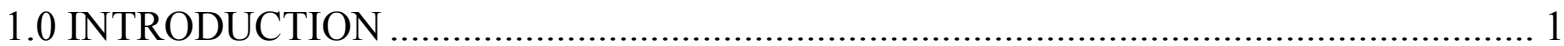

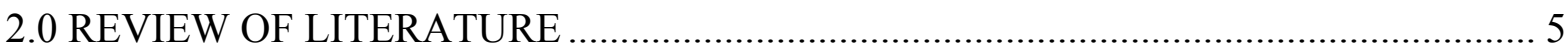

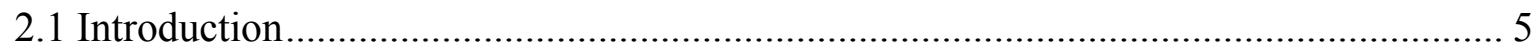

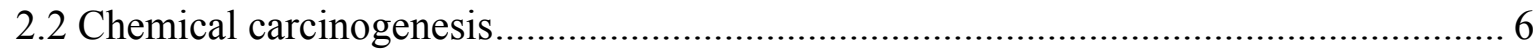

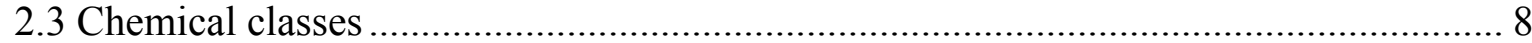

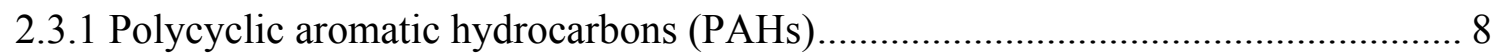

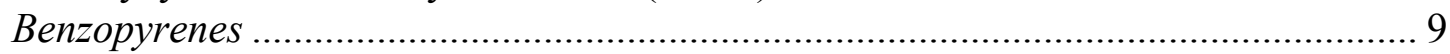

2.3.2 Aromatic amines and heterocyclic amines .................................................. 10

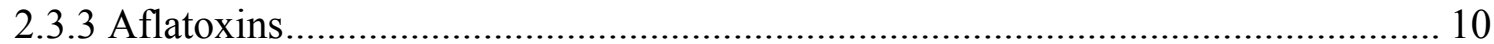

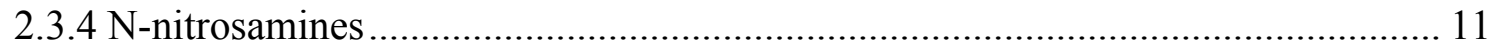

2.3.5 Non-genotoxic agents ............................................................................... 11

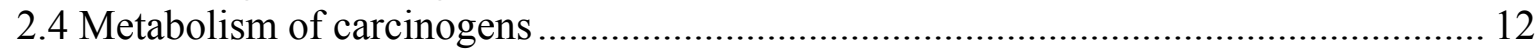

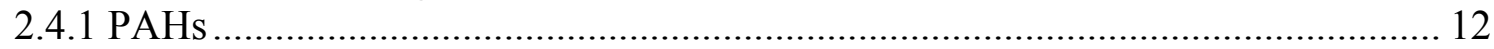

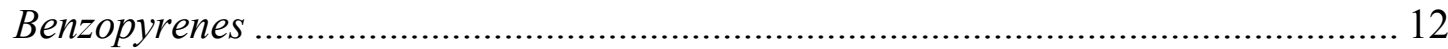

2.4.2 Aromatic amines and heterocyclic amines .................................................... 18

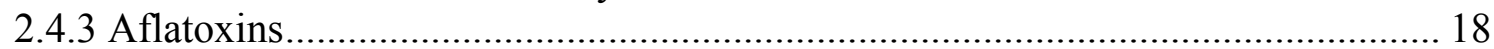

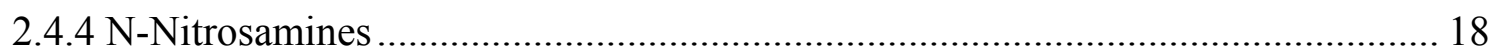

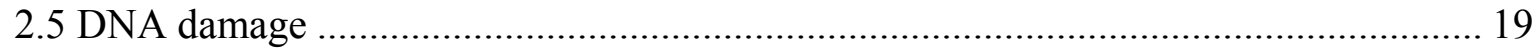

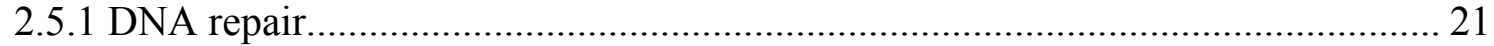

2.6 Potential impact on hormonal carcinogenesis......................................................... 22

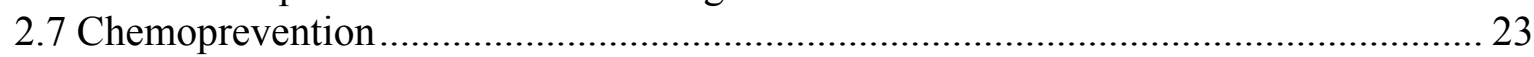

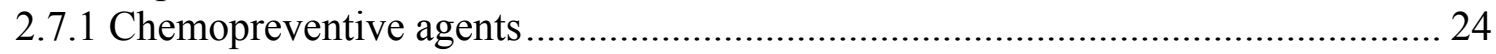

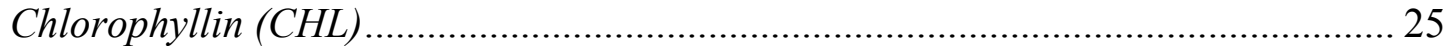

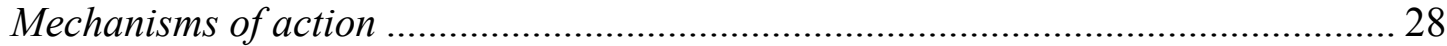

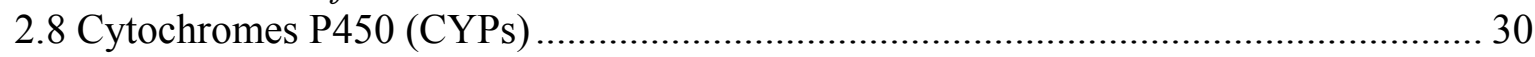

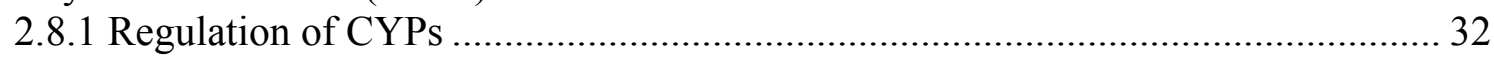

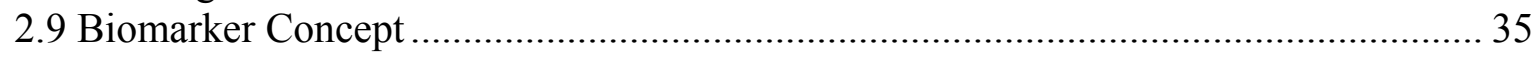

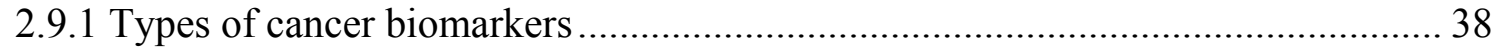

2.9.2 Challenges in using biomarkers ................................................................... 40

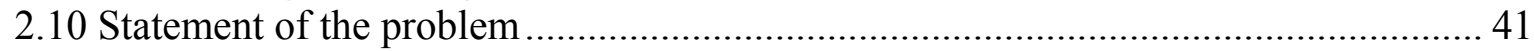

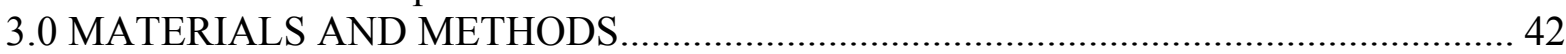

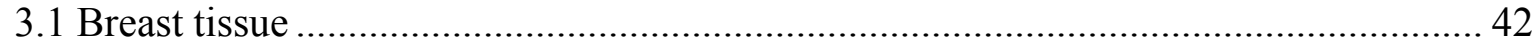

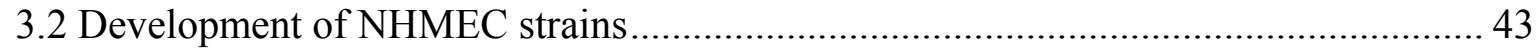

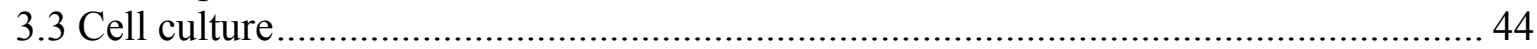

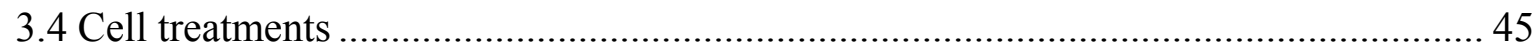

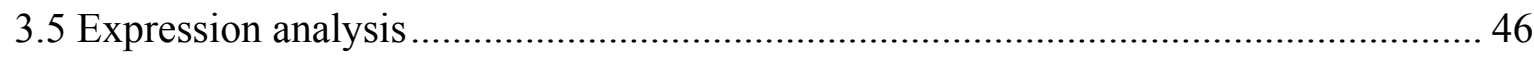

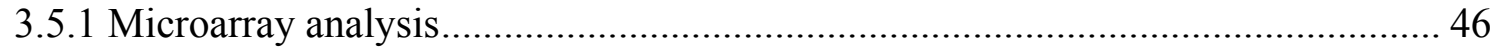




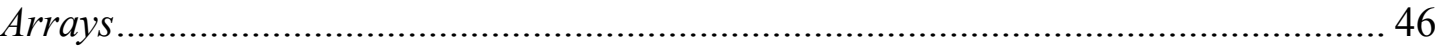

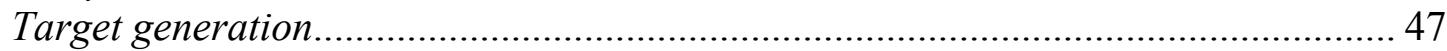

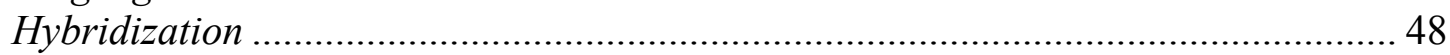

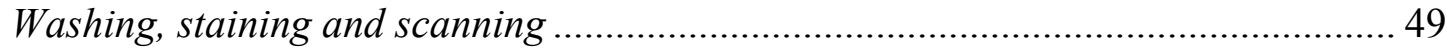

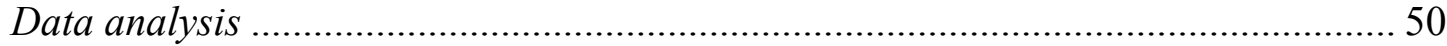

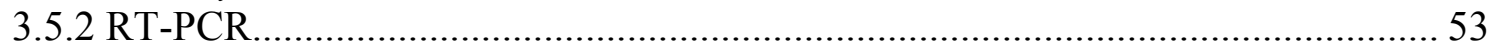

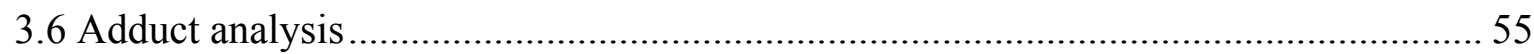

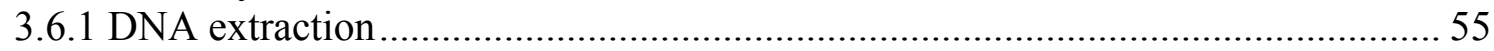

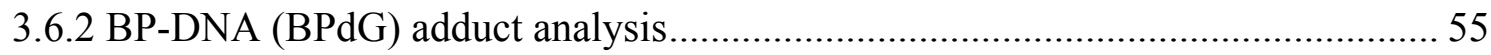

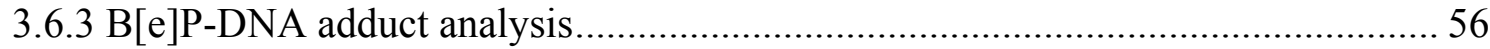

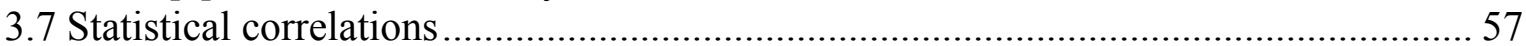

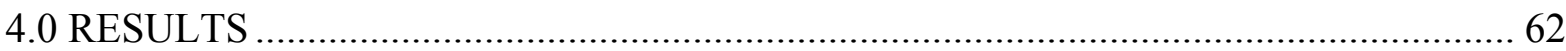

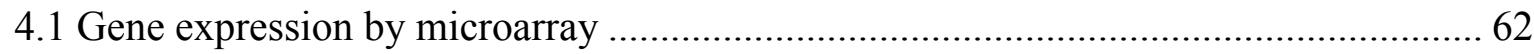

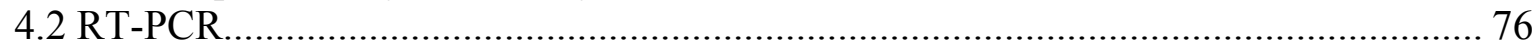

4.2.1 Confirmation of gene expression on microarrays ............................................... 76

4.2.2 CYP1A1 and CYP1B1 expression in 20 NHMECs on treatment with BP in the

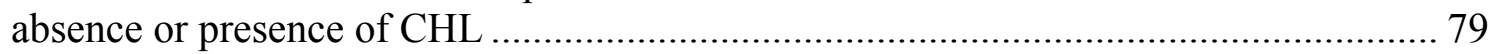
4.2.3 CYP1A1 and CYP1B1 expression in 2 NHMECs on treatment with $\mathrm{BP}$ or $\mathrm{B}[\mathrm{e}] \mathrm{P}$ in

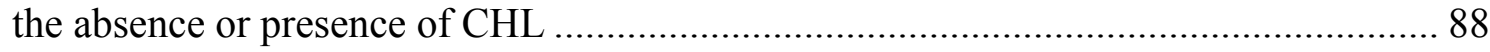
4.2.4 CYP1A1 and CYP1B1 expression in MCF-7 and M00012 on treatment with BP in

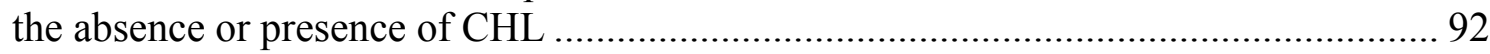
4.2.5 CYP1A1 and CYP1B1 expression in MCF-7 and M00012 on treatment with BP in the absence or presence of different CHL concentrations ............................................ 96

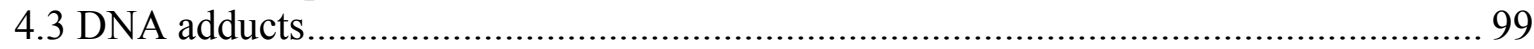
4.3.1 BP-DNA adducts in 20 NHMECs on treatment with BP in the absence or presence

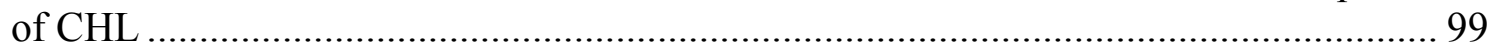
4.3.2 BP-DNA and B[e]P-DNA adducts in 2 NHMECs on treatment with $\mathrm{BP}$ or $\mathrm{B}[\mathrm{e}] \mathrm{P}$ in the absence or presence of CHL ...................................................................... 101 4.3.3 BP-DNA adducts in MCF-7 and M00012 or 20 NHMECs on treatment with BP in the absence or presence of CHL .............................................................................. 103 4.3.4 BP-DNA adducts in MCF-7 and M00012 on treatment with BP in the absence or presence of different CHL concentrations .............................................................. 105

4.4 Correlation of CYPI gene expression to BP-DNA adducts....................................... 107 4.4.1 Correlation of cell strain origin with CYPI gene expression and DNA adduct

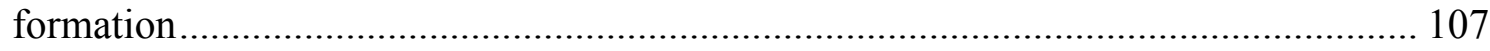
4.4.2 Correlation of CYP1 expression to DNA adducts in 20 NHMECs on treatment with BP in the absence or presence of CHL ............................................................. 107 4.4.3 Correlation of CYP1 expression to DNA adducts in 2 NHMECs on treatment with $\mathrm{BP}$ or $\mathrm{B}[\mathrm{e}] \mathrm{P}$ in the absence or presence of CHL ................................................... 108 4.4.4 Correlation of CYP1 gene expression to DNA adducts in MCF-7 and M00012 or 20 NHMECs on treatment with BP in the absence or presence of CHL ..................... 109 4.4.5 Correlation of CYP1 gene expression to DNA adducts in MCF-7 and M00012 on treatment with BP in the absence or presence of different CHL concentrations .......... 109

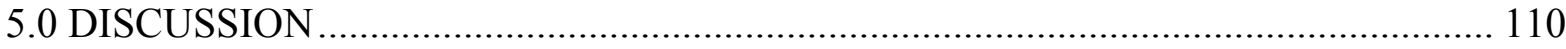

5.1 Gene expression by microarrays ......................................................................... 110

5.1.1 Genes altered by BP treatment.......................................................................... 110 
5.1.2 Genes altered by Pre CHL + co-treatment

5.2 Correlation of gene expression on microarrays versus RT-PCR ............................... 117

5.3 Genetic damage in terms of CYPI induction and DNA adduct formation in 20

NHMECs on treatment with BP in the absence or presence of CHL .............................. 118

5.4 Genetic damage in terms of CYP1 induction and DNA adduct formation in 2 NHMECs

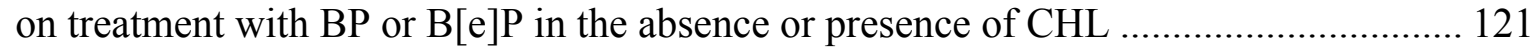

5.5 Genetic damage in terms of CYP1 induction and DNA adduct formation in MCF-7 cells versus an NHMEC (M00012) on treatment with BP or B[e]P in the absence or presence of CHL 123

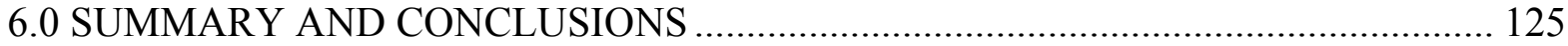

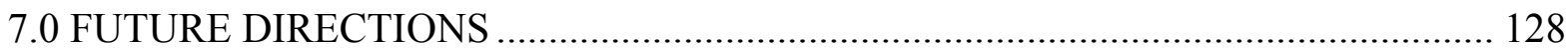

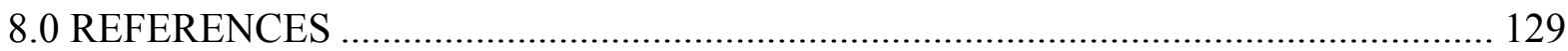

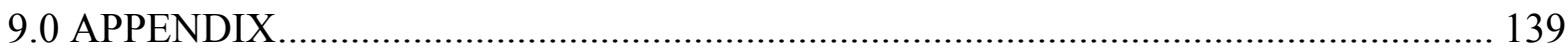




\section{LIST OF FIGURES}

Figure 1(A) Benzo(a)pyrene $\quad 9$

$\begin{array}{lll}\text { Figure 1(B) } & \text { Benzo(e)pyrene }\end{array}$

$\begin{array}{lll}\text { Figure 2 } & \text { Metabolic pathways of BP }\end{array}$

$\begin{array}{lll}\text { Figure 3 } & \text { Metabolic activation of BP } & 17\end{array}$

Figure 4 Structures of some of the biologically important metalloporphyrins 26

Figure 5 Multiple levels in the regulation of P450s 32

Figure 6 Representative scanned image obtained following scanning of arrays $\mathbf{5 0}$

Figure 7 Fold change scatter plots of Hu-Gene133A arrays $\quad 63$

Figure 8 Graphical display of all genes altered by SLR $\geq 1.5$ on Hu-Gene 66 $133 \mathrm{~A}$ arrays

$\begin{array}{lll}\text { Figure 9 } & \text { Correlation coefficient gene-expression clusters } & 67\end{array}$

Figure 10 Graphical representation of all immune response genes altered by $\mathbf{7 0}$ SLR $\geq 1.5$ on Hu-Gene $133 \mathrm{~A}$ arrays

Figure 11 Cross-talks between immune response genes altered by BP treatment

Figure 12 Cross-talks between immune response genes altered by Pre CHL $+\quad \mathbf{7 3}$ co-treatment

Figure 13 Correlation of $C Y P 1 A 1$ expression to that of $C Y P 1 B 1$ in the 20 NHMECs

Figure 14 Correlation between the percentage modulation in the expression of $\mathbf{8 5}$ $C Y P 1 A 1$ to that of $C Y P 1 B 1$

Figure 15 Correlation of $C Y P 1 A 1$ expression in the absence and presence of $\mathbf{8 6}$ CHL

Figure 16 Correlation of $C Y P 1 B 1$ expression in the absence and presence of $\mathrm{CHL}$

Figure 17 Comparison of CYP1Al expression by $\mathrm{BP}$ or $\mathrm{B}[\mathrm{e}] \mathrm{P} \pm \mathrm{CHL}$ 
Figure 18 Comparison of $C Y P 1 B 1$ expression by $\mathrm{BP}$ or $\mathrm{B}[\mathrm{e}] \mathrm{P} \pm \mathrm{CHL}$

Figure 19 CYPIA1 expression patterns on treatment of MCF-7 cells or $\mathrm{M} 00012$ (a NHMEC) with $\mathrm{BP} \pm \mathrm{CHL}$

Figure 20 $C Y P 1 B 1$ expression patterns on treatment of MCF-7 cells or $\mathrm{M} 00012$ (a NHMEC) with $\mathrm{BP} \pm \mathrm{CHL}$

Figure 21 CYP1A1 expression patterns in MCF-7 and M00012 by BP in the absence or presence of a range of CHL concentrations

Figure 22 $C Y P 1 B 1$ expression patterns in MCF-7 and M00012 by BP in the absence or presence of a range of CHL concentrations

Figure 23 Synchronous fluorescence spectra obtained from standard benzo(a)pyrene-r-7,t-8,c-9,t-10-tetrahydrotetrol

Figure 24 Synchronous spectra of the B[e]P treated cells

Figure 25 BP-DNA adduct levels $/ 10^{8}$ nucleotides in MCF-7 and M00012 on treatment with $\mathrm{BP} \pm \mathrm{CHL}$

Figure 26 BP-DNA adduct levels $/ 10^{8}$ nucleotides in MCF-7 and M00012 on treatment with BP in the absence or presence of a range of $\mathrm{CHL}$ concentrations 


\section{LIST OF TABLES}

Table 1 List of receptors and factors associated with the induction of some

P450s involved with endogenous substrate metabolism

Table 2 Details of different experiments carried out in the present study

Table 3 NHMECs and MCF-7 cells treatment protocol

Table 4 Treatment protocol for the comparison of BP and B[e]P across 2 NHMECs (M98015 and M98025)

Table 5 Treatment protocol for the CHL dose response study comparing MCF-7 and a NHMEC (M00012) across a range of CHL concentrations

Table 6 Table representing the number and characteristics of the probe sets used to generate the clusters displayed in Figure 9

Table 7 Pathways in which immune response (IR) genes altered by SLR $\geq 1.5$ on Hu-Gene133A arrays on comparison of BP treated cells versus control vehicle are involved

Table 8 Pathways in which immune response (IR) genes altered by SLR $\geq 1.5$ on Hu-Gene133A arrays on comparison of CHL treated cells (pre CHL + co-treated) versus control vehicle are involved

Table 9(A) Correlation of fold change (FC) on Hu-Gene 133A arrays/gene-chip least one of the 6 NHMECs when treated with BP alone 
Table 9(B) Correlation of fold change (FC) on Hu-Gene 133A arrays/gene-chip

(GC) and RT-PCR for a partial list of genes altered by SLR $\geq 1.5$ in at least one of the 6 NHMECs when treated with pre-CHL followed by co-treatment

Table 10 Correlation of FC on gene-chip (GC) versus RT-PCR for a selected $\mathbf{7 8}$ set of immune response genes altered by SLR $\geq 1.5$ in at least one of the 6 NHMECs on treatment with BP in the absence or presence of $\mathrm{CHL}$

Table 11 Inter-individual variations in the induction and modulation of CYP1A1 upon exposure of NHMECs to either BP alone or in combination with CHL

Table 12 Inter-individual variations in the induction and modulation of CYP1B1 upon exposure of NHMECs to either BP alone or in combination with $\mathrm{CHL}$

Table 13 Basal $C Y P 1 A 1$ and $C Y P 1 B 1$ expression levels $(\triangle \mathrm{Ct})$ in the 20 NHMECs normalized to that of GAPDH

Table 14 Inter-individual variations in BP-DNA adduct formation on exposure of NHMECs to either BP alone or in combination with CHL 


\section{LIST OF ABBREVIATIONS}

\begin{tabular}{|c|c|}
\hline $4-\mathrm{OHE}_{2}$ & 4 hydroxy estradiol \\
\hline AhR & Aryl hydrocarbon receptor \\
\hline ARNT & AhR nuclear translocator \\
\hline ATCC & American Type Culture Collection \\
\hline $\mathrm{B}[\mathrm{e}] \mathrm{P}$ & Benzo(e)pyrene \\
\hline $\mathrm{BP}$ & Benzo(a)pyrene \\
\hline \multirow[t]{2}{*}{ BPDE } & $( \pm)-7 \beta, 8 \alpha$, dihydroxy $9 \alpha, 10 \alpha$-epoxy- $7,8,9,10-$ \\
\hline & tetrahydrobenzo $[\alpha]$ pyrene \\
\hline \multirow[t]{2}{*}{ BPdG } & (7R)-N2-(10[7b,8a, 9a-trihydroxy-7,8,9,10-tetrahydro- \\
\hline & benzo[a]pyrene]yl)-deoxyguanosine \\
\hline BSA & Bovine serum albumin \\
\hline cDNA & Copy DNA \\
\hline $\mathrm{CHL}$ & Chlorophyllin \\
\hline cRNA & Complementary RNA \\
\hline CYP1A1 & Cytochrome P4501A1 \\
\hline CYP1B1 & Cytochrome P4501B1 \\
\hline CYP3A4 & Cytochrome P4503A4 \\
\hline DMSO & Dimethyl sulfoxide \\
\hline DMT & Data Mining Tool \\
\hline DNA & Deoxyribose nucleic acid \\
\hline $\mathrm{FC}$ & Fold change \\
\hline GC & Gene chip \\
\hline $\mathrm{HCC}$ & Hepatocarcinoma \\
\hline $\mathrm{HH}$ & Halogenated hydrocarbon \\
\hline IARC & International Agency for Research on Cancer \\
\hline MAS & Microarray Suite \\
\hline NHMEC & Normal human mammary epithelial cell \\
\hline
\end{tabular}




$\begin{array}{ll}\text { PAH } & \text { Polycyclic aromatic hydrocarbon } \\ \text { PBST } & \text { phosphate buffered saline containing } 0.05 \% \text { Tween-20 } \\ \text { PKA } & \text { protein kinase A } \\ \text { RNA } & \text { Ribose nucleic acid } \\ \text { RT-PCR } & \text { Real time polymerase chain reaction } \\ \text { SAPE } & \text { streptavidin phycoerythroprotein } \\ \text { SC } & \text { Solvent control/control vehicle } \\ \text { SFS } & \text { synchronous fluorescence spectrometry } \\ \text { SLR } & \text { Signal log ratio }\end{array}$




\subsection{INTRODUCTION}

Human exposures to various compounds such as polycyclic aromatic hydrocarbons (PAHs), halogenated hydrocarbons (HHs), aromatic amines (AAs), microbial toxins, drugs and other xenobiotic compounds occur through diet, occupation, medicines and/or environment. Benzo(a)pyrene (BP) is a PAH found in tobacco smoke, vehicle exhaust, indoor heating and various industrial processes, and it is a ubiquitous environmental pollutant and potent procarcinogen (IARC 1987; Osborne et al. 1987). On entering the body it can follow a number of routes some of which lead to its bioactivation or detoxication.

Metabolic activation of PAHs (e.g., BP) is necessary for them to exert their adverse biological and carcinogenic effects, and cytochrome P450 metabolism is a key factor in this process. Cytochrome P450s (CYPs) constitute a superfamily of heme thiolate proteins, that are likely the most important classes of enzymes involved in the metabolism of all xenobiotics (Guengerich 2001). Through their action, together with other enzymes (epoxide hydrolases) the bioactivation of BP proceeds, leading to the generation of highly reactive electrophiles capable of damaging cellular macromolecules such as DNA, RNA and protein (Weston et al. 2005). In addition, CYP450s have a normal role in hormone metabolism, and since $\mathrm{BP}$ acts to generally induce the activity of a range of CYP450s through activation of the aryl hydrocarbon receptor, BP and other PAHs may be a significant factor in hormonal carcinogenesis

$\mathrm{BP}$, by acting as a ligand for the AhR, enters the cell and induces CYP genes (OeschBartlomowicz et al. 2005). CYP1A1, CYP1B1 and CYP3A4 are the predominant CYP isoforms documented to be involved in the metabolism of BP. The predominant pathway of 
metabolism involves the initial oxidation of BP by CYP1A1/CYP1B1 at the 7, 8 position followed by hydrolysis by epoxide hydrolase to dihydrodiols and then further oxidation at the 9, 10 olefinic bond to yield dihydrodiol epoxides, which being highly electrophilic bind covalently to DNA leading to genetic damage (Weston et al. 2005). CYP1A1 and CYP1B1 are also potent steroid hydroxylases with CYP1B1 being a potent estradiol 4 hydroxylase. 4$\mathrm{OHE}_{2}$ has been shown to possess a strong cell proliferative effect in various estrogen responsive tissues such as those of the breast and the endometrium and is thereby implicated in their carcinogenesis (Cavalieri et al. 2002).

Given the ubiquitous nature of BP, a complete avoidance of exposure may be difficult. Chemopreventive agents that block or even reverse the carcinogenic process may therefore be a useful alternative consideration. CHL, a water soluble sodium-copper or potassium-copper metalloporphyrin has been shown to exhibit anti-carcinogenic, antimutagenic, anti-tumorigenic and anti-oxidant properties in various studies and model systems (Sarkar et al. 1994; Tachino et al. 1994; Dashwood 1997a; Reddy et al. 1999; Kamat et al. 2000). It has also been used as a safe and effective intervention strategy in various developing parts of Africa and China with populations at high risk for developing hepatocarcinoma (HCC) due to the regular consumption of aflatoxin contaminated foods (Kensler et al. 2004).

Due to its carcinogenic properties, BP has been a compound of intense research interest since its isolation in the 1930s. However, very few studies have been conducted on the effect of BP on genome wide gene expression patterns, effect of intervention agents on modulating these expression patterns on a genomic scale and in turn the effects of this modulation to the individual in question. In addition, malignantly transformed cells are often 
used as alternatives to normal cells in in vitro studies due to their ease in maintenance and the assumption of their similarity to normal cells in response to carcinogenic stress. However, whether or not malignantly transformed cells are appropriate models to use in studies of metabolic activation and chemical carcinogenesis is an important question to consider. This dissertation considers the metabolism and activation of an important, carcinogenic polycyclic aromatic hydrocarbon to which everyone is exposed. It further considers inter-individual variation in certain metabolic factors that impact an individual's risk of cancer, and it examines the extent to which these factors may be mitigated by the introduction of a chemical intervention. 


\section{Objectives}

The goals and objectives of these studies were to:

- Assess genome-wide changes in gene expression patterns among a panel of 6 normal human mammary epithelial cells (NHMECs) on exposure to BP in the absence or presence of CHL in order to seek biomarkers for chemopreventive intervention.

- $\quad$ Determine the extent of inter-individual variation in CYP1A1 and CYP1B1 gene expression among a larger panel of 20 NHMECs on exposure to BP in the absence or presence of CHL.

- Determine the extent of inter-individual variation in BP-DNA adduct formation among a larger panel of 20 NHMECs on exposure to BP in the absence or presence of CHL.

- $\quad$ Examine BP and B[e]P in parallel for their effect on CYP1A1 and CYP1B1 gene expression and DNA adduct formation in 2 NHMECs in the absence or presence of CHL.

- Examine NHMEC(s) in parallel with a cancerous cell line, MCF-7 for their effect on CYP1A1 and CYP1B1 gene expression and DNA adduct formation on exposure to BP in the absence or presence of CHL. 


\subsection{REVIEW OF LITERATURE}

\subsection{Introduction}

Cancer is the second largest cause of mortality next only to cardiovascular diseases. Nearly 1.4 million new cancer cases (excluding noninvasive cancer) are expected to be diagnosed in the US in 2006 and more than 500,000 will die (http://www.cancer.org). A large proportion of these cancer deaths are attributable to nutrition, lifestyles and occupational exposures to many carcinogens.

Cancers arise in epithelial tissues (carcinomas), mesenchymal tissues (sarcomas), haemato-lymphoid system (leukemias and lymphomas) and other tissues of the body (Knowles et al. 2005). These cancers are both benign and malignant.

Human exposures to a range of chemicals and toxins many of which may be potentially carcinogenic occur through air, water, diet, lifestyle and/or occupation. Biotransformation and metabolism of many of these chemicals are the primary cause of toxic manifestations. Cytochrome P450s (CYPs) form the major superfamily of enzymes involved in many of these Phase I metabolic reactions in addition to their roles in steroid genesis and drug metabolism. Given the widespread distribution and potentially multiple routes of exposure of many of these chemicals alternative strategies involving the use of chemopreventive measures to block or even reverse some of the genetic damage induced by these agents may be a viable approach. 


\subsection{Chemical carcinogenesis}

Chemical carcinogenesis typically involves a complex series of events starting with the bioactivation of the chemicals to DNA damaging metabolites followed by damage of cellular macromolecules and mutation fixation. Reports on increased incidences of nasal polyps among dippers and enhanced rates of scrotal cancer in English chimney sweeps were some of the early documented cases of chemical carcinogenesis (Poirier 2004).

Early chemical carcinogenesis studies in the 1930s with attempts to isolate some of the carcinogenic constituents of coal tar has progressed rapidly ever since (Phillips 1983). An important landmark was the understanding that binding of reactive electrophiles (in case of genotoxic agents) to cellular macromolecules being associated with enhanced risk of carcinogenesis. However, these early studies believed the interaction to occur with proteins rather than DNA (Miller 1951). The work by Brookes and Lawley served to be some of the seminal work which demonstrated the binding of PAHs to DNA and its correlation to carcinogenicity (Brookes et al. 1964). Subsequently, it has also become known that free radicals that may be generated by CYP mediated catalytic cycling of xenobiotics could also potentially cause free radical damage of cellular macromolecules. Non-genotoxic agents may cause damage by oxyradical formation, depurination, toxic cell death or even by modifying the effects of genotoxic agents (Yuspa et al. 1997). All these can be involved in the multistage process of chemical carcinogenesis that can be conceptually divided into 4 component process: tumor initiation, tumor promotion, malignant conversion and tumor progression. 
Tumor initiation is a rare event the frequency of which is dependent on the carcinogen dosage. It may involve DNA adduct formation (as in case of genotoxic agents) leading to the inactivation of tumor suppressor genes and/or activation of protooncogenes or events such as methylation of the promoters of tumor suppressor genes (as in case of non-genotoxic agents) leading to their silencing. This requires the changes to occur in a population of dividing cells so as to 'fix' the mutations. In general a strong correlation has been observed between the extent of DNA adduct formation and tumor formation.

Tumor promotion involves a clonal expansion of the initiated cells. Tumor promoters are agents that are generally non-mutagenic and non-carcinogenic by themselves but which enhance the carcinogenicity of tumor initiators. These usually possess multiple mechanisms of action and generally do not require any metabolic activation for the activity. They mainly serve to reduce the latency period for tumor formation when dosed in conjunction with the initiator. Tumor promoters generally bring about their effects through activation of cell surface receptors, stimulation of cell proliferation, perturbation of signal transduction pathways, inhibition of apoptosis or by modulation of cytosolic and nuclear factors. Agents capable of functioning both as a tumor initiator and promoter are regarded as complete carcinogens e.g.: 4-amino biphenyl. Tumor promotion is considered the chief rate limiting step in experimental carcinogenesis.

Malignant conversion involves accumulation of further genetic changes leading to the transformation of preneoplastic cells to express a malignant phenotype. This often involves multi-focal changes in premalignant lesions such as up-regulation of AP-1, gene 
amplification, exposure of cells to DNA damaging agents and over-expression of cell cycle regulatory proteases. The changes occurring may be reversible where regression of lesions occur when the promoting agent is removed prior to malignant conversion.

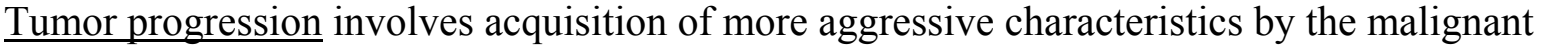
cells facilitating their spread and invasion beyond the primary site and often involves events such as complex genetic alterations (translocations, deletions, gene amplifications, duplications), ectopic changes in hormone production and activation of protooncogenes (through point mutations or gene amplifications) and inactivation of tumor suppressor genes.

\subsection{Chemical classes}

\subsubsection{Polycyclic aromatic hydrocarbons (PAHs)}

PAHs are a class of more than 100 known compounds that are composed of variable numbers of fused benzene rings. They are ubiquitous environmental pollutants formed from the incomplete combustion of organic matter and are also generated as by-products from various industrial processes. Pure PAHs are usually colorless, white or pale yellow solids, generally odorless, but in some cases have a mild faint odor. These compounds almost most occur as complex chemical mixtures in nature.

Considerable interest in PAHs stems from the ability of many of them to cause toxicity and cancers of various organs (Ramesh et al. 2004). PAHs are chemically inert, and they require metabolic activation to exert their biological effects (Weston et al. 2005). Benzopyrenes, are members of the PAH family of compounds. Two of these benzo(a)pyrene (BP) and benzo(e)pyrene $(\mathrm{B}[\mathrm{e}] \mathrm{P})$ were used in various experiments in this study. 
Benzopyrenes

Benzopyrenes, prototypical carcinogens found generally with various other PAHs in tobacco smoke, charbroiled foods and in the combustion products of organic matter and various organic fuels are ubiquitous environmental pollutants (Hooven et al. 2005). BP, a highly carcinogenic $\mathrm{PAH}$, has been a compound of extensive research interest since its first isolation by Kennaway et. al., in 1930s from 2 tons of coal tar pitch (Kennaway 1955). According to the International Agency for Research on Cancer (IARC) it has been placed under Group 2A (probably carcinogenic to humans) (IARC 1987). The structure of BP is shown in Figure 1(A).

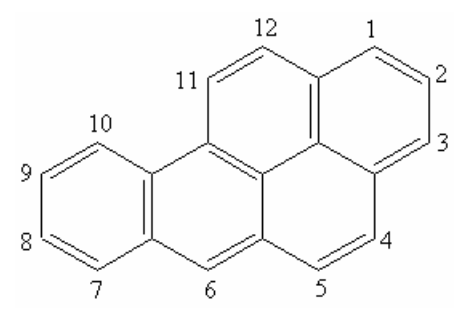

Figure 1: (A) Benzo[a]pyrene (BP)

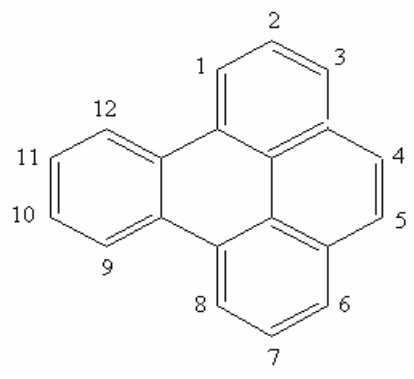

(B) Benzo[e]pyrene (B[e]P)

$\mathrm{B}[\mathrm{e}] \mathrm{P}$ is a structural isomer of $\mathrm{BP}$. The low carcinogenicity of $\mathrm{B}[\mathrm{e}] \mathrm{P}$ in contrast to BP has caused very few studies to be carried out on $\mathrm{B}[\mathrm{e}] \mathrm{P}$ compared to $\mathrm{BP} . \mathrm{B}[\mathrm{e}] \mathrm{P}$ is only weakly mutagenic and carcinogenic compared to BP and has been assigned IARC rank of Group 3 (not classifiable as to the carcinogenicity to humans) (IARC 1987). The structure of $\mathrm{B}[\mathrm{e}] \mathrm{P}$ is presented in Figure 1(B). 
However, because it is found with BP and other highly carcinogenic hydrocarbons in the environment, its effect on modulating the carcinogenicity of these hydrocarbons is to be addressed.

\subsubsection{Aromatic amines and heterocyclic amines}

Aromatic amines are carcinogens present in tobacco smoke, diesel exhaust and some cooked foods. They consist of one or more rings of unsaturated or cyclic hydrocarbons along with nitrate $\left(\mathrm{NH}_{2}\right)$ groups. They are implicated in cancers of the bladder, colon and ureter (Weston et al. 2005).

Heterocyclic amines are formed from high temperature cooking of foods $\left(>150^{\circ} \mathrm{C}\right)$ mainly from pyrolysis of the amino acids, creatine and creatnine and sugars such as glucose. These substances have been found to be potent mutagens in Ames Salmonella assay and are carcinogens in laboratory animals (Layton et al. 1995). These have also been found to form liver tumors in primates (Adamson 1989).

\subsubsection{Aflatoxins}

These are chemicals which represent a family of structurally related difuranocoumarin derivatives produced by certain fungi (Aspergillus flavus and $A$. parasiticus) that contaminate peanuts, soybean and certain cereals. The production of theses toxins are affected by environmental and strain specific factors (Sudakin 2003). Aflatoxin B1 and G1 are more mutagenic and carcinogenic than aflatoxin B2 and G2 (Weston et al. 2005). Exposure to aflatoxin B1 has been shown to be linked to enhanced risk of liver cancer in 
human populations exposed to dietary aflatoxins in certain parts of China and Africa (Kensler et al. 2004).

\subsubsection{N-nitrosamines}

These are ubiquitous environmental pollutants found in certain foods such as cured meats, alcoholic beverages, certain cosmetics, oils and tobacco. N-nitrosamines can also be formed endogenously through nitrosation where an amine reacts with nitrate alone or in the presence of acid (Weston et al. 2005). Exposures to dietary nitrosamines have been associated with risk of esophageal and gastrointestinal cancers while exposure to tobacco specific nitrosamines such as 4-(methylnitrosoamino)-1-(3-pyridyl)-1-butanone have been associated with enhanced risk of lung cancer (Goldman et al. 2003).

\subsubsection{Non-genotoxic agents}

These are mainly agents which tend to bioaccumulate in biological systems (e.g.: pesticides, herbicides) and cause disease upon prolonged exposure. There is no conclusive evidence of their role in causing cancers but they may modify the effects of some of genotoxic carcinogens. In other cases they may manifest their effects by causing toxic cell death, oxyradical formation, disruption of hormonal balance, dysregulation of growth factors and by disruption of certain signaling pathways (Yuspa et al. 1997; Weston et al. 2005). 


\subsection{Metabolism of carcinogens}

Once internalized most chemical carcinogens are subject to competing processes of metabolic activation (Phase I reactions) versus detoxication (Phase II reactions). The metabolic route of some of the chemical classes listed above is discussed below.

\subsubsection{PAHs}

\section{Benzopyrenes}

$\mathrm{BP}$ is one of the best characterized PAHs whose metabolism has been studied extensively. It acts as a ligand for Ahr, a basic helix-loop-helix (bHLH)/Per-ARNT-Sim (PAS) homology domain family of transcription factors. Various other planar aromatic compounds and many of their halogenated derivatives also act as Ahr ligands. BP has been found to be a strong Ahr ligand while B[e]P a weak Ahr ligand (Sterling et al. 1994). In vertebrates, Ahr exists in the cytosol complexed with a dimer of Hsp90 and several Hsp90 accessory proteins (Puga et al. 2005). Ahr ligands following entry of the cell by diffusion, bind Hsp90 associated Ahr and subsequently cause a conformational change in Ahr leading to its increased affinity for DNA. The conformation change causes liganded Ahr to translocate to the nucleus, exchange Hsp90 for Arnt (aryl hydrocarbon nuclear translocator), another bHLH/PAS transcription factor ultimately leading to the formation of an Ahr, Arnt and ligand complex. This complex in turn binds to xenobiotic response elements (XRE) in the upstream regions of CYP genes leading to their enhanced transcription (Schmidt et al. 1996; Vrzal et al. 2004). In some rare cases unliganded Ahr has been found to undergo nuclear translocation and it is suggested that a universal secondary mediator like cAMP may play a role in this mechanism (Oesch-Bartlomowicz et al. 2005). 
Many studies on the mechanism of BP metabolism occurred during the period between 1950 -1990. The metabolism of BP generally proceeds in 2 phases; Phase I involves the conversion of BP to oxidized derivatives (epoxides, phenols, diols and quinines) and Phase II reactions involve the conversion of these derivatives to polar metabolites by conjugation with sulfate, glucuronic acid or glutathione (Osborne et al. 1987).

The primary metabolites are three epoxides: 4,5-epoxide, 7,8-epoxide, and 9,10epoxide while 1,2-epoxide and 2,3-epoxide are theoretical intermediates that are nonenzymatically converted to phenols. 4, 5-epoxide is the most stable of the epoxides that can be isolated. These products of initial oxidation are generated by the action of various CYPs principally CYP1A1, CYP1B1 and CYP3A4. The epoxides can subsequently be hydrolyzed to the corresponding trans-diols/dihydrodiols (t-4,5 diol, t-7,8 diol or t-9,10 diol) by the action of epoxide hydratase $(\mathrm{EH})$ or be converted non-enzymatically to phenols or undergo conjugation by glutathione S-transferases to form the corresponding glutathione conjugates (Gelboin 1980). Diols formed can exist in either the cis or trans configuration. All diols formed from PAHs other than BP in the presence of mammalian microsomal enzymes exist in the trans configuration. However, all diols formed enzymatically from BP are trans and (-) enantiomers of high optical purity (Gelboin 1980).

1-OH, 3-OH, 6-OH, 7-OH and 9-OH are the 5 major phenol metabolites (with the 3$\mathrm{OH}, 7-\mathrm{OH}$ and 9-OH metabolites being the most common) which can subsequently be converted to quinones/diones (1,3 quinone, 3,6 quinone and 6,12 quinone) or be conjugated to glucuronic acid by UDP glucuronsyl transferases (encoded by 2 gene families, UGT1 and UGT2 with several sub-families and members) to yield glucuronide conjugates. The glucuronide conjugates may be acted upon by $\beta$-glucuronidase present in the intestinal 
microflora to release the parent compound, which, being electrophilic may attack DNA and other cellular macromolecules (Parkinson 2003). The quinones formed from the phenols may be conjugated to sulfates by sulfotransferases (encoded by 5 gene families, SULT1-SULT5 with several subfamilies) and subsequently be converted to the corresponding sulfate metabolites or be conjugated to glutathione to produce glutathione conjugates (Guillen et al. 2003). The phenols may also be directly converted to sulfate metabolites by the action of sulfotransferases. The formation of phenols, sulfates, glutathione and glucuronide conjugates are all considered to be more of detoxification pathways enhancing the hydrophilicity of the compounds and therefore aiding in their excretion from the system. Dihydrodiol metabolites can undergo further oxidation by CYPs (mainly CYP1B1 and CYP3A4) to produce dihydrodiol epoxides (Gelboin 1980) or may undergo conversion to more polar phenol diols (6-OH-7,8 diol, 1-OH-9,10 diol, 3-OH-9,10 diol, 9-OH-4,5 diol).

The two common stereoisomeric dihydrodiol epoxides (of 4 possible forms) are r-7,t8 diol c-9,10 hydroxy BP/ $( \pm) 7 \alpha, 8 \beta$-dihydroxy-9 $\alpha, 10 \alpha$-epoxy-7,8,9,10 tetrahydroBP (BPDE1/syn isomer/cis isomer) and r-7,t-8 diol t-9,10 dihydroxy $\mathrm{BP} /( \pm) 7 \alpha, 8 \beta$-dihydroxy9 $\beta, 10 \beta$-epoxy-7,8,9,10 tetrahydroBP (BPDE2/anti isomer/trans isomer) (Kim et al. 1998; Rubin 2001). The preferred oxygenation site and subsequently the preferred dihydrodiol epoxide formed appears to depend on the CYP isoform catalyzing the process and hence is a function of the tissue or enzyme preparation used (Gelboin 1980). Both BPDE1 and BPDE2 are reactive capable of binding to DNA, RNA and proteins forming bulky covalent addition compounds called adducts. The first experimental evidence that the anti isomer of BPDE may be the ultimate DNA binding metabolite was from work of Sims et. al. (Sims et al. 1974). Both, BPDE1 and BPDE2 form a C-10 carbonium ion intermediate which is 
responsible for their reactivity towards cellular nucleophiles such as DNA and proteins. Among the two stereoisomers BPDE2 has been found to be even more reactive than BPDE1 and hence has been studied extensively (Kim et al. 1998). Both diol epoxides however are unstable in aqueous media and are hydrolyzed spontaneously to tetrols. BPDE1 can be hydrolyzed to 2 tetrols, ( $( \pm)$ BP r-7,t-8 c-9,c-10 tetrahydrotetrol (RTCC), the major tetrol and $( \pm)$ BP r-7,t-8 c-9,t-10 tetrahydrotetrol (RTCT), the minor tetrol while BPDE2 can also be hydrolyzed to 2 tetrols, $( \pm)$ BP r-7,t-8 t-9,c-10 tetrahydrotetrol (RTTC), the major tetrol and $( \pm)$ BP r-7,t-8 t-9,t-10 tetrahydrotetrol (RTTT), the minor tetrol. [The R, T, C system of nomenclature was suggested by the American Chemical Society where $r=$ relative, the position to which all other positions relate, $\mathrm{t}=$ trans and $\mathrm{c}=\mathrm{cis}]$. Both BPDE1 and BPDE2 can be reduced in the presence of NADH or NADPH to the corresponding triols. This reduction occurs non-enzymatically and is dependent on the tissue concentration of NADH or NADPH. The triols and tetrols are polar and undergo elimination through excretion. A summary of these various routes during the metabolism of BP is presented in Figure 2. A more detailed representation of the pathway leading to metabolic activation and the formation of adducts is presented in Figure 3.

Metabolically activated BP (BPDE) binds DNA, RNA and proteins. The binding to proteins may be 10 times greater than to nucleic acids. Apart from BPDE, the oxides as well as diols are also known bind to proteins and nucleic acids (Osborne et al. 1987). Electron rich amino acids such as tyrosine, histidine and tryptophan may play a vital role in protein binding. When binding to DNA, deoxyguanosine appears to be the preferred base though adducts with all other bases of DNA are also known (Dipple et al. 1984). Some studies have also found modification of the phosphate backbone of nucleic acids. In case of binding to 
RNA there seems to be some interaction of the diol epoxides with uracil residues (Gelboin 1980). In case of guanine the major site of modification is the exocyclic amino group at position 2 though a minor product may also be formed at N-7. Adducts are also possible with the exocyclic amino group at position 6 of deoxyadenosine and the amino group of deoxycytidine (Dipple et al. 1984). The diol epoxide seems to be the predominant adduct involved in DNA binding.

Given the lower carcinogenicity of $\mathrm{B}[\mathrm{e}] \mathrm{P}$ compared to $\mathrm{BP}$ relatively fewer studies have been carried out on this compound. Metabolism of $\mathrm{B}[\mathrm{e}] \mathrm{P}$ has been found to yield $\mathrm{B}[\mathrm{e}] \mathrm{P}-$ 4,5-dihydrodiol, 1-hydroxy-BeP and 3-hydroxy-BeP along with small amounts of B[e]P9,10-dihydrodiol and a few other secondary metabolites. Of these the 4,5 dihydrodiol appears to be the major metabolite. Other studies have also found an unidentified phenol (probably 1hydroxy-BeP), 3-hydroxy-BeP and a quinone as metabolites but their proportions are much lower than $\mathrm{B}[\mathrm{e}] \mathrm{P}-4$, 5-dihydrodiol. However, $\mathrm{B}[\mathrm{e}] \mathrm{P}-9,10$-dihydrodiol is the only derivative whose metabolism has been studied. On incubation of this derivative with liver microsomal about $13 \%$ was converted to 9,10-epoxy-11,12-dihydroxy-9,10,11,12-teterahydro-BeP while the rest was recovered as $\mathrm{B}[\mathrm{e}] \mathrm{P}-4,5,9,10$-tetrahydrotetrol and 2 triols. 9,10-epoxy-11,12dihydroxy-9,10,11,12-teterahydro-B[e]P reacts directly with DNA, the principal adducts having $\mathrm{C} 9$ of guanine attached to exocyclic $\mathrm{N} 2$ of guanine. A few other minor adducts including ones with deoxyadenosine have also been found (Osborne et al. 1987). 


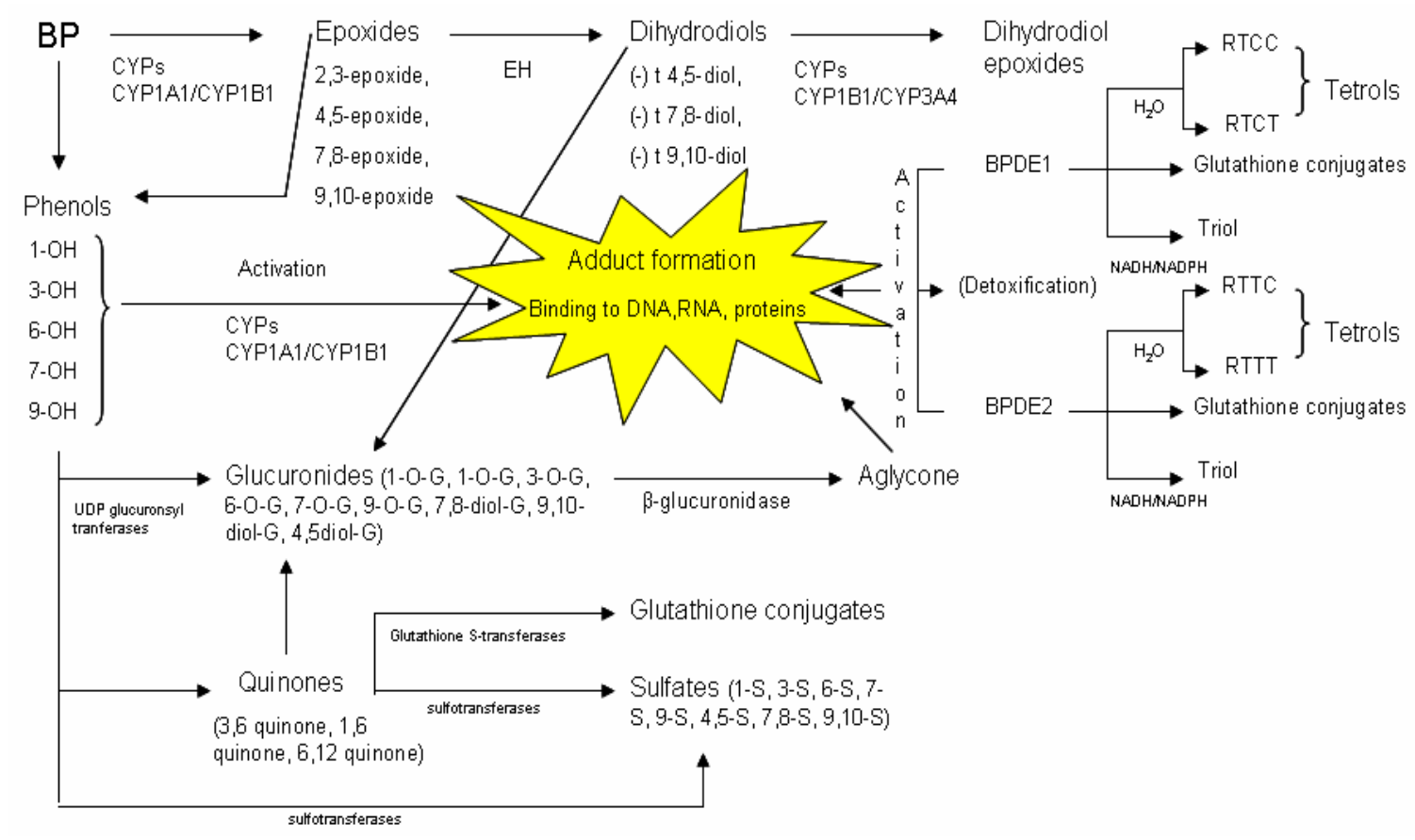

Figure2: Metabolic pathways of BP. The different routes of metabolism listed here are however not exhaustive.

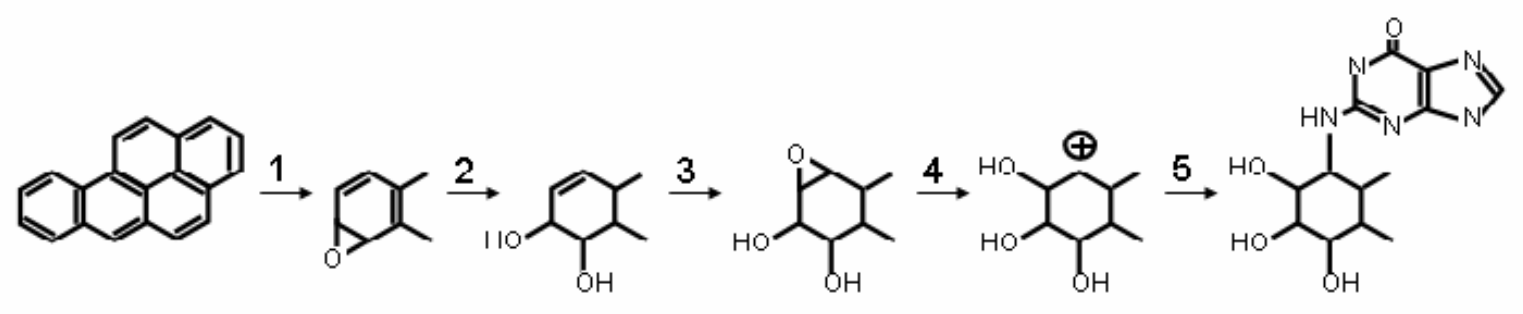

Figure 3: The metabolic activation of BP has been widely studied. (1) CYP1A1 and CYP1B1 catalyze the conversion of the parent compound to arene oxides. (2) Arene oxides are converted to dihydrodiols by EH (3) The resulting dihydrodiols are further oxidized at the site of the olefinic bond (4) Vicinal diol epoxides are highly unstable and undergo spontaneous arene ring opening to yield carbocation. (5) The electrophilic carbocation can form a covalent bond with exocyclic amino group of deoxyguanosine. The resulting adducts resides in the minor groove of DNA. 


\subsubsection{Aromatic amines and heterocyclic amines}

The metabolism of aromatic amines is rather complex. They may be converted to an aromatic amide catalyzed by acetyl co-enzyme A dependent acetylation or may undergo Noxidation to give N-hydroxylated products which may subsequently be protonated by acidic conditions in the urinary bladder giving rise electrophiles capable of binding DNA. The initial activation step of both aromatic amines and amides is through CYP1A2 mediated Noxidation (Weston et al. 2005).

Heterocyclic amines are bioactivated by CYP1A2 mediated N-hydroxylation followed by enzymatic O-esterification. The nitrenium ion is likely to be the ultimate carcinogen capable of adduct formation (Goldman et al. 2003).

\subsubsection{Aflatoxins}

Aflatoxins may be activated by various CYPs (CYP2A3, CYP1A2, CYP2A6, CYP3A4, and CYP3A5). In case of aflatoxin B1, the process involves a CYP3A4 or CYP1A2 mediated oxidation at the exo 8, 9 position to form an electrophilic exo 8, 9 epoxide which can subsequently bind to DNA and proteins and form adducts. Formation of these adducts has been shown to cause G:C to T:A transversion at codon 249 of the p53 tumor suppressor gene (Kensler et al. 2004). Formation of CYP1A2 mediated aflatoxin M1 is considered a detoxification pathway (Sudakin 2003).

\subsubsection{N-Nitrosamines}

Endogenous nitrosamines form during the reaction of an amine with a nitrate alone or a nitrite in the presence of an acid. Dietary and tobacco specific nitrosamines constitute some 
of the exogenous sources of nitrosamines. N-nitrosodimethylamine formed from exposure to nitrosamines undergo enzymatic $\alpha$-hydroxylation (CYP2E1/ CYP2A6 mediated) to form an unstable $\alpha$-hydroxynitrosamine which subsequently hydrolyzes to an aldehyde and a monoalkylnitrosamine. The monoalkylnitrosamine can subsequently release a carbocation which can alkylate DNA bases.

\subsection{DNA damage}

Exposures to carcinogens through various routes lead to DNA damage. The damage may involve formation of addition compounds by covalent attachment to DNA bases (adduct formation), alterations in DNA structure (single and double strand breaks, DNA strand crosslinks, chromatid exchanges), oxyradical damage of bases, transitions, transverisons, dimerization, deamination as well as epigenetic changes (Yuspa et al. 1997; Weston et al. 2005).

Most carcinogens are mutagens causing heritable changes in the structure of DNA. Different nucleophilic sites on DNA form targets for attack by different adduct forming species. These include N1, N3 and N7 of adenine, N3 of cytosine, O2, N3 and O4 of thiamine and N2, O6 and N7 of guanine. DNA adduct formation may involve the addition of just a portion of the chemical carcinogen such as a small alkyl group (as in the case of carcinogens like N-nitrosamines, N-alkylureas, mustards and haloalkanes) or the entire carcinogen leading to the formation of large, bulky DNA adducts (as in case of many PAHs) (Weston et al. 2005). In many cases these changes lead to the formation of 'signature' mutations in certain key oncogenes and /or tumor suppressor genes. For example, in many cases of tobacco smoke induced lung cancer the common targets for adduct formation appear 
to be the ras oncogene as well as the p53 tumor suppressor gene. Most cases of aflatoxin induced hepatocarcinomas (from either China or South Africa) were found to harbor $\mathrm{G} \rightarrow \mathrm{T}$ transverisons in the $3^{\text {rd }}$ position of codon 249 of p53 (Yuspa et al. 1997; Weston et al. 2005). Activated benzopyrenes bind preferentially to the exocyclic amino group of deoxyguanosine (N2) to form (7R)-N2-(10[7b, 8a, 9a-trihydroxy-7,8,9,10-tetrahydrobenzo[a]pyrene]yl)-deoxyguanosine (BPdG) adducts which appears to be the most common adduct of BP though adducts with other bases in DNA have also been reported. With regard to aromatic amines the $\mathrm{C} 8$ of deoxyguanosine appears to be the predominant site for adduct formation though adducts with C8, N2 and $\mathrm{O} 6$ are also possible (Weston et al. 2005). Heterocyclic aromatic amines also have a preference for the $\mathrm{C} 8$ and amino groups of purines, especially deoxyguanosine for forming adducts (Dipple 1995). Aflatoxin B1 and G1undergo oxidation at the 8, 9-position to form N7 deoxyguanosine adducts. This in turn could undergo ring opening to form two pyrimidine adducts $\mathrm{N}$-nitrosamines upon metabolic activation bring about alkylation of DNA at some of the similar sites on DNA bases as mentioned above though the N7 of guanine appears to be the preferred site.

Apart from these, oxidative damage of DNA from various endogenous and/or exogenously sources of oxygen radicals can lead to the formation of thymine glycol or 8hydroxydeoxyguanine adducts. The catalytic cycling of BP, endogenous lipid peroxidation and catalytic cyclic of some enzymes include some of these (Guengerich 2001). Etheno adducts can result from reaction of the bases in DNA with xenobiotics such as urethane and chemicals such as carbon tetrachloride and ethanol. 


\subsubsection{DNA repair}

Maintenance of the genome integrity may be achieved at various levels such as by prevention of carcinogen activation, prevention of interaction of the activated carcinogen with the target site, abrogation of the damage caused, or various combinations of these. Impaired capacity for DNA repair is associated with various cancers and syndromes. More than 140 gene products are implicated to play roles either directly or indirectly in different mechanisms of DNA repair mechanisms (Knowles et al. 2005). There are six main mechanisms of DNA repair: direct DNA repair, nucleotide excision repair (NER), base excision repair (BER), mismatch repair, non-homologous end joining and homologous recombination (Yuspa et al. 1997; Weston et al. 2005).

Generically DNA repair involves damage recognition, excision of the damaged strand, resynthesis and ligation. All these repair mechanisms involve multiprotein complexes with many of their components being involved in common in more than one repair process (Weston et al. 2002).

Direct DNA repair involves alkytransferases which remove alkyl moieties from alkylated bases followed by transfer of the moiety to a cysteine in the active site of the repair enzyme. The best known example of this mechanism is the removal of $\mathrm{O}^{6}$-methyl guanine.

NER and BER are similar with both involving multi-protein complexes which recognize the damage, remove the damage, fill in the gaps created and finally seal the nick. NER however, is employed in the repair of large bulky adducts such as those of benzopyrenes while BER is typically involved in the removal of smaller alkyl groups.

DNA mismatches involving single base mismatches due to errors in DNA repair or replication can be corrected by mismatch DNA repair mechanism which involves removal of 
large pieces of mismatched DNA using protein complexes that bind to the mismatch and the nearest unmethylated adenine in the sequence GATC, followed by resynthesis of excised part by DNA polymerase and finally ligation by a ligase complexed to the polymerase.

Large bulky adducts such as those of BP can also distort DNA causing it to break. In addition, other mechanisms such as exposures to ionizing radiations and a damage tolerance mechanism known as post explicative repair also causes DNA double strand breaks. These double strand breaks may be repaired by homologous or non-homologous recombination mechanisms. Homologous recombination involves the simultaneous resection of both strands followed by invasion of the homologous duplex by 5 ' tails of the disrupted DNA, resynthesis followed by polymerization and ligation.

Non-homologous end joining is a repair mechanism that is homology independent. The breaks may be symmetric or asymmetric; in case it is asymmetric resection occurs to create blunt ends before they are joined. This method of repair is therefore prone to introduction of small deletions in the sequence of the repaired DNA.

\subsection{Potential impact on hormonal carcinogenesis}

$\mathrm{BP}$ is an inducer of steroid hydroxylases, CYP1A1 and CYP1B1, which in turn bring about the metabolism of BP to reactive intermediates. These enzymes along with CYP3A4 also play a vital role in the oxidative metabolism of two of major natural estrogens, estrone (E1) and estradiol (E2) (produced by the ovaries) as well as other synthetic estrogens such as $17 \alpha$ ethyinylestradiol (Liehr et al. 1990). The major pathway of estrogen oxidation involves their P450 mediated hydroxylation to form catechol estrogens (CEs), their 2-OH and 4-OH derivatives, and 16 $\alpha-\mathrm{OH}$ metabolite (Cavalieri et al. 1997). Although these CEs can be 
inactivated through conjugation, their incomplete or ineffective conjugation may lead to the production of reactive semiquinones and/or quinones radicals. These quinones can subsequently be inactivated by conjugative mechanisms. However, incomplete removal may allow them to bind to DNA and form stable DNA or depurinating DNA adducts. The formation of $\mathrm{N} 7$ guanine adducts by CE-3,4-quinone is thought to be a tumor-initiating event (Cavalieri et al. 1997). Apart from this, free radicals generated from the quinonesemiquinone redox cycling of estrogens can lead to free radical damage (Kirkman 1959; Liehr et al. 1990). Further, the 4-OH and 16 $\alpha-\mathrm{OH}$ metabolites of estrogen are found to have a potent cell proliferative effect in estrogen responsive tissues

\subsection{Chemoprevention}

Given the ubiquitous nature of benzopyrenes a complete avoidance of their contact is impossible. An alternative more practical approach may therefore involve reducing their uptake, preventing their metabolism or preventing the activated metabolites from reaching/interacting with the target site. The use of agents (natural, synthetic, biological or chemical) which may reverse, suppress or prevent the carcinogenic process at various phases of the multistep carcinogenic process may form a viable strategy. This constitutes the concept of chemoprevention. Though known for about four decades or so a greater insight into the idea was brought by two pioneers in the field, Lee W.Wattenberg and Michael B. Sporn. Initially called 'chemoprophylaxis of carcinogenesis' by Wattenberg (Wattenberg 1966), the term 'chemoprevention' was coined only later by Sporn et al., (1976). The original concept of chemoprevention was to inhibit or delay the occurrence of cancers by agents that would block one or more pathways in the induction of cancer. However, the concept has expanded 
over the years to include agents which would even reverse the carcinogenic process by transforming malignancy to pre-malignancy.

\subsubsection{Chemopreventive agents}

Currently there are approximately 400 compounds being studied as potential chemopreventive agents of which around $10 \%$ are in clinical trials (http://www.cancer.gov/cancertopics/factsheet/Prevention/chemoprevention).

Chemopreventive agents can be classified into 4 main categories: blocking agents, agents reducing tissue vulnerability to carcinogenesis and suppressing agents (Wattenberg 1997).

Blocking agents act through prevention of carcinogen activation, enhancement of carcinogenic detoxification or prevention of activated metabolites from reaching critical target sites. Agents reducing tissue vulnerability to carcinogenesis act through effecting cellular maturation, reducing the activity of the target cells, decreasing cell proliferation or modulating signal transduction and hormone/growth factor activity.

Suppressing agents prevent the evolution of the neoplastic process in cells that may be on the verge of becoming malignant. Some suppressing agents may act by producing differentiation and inhibiting cellular proliferation. Another large group of compounds not fitting into any of these categories have the capacity to inhibit components of the arachidonic acid pathway and induce antioxidant enzymes.

Ideal chemopreventive agents are ones which combine multiple mechanisms of action, have low toxicity, high efficacy, are easily acceptable in intervention strategies and are economically viable (Egner et al. 2001). 


\section{Chlorophyllin (CHL)}

Porphyrins are compounds with four pyrrole nuclei (a five-membered heterocyclic aromatic ring compound with the formula $\mathrm{C}_{4} \mathrm{H}_{5} \mathrm{~N}$ ) joined by -CH- bridges.

Porphyrins, both natural as well as modified versions are generally, effective antimutagens and anticarcinogens against heterocyclic amines and various other mutagens possessing polycyclic structures. Hemin (the natural porphyrin found in hemoglobin and myoglobin), copper pthalocyacnin (a blue pigment similar in structure to hemin) and chlorophyll (the green pigment in photosynthesizing plants) have all been found to be effective antimutagens (Hayatsu 1995). The structures of these compounds are presented in Figure 4.

Chlorophylls and even more so CHL have been gaining prominence over the last decade as effective chemopreventive agents (Dashwood 1997b). Commercially available CHL consists mainly of a trisodium copper salt apart from small amounts of mono- and di-carboxylic acid chlorins (Dashwood 1997b).

Though several medical and clinical uses of CHL exist (Kephart 1955) its increasing use in cancer biology stems from its ability to exhibit various activities in in vitro and in vivo studies making it an attractive chemopreventive agent. Its has been found to be antimutagenic being effective against various chemicals such as aflatoxin B1, BP, heterocyclic amines, coal dust and airborne particles (Terwel et al. 1985; Ong et al. 1986; Dashwood et al. 1991; Warner et al. 1991). Anticlastogenic activities of CHL have been demonstrated in a few studies (Sarkar et al. 1996; Negraes et al. 2004). CHL has also been shown to exhibit antigenotoxic (Bez et al. 2001; TorresBezauri et al. 2002), anticarcinogenic (Park et al. 1996; Reddy et al. 1999), antioxidant (Kamat et al. 2000; Kumar et al. 2004)) apoptosis inducing (Diaz et al. 2003; Chiu et al. 2005) and immuno modulatory properties (Yun et al. 2005; Sharma et al. 2006). 
<smiles></smiles>

Hemin

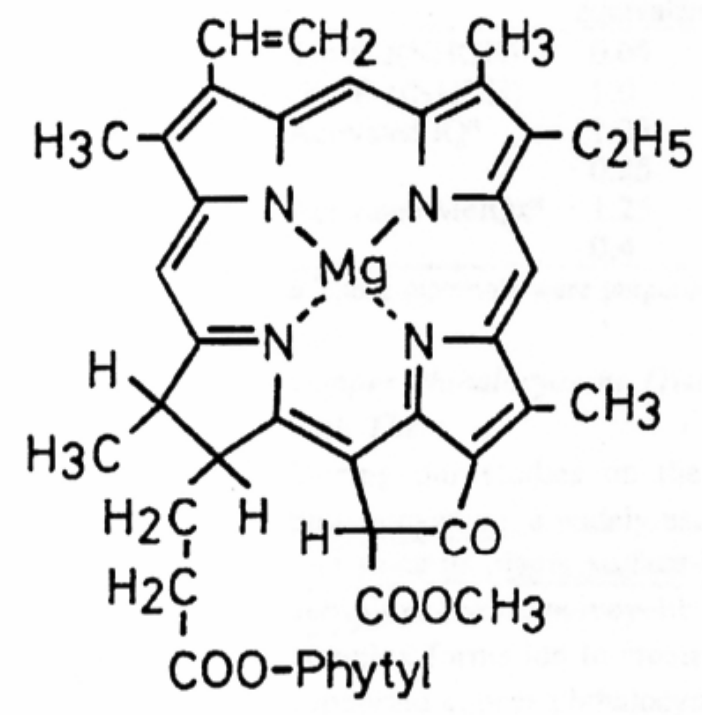

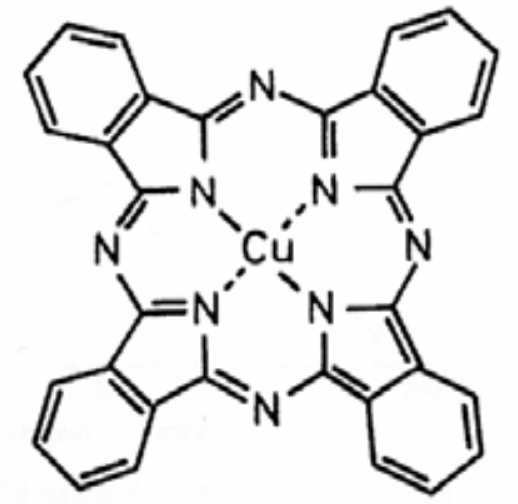

$\mathrm{Cu}$-pthalocyanine

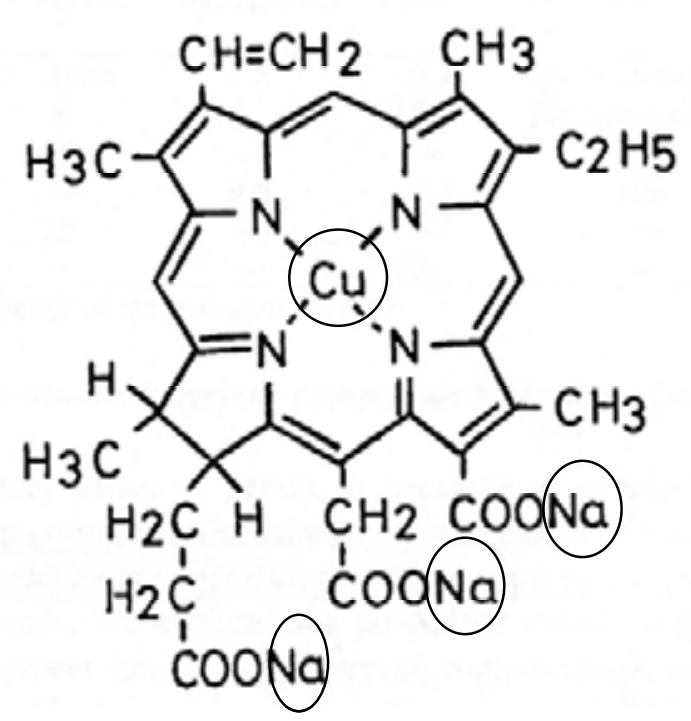

\section{Chlorophyll}

\section{Chlorophyllin (CHL)}

Figure 4: Structures of some of the biologically important metalloporphyrins. The groups that differ in CHL compared to chlorophyll are circled. The positions occupied by sodium (Na) can also be occupied by potassium $(\mathrm{K})$ depending on reaction conditions during the conversion of chlorophyll to CHL. Commercial CHL is mainly the trisodium salt shown here along with small amounts of mono-sodium and di-sodium salts. 
The most conclusive evidence of CHL as an anticarcinogenic agent probably comes from its role in the chemoprevention of hepatocarcinoma (liver cancer) in aflatoxin endemic areas of the world such as China (Kensler et al. 2004). Chronic hepatitis B virus (HBV) infection along with exposure to aflatoxins through diet contributes to extremely high risk of liver cancer in Quidong. Exposure to aflatoxins is also widespread in Beijing, Taiwan, certain parts of Africa and other developing areas of the world leading to high risk of liver cancer of exposed populations. While primary intervention through HBV vaccination has helped to some extent, secondary intervention strategies through the use of CHL or other drugs such as oltipraz have greatly helped reduce the risk of disease through various mechanisms discussed below. Administration of CHL helped reduce AFB1- N7-guanine adducts by as much as 55\%. In this aspect CHL has an advantage over other agents such as oltipraz, given its low cost and high efficacy leading to its easy adoptability by a larger section of at risk human populations in developing areas (Sudakin 2003; Kensler et al. 2004).

However, there are conflicting reports with regard to the use of chlorophyll or CHL as chemopreventive agents of choice with some studies pointing to chlorophyll being a better choice (de Vogel et al. 2005) others CHL (Terwel et al. 1985) and yet others showing both to be equally effective (Bez et al. 2001). The nature of the carcinogen, system under study and end points observed may contribute to some of the differences. Again, with respect to CHL, there are some studies which point not only to the lack of a positive modulatory effect (Torres-Bezauri et al. 2002) but also to an enhancement of the tumor promotion (Nelson 1992) or increase in mutation frequency (Negraes et al. 2004). In another study, CHL when administered after or during and after carcinogen exposure (1,2 dimethyl hydrazine) 
enhanced the number of colon tumors produced in rats. Additionally, in the same study, when a range of $\mathrm{CHL}$ concentrations were administered through drinking water, following exposure to 1,2 dimethyl hydrazine (DMH) or 2-amino-3-methylimidazo[4,5-f]quinoline (IQ), the lowest concentration of CHL (0.001\%) enhanced the multiplicity of DMH induced tumors while having no promotion effect on tumors induced by IQ (Dashwood et al. 2001). Thus, here too the test species involved, carcinogen used, the concentration of CHL employed, the route of administration and temporal aspects of the exposure protocol may all be key factors playing an important role in deciding the final role of $\mathrm{CHL}$ as an inhibitor or a potentiator.

\section{Mechanisms of action}

Several mechanisms of CHL action have been proposed. By acting as a suicidal inhibitor, CHL has been shown to bring about non-specific inhibition of CYPs probably involving destruction of the heme prosthetic group of CYPs (Yun et al. 2005). However, other investigations involving spectral studies have proposed that the inhibition may be rather indirect by interacting with NADPH-cytochrome P450 reductase, a rate limiting enzyme in mixed-function oxygenase system rather than involving any direct interaction with P450. This interaction may involve a physical interaction of CHL with NADPH-cytochrome P450 reductase preventing docking $\mathrm{P} 450$ with or its role as an alternative electron acceptor interfering with the electron flow from NADPH to P450s (Tachino et al. 1994). In addition to inhibition of Phase I enzymes, CHL has also been reported to enhance the expression of Phase II detoxification enzymes. One study revealed that following oral administration, CHL enhanced the amounts of hepatic glutathione S-transferase in lactating mice (Singh et al. 1996). Studies on induction of NADPH quinone reductase (NQO1), a 
quintessential Phase II enzymes showed CHL and chlorophylls to be significant inducers of NQO1 (Fahey et al. 2005).

The most widely accepted mechanism is the role of CHL as an 'interceptor molecule' or 'desmutagen' wherein CHL in addition to acting as a free radical scavenger also forms tight molecular complexes with the activated carcinogen preventing it from interacting with DNA or other cellular macromolecules (molecular trapping). Studies indicate that the mutagen should possess at least 3 fused rings with some degree of planarity in order to complex with CHL (Arimoto et al. 1993). Two forces play a key role in complex formation; van der Walls forces between overlapping ring systems of the mutagen and CHL and ionic or $\mathrm{H}$ bonds between substituents on the mutagen and carboxyl groups of the inhibitor (Dashwood et al. 1996). In case of BP, hypothetical complexes between CHL and BPDE (benzo[a]pyrene -trans-7, 8-dihydrodiol-9, 10-epoxide) are thought to be stabilized by multiple $\pi-\pi$ interactions between CHL and BPDE. Following sequestration of the activated carcinogen (BPDE) by CHL they may be degraded to more hydrophilic tetrols and excreted (Tachino et al. 1994).

A relatively recent study has also shown the role of CHL as a modulator of carcinogen uptake and transport. The study examining the effects of a range of CHL concentrations on the transport of 3 different carcinogens, dibenzo(a,1)pyrene (DBP), 2amino-1-methyl-6-phenylimidaza-[4,5-b]pyridine (PhIP) and AFB1 across Caco-2 cell monolayers from the apical to the basolateral side found that all concentrations of CHL reduced the transport of DBP from the apical to the basolateral side though not much effect was observed on the transport of the other two carcinogens (Mata et al. 2004). Though there have hardly been any further studies on this aspect of CHL, it may be possible that CHL may 
have a modulatory effect on the intake and transport of BP. CHL has been reported to be a membrane stabilizer and hence it may be reasonable to make the above assumption (Dhir $1989)$.

As further mechanistic studies and intervention trials are conducted with CHL a greater number of mechanisms of action and properties of CHL may be unveiled.

\subsection{Cytochromes P450 (CYPs)}

Most carcinogens (including benzopyrenes) requiring metabolic activation to render them carcinogenic and are often bioactivated by various members of the cytochrome P450s. They enzymes possess some of the broadest range of substrate specificities with some being highly specific (such as those involved in steroid anabolism, mitochondrial and bacterial P450s) and yet others (such as those involved in oxidation of various substrates) exhibiting a broad range of catalytic activities (Guengerich 1991; Ioannides 1996; Lewis 2001). These proteins have been reported to be present in most tissues though mainly concentrated in the liver (1-2\% of an individual hepatocyte by weight) and localized to the endoplasmic reticulum (12-15\%) and to some extent the mitochondria. Some P450s have also been reported to be translocated outside the plasma membrane where they may have role in development of immune responses. As of 2005 there were reportedly $>4500$ named P450 genes (http://drnelson.utmem.edu/CytochromeP450.html).

Structurally, all P450s seem to contain an 8-10-residue signature motif (F G/S x H/R $\mathrm{x} \mathrm{C} \times \mathrm{G} / \mathrm{A}$ ) with a highly conserved cysteine residue serving as a ligand for a central heme (Lewis 2001). The central heme is bound to the four pyrrole nitrogens leaving room for two additional ligands in the axial positions. One of these ligands above the porphyrin plane is 
satisfied by the invariant cysteine and the other ligand below the plane generally being a hydroxyl group related to a tyrosinyl radical or an exchangeable water molecule (Janig et al. 1984; Porter et al. 1991).

P450s are mainly mixed function oxygenases which require a source of electrons to function. The electrons which are generally derived from NADPH are delivered to the iron in the CYP by a flavoprotein, NADPH cytochrome P450 reductase in case of P450s bound to the endoplasmic reticulum or by another protein, ferrodoxin reductase (adrenodoxin reductase in case of the adrenals) via an iron-sulfur cluster called ferrodoxin to mitochondrial P450s (http://drnelson.utmem.edu/CytochromeP450.html). In some less common cases, the routing of electrons from NADPH is may be carried out through cytochrome b5 and NADPH cytochrome b5 reductase or microsomal heme oxygenase (Shen et al. 1989).

The CYP superfamily is divided into families, subfamilies and individual genes based on primary amino acid sequence similarities. P450 protein sequences with $\leq 40 \%$ amino acid sequence identity are grouped into different families. Members within a family are divided into sub-families and individual genes (http://drnelson.utmem.edu/CytochromeP450.html). P450s catalyze the metabolism of various endogenous and exogenous substrates. In general, P450s in most non-animal species seem to be involved chiefly in the metabolism of endogenous substrates. Different P450 isoforms have been found to be involved in the biosynthesis and metabolism of steroids, fatty acids, prostaglandins, prostacyclins, thromboxanes, Vitamin D, bile acids and also in the metabolism of a large number of drugs (http://medicine.iupui.edu/flockhart/). 


\subsubsection{Regulation of CYPs}

The amount of functional P450s is subject to considerable inter-individual variation. Several factors such as the presence of polymorphisms in P450 genes, use of different nuclear receptors, post-transcriptional (mRNA stabilization) and post-translational (enzyme stabilization) mechanisms appear to play a role to regulate the abundance and activity of P450s (Figure 5). Most CYPs however appear to be regulated at the transcriptional level and many seem to be subject to tissue specific regulation.

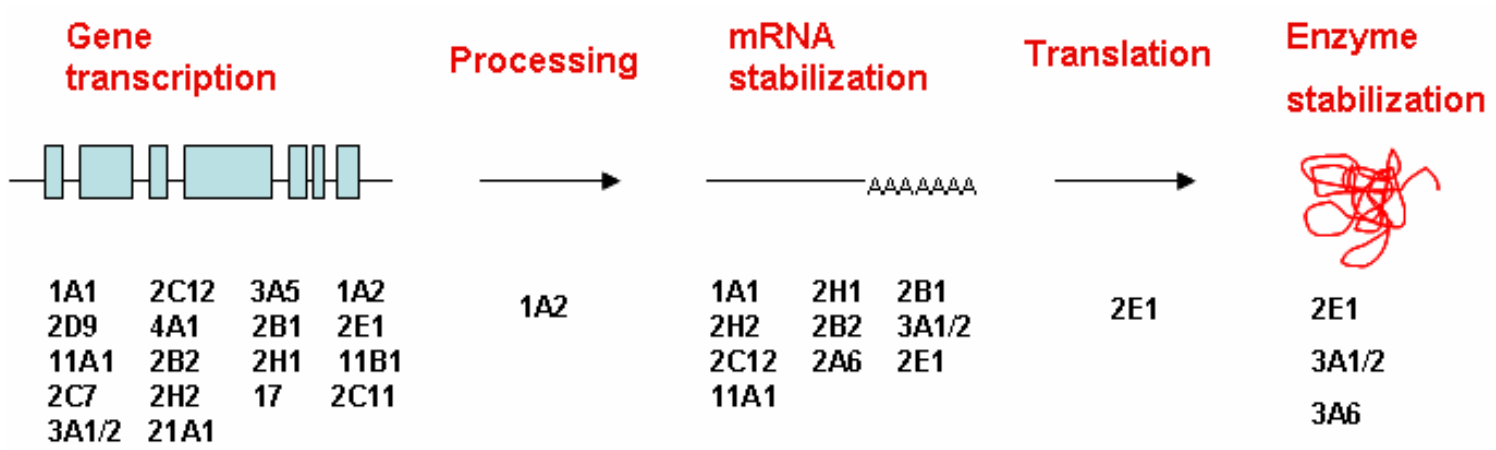

Figure 5: Multiple levels in the regulation of P450s. The different levels at which CYPs are regulated are indicated in red. The specific CYP isoform(s) regulated by the respective mechanism(s) is indicated below each mechanism.

The most well characterized $\mathrm{P} 450$ regulatory mechanisms are with respect to members of the CYP1 family, regulated by the Ahr-Arnt mechanism (discussed in detail in the section on $\underline{\mathrm{BP} \text { in 2.4.1). }}$.

Transcriptional regulation of the CYP2 family involves various receptors related to the nuclear receptor family such as the constitutive androstane receptor (CAR) in the case of CYP2B and the retinoic acid receptor (RAR) in the case of CYP2C (Lewis 2001). In 
addition, members of the CYP2B family are also regulated by protein kinase A (PKA). mediated phosphorylation (Oesch-Bartlomowicz et al. 2003). The regulation of CYP2A and CYP2D however, are, mediated by HNF-4 (hepatic nuclear factor-4). The regulation of alcohol inducible CYP2E1 appears to take place at multiple levels including posttranscriptional and post-translational. Diabetes and fasting appear to have a stabilizing effect on CYP2E1 mRNA. Presence of chemical inducers also stabilize the protein by preventing its phosphorylation by PKA and subsequent degradation (Oesch-Bartlomowicz et al. 2003).

CYP3 family members are mainly regulated by members of the orphan nuclear receptor family, glucocoticoid receptor (GR) and pregnane-X-receptor (PXR).

The CYP 4 family is regulated via another nuclear receptor, peroxisome proliferator activated receptor (PPAR).

Various other receptors involved in the modulation of different P450s associated with the metabolism of different endogenous substrates are present in Table 1.

Other mechanisms of regulation include regulation by cyclic AMP (cAMP) in the case of trophic hormones (Porter et al. 1991), use of cis and trans acting elements along with co-activator and repressor proteins, cross-talk between specific nuclear receptors (Lewis 2001) and in some rare cases by neonatal imprinting (Porter et al. 1991). In addition, certain chemicals can inhibit P450s through several mechanisms such as heme ligation, heme adduct formation, competitive inhibition, formation of reactive intermediates or various combinations of these and can therefore modulate CYP expression (Lewis 2001).

Polymorphisms in P450s have mostly been addressed in the context of 'drug metabolizer' phenotypes and susceptibility to diseases. However, the underlying cause of these is the differential expression of the respective P450s caused by the respective 
polymorphisms. For example, $* 2 A$ and $* 2 B$ variant alleles of $C Y P 1 A 1$ gene have a T3801C base change introducing a new $M s p I$ restriction site in intron 6 . These variant alleles are associated with enhanced enzyme inducibility and subsequently an elevated risk of smoking associated lung cancer in Japanese and Caucasian cancer patients (Chowbay et al. 2005). The P450s form one of the most polymorphic groups of enzymes. A comprehensive list of different polymorphisms in P450 enzymes can be found at (http://www.imm.ki.se/CYPalleles/).

In summary, the overall amounts of P450s are governed by the concentration of inducers and repressors, polymorphisms, and cross-talk with various other receptors, ligands, proteins, and signaling molecules with other factors within the cell. 
Table 1: List of receptors and factors associated with the induction of some of the P450s involved with endogenous substrate metabolism

\begin{tabular}{ccc}
\hline CYP & Receptor/Factor & $\begin{array}{c}\text { Trophic hormone as } \\
\text { mediating factor }\end{array}$ \\
\hline 7 & FXR, LXR & - \\
11 & SF-1 & ACTH (adrenals), LH \\
& & (testis) \\
17 & SF-1 & ACTH (adrenals), LH \\
19 & SF-1, ER & FSH (ovary) \\
21 & SF-1 & ACTH (adrenals), \\
26 & RAR, RXR & PTH (kidney) \\
27 & VDR &
\end{tabular}

$\mathrm{FXR}=$ farnesoid X receptor (down-regulator of bile acid synthesis), LXR = liver X receptor (up-regulator of bile acid synthesis), $\mathrm{SF}=$ steroidogenic factor $1, \mathrm{ER}=$ estrogen receptor, $\mathrm{VDR}=$ vitamin $\mathrm{D}$ receptor, $\mathrm{ACTH}=$ adrenocorticotrophic hormone, $\mathrm{LH}=$ lutenizing hormone, $\mathrm{FSH}=$ follicle stimulating hormone, $\mathrm{PTH}=$ parathyroid hormone

\subsection{Biomarker Concept}

Traditional epidemiology has investigated the relationship between exposure and disease. The extent that identification of preclinical changes was possible assisted the better understanding of that relationship. Before the introduction of biomarkers the intermediary steps in the exposure-disease progression were considered to be unknown (a "Black Box"). The introduction of biomarkers has assisted greatly in the effort to see into the "Black Box." 
(Hirschhorn et al. 2002; Wogan et al. 2004). The biomarker concept in molecular epidemiology has been refined significantly since it was articulated by Perera and Weinstein in 1982 (Perera et al. 1982; Perera et al. 2000) and promises to elucidate a large number of intermediary steps that have the potential to impact upon risk of disease as depicted in the schematic representation below.

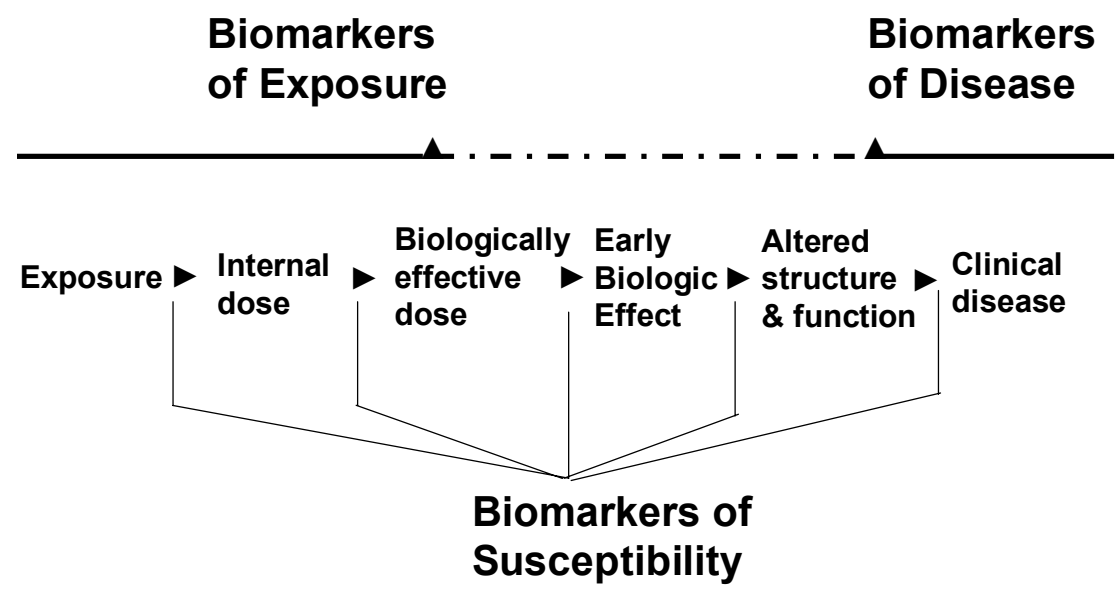

Schematic representation of the components of the exposure-disease continuum emphasizing the need for development of biomarkers of human cancer risk at every pathologic stage 
A biomarker is a characteristic that is objectively measured and evaluated as an indicator of normal biologic processes, pathogenic processes or pharmacological responses to therapeutic intervention (Srivastava et al. 2002). In order for biomarkers to be valuable they need to be sensitive, specific and predictable in the manner they change between the normal and the diseased states.

Human chemical carcinogenesis generally has a long latency. For decades, a pathology approach has used microscopy of biopsied slides to understand structural changes underlying the transformation process. Unfortunately, when these discernable or measurable changes have occurred many molecular changes have also occurred and it is usually too late for successful intervention (Negm et al. 2002).

Emphasis needs to be on the development of early biological markers of the disease process prior to the transformation of normal cells. Detecting and understanding these changes quickly and accurately will he greatly aid in the early detection of cancers. In addition it will also help shape preventive and therapeutic measures and also predict prognostic outcomes.

Conventional epidemiology, which draws on casual associations between certain exposures/lifestyles and risk of certain cancers, requires studies of population risks and may at best help identify and monitor other individuals or populations at risk; however, it cannot help early detection of cancer. Recent technological advances have helped in a greater understanding of the cancer process at the molecular level and in turn has helped develop biomarkers for early detection and risk assessment of different exposure scenarios (Srivastava et al. 2002). 
Molecular epidemiology (the use of molecular and biochemical tools to detect early molecular changes associated with cancer risk) can now be used in conjunction with conventional epidemiological studies to detect some cancers in their early stages (Weston et al. 2005). Thus, they are intended to supercede clinical endpoints.

\subsubsection{Types of cancer biomarkers}

According to schematic representation above there is a hierarchy of biomarkers: general exposure biomarkers include metabolites in urine, saliva and other body fluids (e.g., cotinine and cortisol signaling exposure to nicotine and stress, respectively); biomarkers of biologically effective dose include carcinogen-protein or carcinogen-DNA adducts derived from blood (these are surrogates for potential genetic damage in the DNA of target tissues). BP-DNA adducts have been valuable in the integration of dose and biological propensity of DNA damage from PAHs (Perera et al. 1982). Exfoliated aflatoxin-guanine adducts in urine (AFB1-N7-dG) have been identified as a risk factor for hepatocellular cancer in populations potentially exposed to AFB1 in china and S. Africa (Kensler et al. 2004).

In conjunction with these studies, there is the possibility to assess cancer susceptibility based on genetic variation. When the continuum of events from exposure to disease is considered, it should be noted that the contribution from the Human Genome Project (HGP) has been highly significant in the arena of genetic polymorphisms. Recent studies have shown that molecular epidemiologic association studies offer a powerful approach to the identification of genetic variants that influence disease susceptibility (Hirschhorn et al. 2002; Consortium 2003). Some of these pertain to potential markers of 
susceptibility in the context of a specific exposure scenario, for example: bladder cancer and the NAT2 genotype in dye workers and in tobacco smokers (Green et al. 2000).

Gene expression patterns have been used to compare different types of tumor, different tumor grades and tumor response to treatment (Lonning et al. 2005). However, from the perspective of carcinogenesis, recent studies have identified gene expression patterns within the continuum of the carcinogenic process. One such study described gene expression patterns in peripheral white blood cells in response to exposure in tobacco smokers (Lampe et al. 2004).

In addition to gene expression studies, gene mutation studies have been important biomarkers that have been used for both understanding etiology (Olivier et al. 2004) and predicting outcome (Jackson et al. 2003). A prime example is that of p53 mutations. There is good evidence that exposure to specific agents results in specific or limited types of p53 mutations in certain types of cancer; for example AFB1 and liver cancer, polycyclic aromatic hydrocarbons and lung cancer, and UV irradiation and skin cancer (Olivier et al. 2004). From prospective studies of AFB1 related liver cancer, p53 mutations have been identified in fragments of tumor DNA exfoliated into blood serum before clinical manifestation of disease. There by providing a biomarker for early liver cancer detection in high AFB1 exposure regions of the world.

Many genes including oncogenes and tumor suppressor genes are regulated by epigenetic mechanisms. Dysregulation of epigenetic control of various genes such as p15, p16, APC and VHL have been observed to occur early on in several cancers (Negm et al. 2002). 
Other penitential biomarkers include measurement of chromosomal aberrations, point mutations and loss of heterozygosity in DNA from various body fluids of cancerous patients. Presence of anti-apoptotic proteins in the urine of patients with recurrent bladder cancer has been reported and has been found to be a useful biomarker (Smith et al. 2001).

\subsubsection{Challenges in using biomarkers}

In order for biomarkers to be used efficiently and reliably they will need to be highly sensitive and specific. Though there has been significant progress in the field of cancer biomarker research over the last two decades it has still not reached a level of confidence for application in the clinical setting, and validation studies are required. Some of the biomarkers currently in use such as prostrate specific antigen (PSA) for prostrate cancer, CA125 for ovarian cancer are not very specific for their respective cancers. In addition, in this time of rapidly advancing technological developments, there is a need for more effective as well as novel cancer biomarkers. 


\subsection{Statement of the problem}

Benzopyrenes, a class ubiquitous environmental contaminants are procarcinogens. Due to their widespread distribution, alternative strategies such as the use of chemopreventive measures to mitigate some of the genetic damage caused by these chemicals may form a practical approach. This study was designed to seek biomarkers of BP exposure (using microarrays) which could subsequently form targets for chemopreventive intervention. CHL, a water soluble metalloporphyrin was used as a chemopreventive agent in all these studies. Also, since benzopyrenes are procarcinogens requiring metabolic activation by CYP1 enzymes for their conversion to DNA damaging metabolites, the effect of CHL on mitigating the expression of $C Y P 1$ enzymes, in addition to their efficacy in preventing the interaction of the activated BP metabolites with cellular macromolecules such as DNA as measured by RT-PCR and BP-DNA adduct analysis, respectively was determined. B[e]P, a structural isomer of BP which has been reported to be weakly carcinogenic was also examined in parallel with BP (both in the absence and presence of CHL) to induce CYPI gene expression as well as cause DNA adduct formation. In addition, MCF-7, a malignantly transformed breast cell line was observed in parallel with NHMECs across different CHL treatments and concentrations to examine if their responses parallel those of normal cells and in turn whether they can be used as a surrogate to normal cells in studying carcinogenic stress. 


\subsection{MATERIALS AND METHODS}

Most experiments in this study were carried out on a panel of primary NHMECs developed from breast tissue discarded at reduction mammoplasty. In order to compare the responses of cancerous cells, a breast cancer cell line, MCF-7 was used in parallel with NHMEC(s) for some of the studies. Genome-wide changes in gene expression patterns were initially observed in a panel of 6 NHMECs. Subsequently, gene expression patterns of CYP1A1 and CYP1B1, two key BP metabolizing enzymes as well as the level of BP-DNA adducts was observed in a panel of 20 NHMECs. For studies on the comparison CYP1A1, CYP1B1 gene expression and the extent of DNA adducts on treatment of cells with BP or B[e]P, 2NHMECs (M98025 and M98015) were used. Studies for comparison of normal cells with malignantly transformed cells were carried out on a NHMEC strain (M00012) and the cancerous cell line MCF-7 over a range of CHL concentrations and treatments. An overview of the different experiments carried out in this study is shown in Table 2.

\subsection{Breast tissue}

Breast tissues from otherwise healthy women undergoing reduction mammoplasty were obtained from the Cooperative Human Tissue Network (CHTN) sponsored by the National Cancer Institute (NCI) and National Disease Registry Interchange (NDRI). Information on age, ethnicity of donors and a corresponding pathology report were obtained without personal identifiers. Since tissue samples were provided without personal identifiers the study was granted a waiver by the Human Studies Review Board at NIOSH. 


\subsection{Development of NHMEC strains}

NHMECs were isolated from the breast tissues by a process involving a combination of mechanical disruption, enzymatic digestion and filtration as described (Stampfer et al. 1980) with slight modifications. Briefly, stromal and epithelial tissue masses (following removal of fat deposits (by dissection) were minced to $1-2 \mathrm{~cm}$ pieces, filled into $50 \mathrm{ml}$

conical tubes up to $1 / 3^{\text {rd }}$ the capacity and incubated overnight at $37^{\circ} \mathrm{C}$, on a wave mixer with constant shaking with Tissue Digestion mix (Appendix). Following partial digestion the following day, the tubes were spun $(250 \mathrm{~g}, 10 \mathrm{~min}$ in a swinging bucket centrifuge), the supernatant removed and the tissue pellet resuspended in fresh Tissue Digestion mix and incubated for another 24-48h to ensure complete tissue digestion. Complete tissue digestion was ascertained by observing for organoids (cell clumps) under 40X and 60X objectives of an Olympus IX70 Inverted Fluorescent light microscope. Completely digested pellets were pooled together (typically 4 tubes into1) and resuspended in $5 \mathrm{ml}$ freezing media (Appendix). The resuspended pellets were then filtered through a $150 \mu \mathrm{m}$ nylon filter and the flow through refiltered through a $90 \mu \mathrm{m}$ nylon filter. The organoids retained on the $150 \mu \mathrm{m}$ nylon filter were resuspended in a small volume of freezing media (Appendix) to constitute the 150 $\mu \mathrm{m}$ ductal pool consisting mainly of a population of epithelial cells. Similarly, the organoids on the $90 \mu \mathrm{m}$ filter were resuspended in a small volume of freezing media to constitute the 90 $\mu \mathrm{m}$ fraction consisting of some small epithelial clumps. The $150 \mu \mathrm{m}$ fraction, $90 \mu \mathrm{m}$ fraction and the filtrate obtained after retention of the $90 \mu \mathrm{m}$ fraction were all separately aliquoted to cryogenic vials frozen in isopropanol containers at $-80^{\circ} \mathrm{C}$ overnight and then archived in liquid nitrogen until needed. 


\subsection{Cell culture}

NHMECs were grown and sub-cultured up to passage 6 . The $150 \mu \mathrm{m}$ organoids were seeded with a sterile Pasteur pipette to collagen treated flasks, allowed to stand for about 5 minutes and then supplemented with reconstituted serum-free mammary epithelial medium (MEBM; Clonetics ${ }^{\mathrm{TM}}$, Wakersville, MD) supplemented with manufacturer supplied Singlequots ${ }^{\mathrm{TM}}$ (gentamycin sulfate-amphothericin B, recombinant human epidermal growth factor, bovine insulin, bovine pituitary extract and hydrocortisone). Cell migration from the organoids was generally observed $24-48 \mathrm{~h}$ after seeding. Cells were allowed to reach $60-70 \%$ confluency before they were sub-cultured. Cells were maintained in MEBM mammary epithelial medium at $37^{\circ} \mathrm{C}, 5 \% \mathrm{CO}_{2}$ and $95 \%$ relative humidity. NHMECs are adherent cells and were sub-cultured by trypsinization. Cells at 60-70\% confluency were washed once with 1X PBS following removal of media, incubated with $0.025 \%$ Trypsin-EDTA $\left(\right.$ Clonetics $^{\mathrm{TM}}$, Wakersville, MD) at $2 \mathrm{ml}$ Trypsin-EDTA $/ 25 \mathrm{~cm}^{2}$ growth area of the flask and then incubated at $37^{\circ} \mathrm{C}$ for $2-3 \mathrm{~min}$ till $\geq 90 \%$ of the cells detached from the flask and appeared rounded when viewed under the microscope. At this stage the flask was gently rapped and the Trypsin-EDTA neutralized with Trypsin Neutralizing Solution (TNS) at $4 \mathrm{ml}$ TNS/25 $\mathrm{cm}^{2}$ growth area of the flask and the detached cells were transferred to a $50 \mathrm{ml}$ conical tube. The residual cells in the flask were collected by rinsing the flask with $1 \mathrm{X}$ PBS. The cells were centrifuged at $150 \mathrm{~g}$ for $6 \mathrm{~min}$ in a fixed angle centrifuge. The supernatant was aspirated, the cells washed with 1X PBS and cell pellet finally resuspended in MEBM mammary epithelial media. The suspended cells were then seeded into new flasks at 10,000 cells $/ \mathrm{cm}^{2}$ and maintained in MEBM mammary epithelial medium. In this manner, cells were passaged up to passage 6. MCF-7 cells were from ATCC and were grown, passaged and maintained in a 
manner similar to the NHMECs. MCF-7 cells were grown and maintained in MCF-7 media (Appendix).

\subsection{Cell treatments}

All treatments were carried out on a uniform population of $\sim 70 \%$ confluent cells at passage 6 . The concentration of $\mathrm{BP}$ in all experiments was $4 \mu \mathrm{M}$. The concentration of $\mathrm{CHL}$ used was $5 \mu \mathrm{M}$ for all experiments except for experiments involving a comparison of MCF-7 cells with a NHMEC across a range of CHL concentrations. For these CHL dose response studies, CHL was used at a concentration of $2 \mu \mathrm{M}, 3 \mu \mathrm{M}, 4 \mu \mathrm{M}, 8 \mu \mathrm{M}$ and $16 \mu \mathrm{M}$. In all these experiments the concentration of $\mathrm{BP}$ and $\mathrm{CHL}$ used were decided based on preliminary cytotoxicity assays. For studies on comparison of $\mathrm{BP}$ and $\mathrm{B}[\mathrm{e}] \mathrm{P}$ the concentration of $\mathrm{B}[\mathrm{e}] \mathrm{P}$ used was also $4 \mu \mathrm{M}$ in order to have a direct comparison with the effects of BP and was also dissolved in the same solvent as BP. BP was initially dissolved in acetone to obtain a $10 \mathrm{mM}$ solution and subsequently diluted to $4 \mathrm{mM}$ with absolute ethanol. The $4 \mathrm{mM}$ solution was stored in amber vials shielded from light at $-20^{0} \mathrm{C}$ until needed. Nuclease free water served as the solvent for CHL. For treatment of MCF-7 and NHMECs with $5 \mu \mathrm{M}$ CHL an initial stock solution of $5 \mathrm{mM}$ was prepared. For the CHL dose response study, $2 \mathrm{mM}, 3 \mathrm{mM}, 4 \mathrm{mM}, 8$ $\mathrm{mM}$ and $16 \mathrm{mM}$ solutions were prepared. All CHL solutions were prepared fresh just before use and shielded from light. These stock solutions of BP and CHL respectively were later diluted 1000 fold in MEBM mammary epithelial medium just prior to treatment of cells to obtain the desired micromolar concentrations. Acetone: ethanol (2:3) at 1:1000 dilution used as a solvent for BP served as the vehicle control. All treatments were carried out in duplicate $(n=2)$. 
The treatment protocol of 20 NHMECs and MCF-7 is detailed in Table 3. The treatment protocol for comparison of NHMECs on treatment with BP or B[e]P in the absence or presence of CHL is detailed in Table 4. The treatment protocol for the CHL dose response study comparing MCF-7 and a NHMEC (M00012) across a range of CHL concentrations is presented in Table 5.

\subsection{Expression analysis}

Analysis of genome-wide changes in gene expression patterns following exposure of NHMECs to BP in the absence or presence of CHL was carried out using microarrays. Subsequently, confirmation of gene expression patterns for a selected subset of genes which appeared altered on microarrays was carried out through real time polymerase chain reaction (RT-PCR). The magnitude and patterns of expression of $C Y P 1 A 1$ and $C Y P 1 B 1$, the main CYP isoforms known to be involved in the metabolism of $\mathrm{BP} / \mathrm{B}[\mathrm{e}] \mathrm{P}$ was also carried out through RT-PCR.

\subsubsection{Microarray analysis}

Arrays

Human genome U133A (HG-U133A) arrays containing 22,283 probe sets representing 14,500 genes were purchased from Affymetrix (Affymetrix, Santa Clara, CA). The sequences used in the design of these arrays were derived from GenBank, dbEST, and RefSeq. These arrays form a part of the HG-U133 set which consists of 2 arrays (HG-U133A and $\mathrm{HG}-\mathrm{U} 133 \mathrm{~B})$ and altogether consists of almost 45,000 probe sets representing 
approximately 33,000 well-substantiated human genes. HG-U133B mostly consists of ESTs and was therefore not used in this study.

Target generation

For microarray analysis due to considerations of cost and time involved only 3 treatment groups (solvent control (SC), BP, and pre CHL + post co-treatment) were considered across 6 NHMEC strains (M98035, M99005, M98015, M98025, M99025 and M99016).

\section{$\underline{\text { Total RNA isolation }}$}

Total RNA following treatment of cells was isolated using RNeasy kit (Qiagen, Valencia, CA) according to manufacturer's instructions. Cells following treatment were washed with 1X PBS, lysed in a chaotropic buffer containing guanidine isothiocyanate and homogenized in a spin column (Qiashredder) to shear high molecular weight DNA. The lysate containing total RNA was bound to a silica gel based membrane (RNeasy column) and the contaminants washed away using the manufacturer supplied wash buffers (RWE and RPE). Finally the bound total RNA was eluted out using RNAse free water.

$\underline{\text { cDNA synthesis }}$

$12 \mu \mathrm{g}$ of total RNA (260:280 ratio $>1.9$ and clear, non-smearing rRNA bands on agarose gel) were used for synthesis of double stranded cDNA using T7-oligo(dT) promoter primer kit (Affymetrix, Santa Clara, CA) and SuperScript Double-Stranded cDNA Synthesis kit, (Invitrogen Life Technologies, Carlsbad, CA) as per manufacturer's instructions. The generated double stranded cDNA was cleaned by phenol-chloroform extraction using Phase Lock Gels (PLG) and finally ethanol precipitated. The precipitated cDNA was dissolved in $12.0 \mu 1$ nuclease free water. 
$\underline{\text { cRNA synthesis }}$

A ‘one cycle' target labeling assay was used for generation of labeled target. $6.0 \mu 1$ of cleaned cDNA was used for the generation of biotin labeled complementary RNA (cRNA) target for hybridization on to HG-U133A arrays using the Gene Chip IVT Labeling Kit (Affymetrix, Santa Clara, CA). The generated cRNA was purified using RNeasy Kit (Qiagen, Valencia, CA) as per manufacturer's protocol. The cleaned cRNA was subsequently quantified using a Beckman DU 7500 UV/VIS spectrophotometer (Beckman Coulter, Inc., Fullerton, CA) to determine the yield and quality of the generated cRNA. An adjusted cRNA yield was calculated using the formula below for further down-stream processing.

Adjusted cRNA yield $=\mathrm{RNA}_{\mathrm{ivt}}-($ Total RNA $)(\mathrm{y})$

$\mathrm{RNA}_{\mathrm{ivt}}=$ amount of cRNA obtained following spectrophotometric analysis

Total RNA = starting amount of total RNA used for cDNA synthesis

$\mathrm{y}=$ fraction of the purified cDNA used for $\mathrm{cRNA}$ synthesis

The cRNA was subsequently fragmented to $35-200 \mathrm{nt}$ bases $\left(94^{0} \mathrm{C}, 35 \mathrm{~min}\right)$ prior to hybridization on to the arrays by metal induced hydrolysis using a 5X RNA fragmentation buffer (Appendix).

\section{Hybridization}

$15 \mu \mathrm{g}$ of fragmented cRNA were used for hybridization on to 'standard' Hu-Gene 133A arrays. The efficiency of target hybridization was assessed by incorporating pre-mixed, staggered concentrations of biotin labeled cRNA transcripts for three bacterial genes of the biotin synthesis pathway ( $b i o B, b i o C$, bioD) and one bacteriophage gene for recombinase (cre) at staggered concentrations of $1.5 \mathrm{pM}, 5 \mathrm{pM}, 25 \mathrm{pM}$ and $100 \mathrm{pM}$, respectively in a hybridization cocktail. A B2 oligo used as a positive hybridization control by the analysis 
software to place a grid over the scanned image and demarcate the probe area was also spiked into the hybridization cocktail. The remaining components spiked into the hybridization cocktail included herring sperm DNA, acetylated BSA, hybridization buffer and DMSO (Affymetrix 2004). The cocktail was heated to $99^{\circ} \mathrm{C}$ for 5 min prior to hybridization. The arrays with the hybridization cocktail were then incubated in a rotisserie oven at $45^{0} \mathrm{C}$ for $16 \mathrm{~h}$ with constant rotation $(60 \mathrm{rpm})$.

\section{Washing, staining and scanning}

The hybridized arrays were subsequently washed and stained on the GeneChip Fluidics Station 400 (Affymetrix, Santa Clara, CA) using Eu-GE-W2v4 protocol of Microarray Suite (MAS) 5.0 (Affymetrix 2004). A signal amplification protocol consisting of first using streptavidin phycoerythroprotein (SAPE) followed by a biotinylated antibody to SAPE and then SAPE again was used as opposed to a single stain protocol. Following washing and staining the arrays were scanned with an argon-ion laser on the HP GeneArray 2500 Scanner (Hewlett Packard, Palo Alto, CA). 


\section{Data analysis}

The scanning of arrays generated image files (.dat files) (Figure 6).

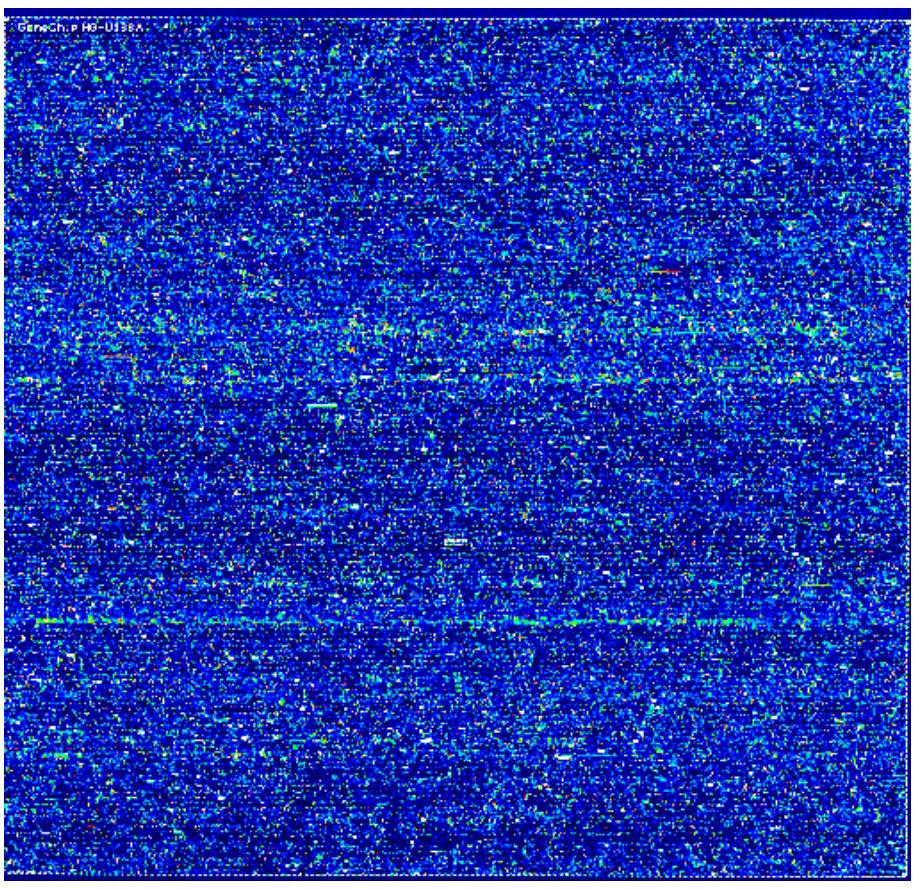

Figure 6: Representative scanned image obtained following scanning of arrays.

These .dat files were analyzed using MAS 5.0 (Affymetrix, Santa Clara, CA) to generate .cel files (with individual cell intensities), .chp files (with the analysis of cell intensities) and .rpt files (report files with an overview of various quality control parameters for each array/hybridization). Initially an 'absolute analysis' was conducted to compute absolute signal intensities of the SC as well as treated groups (BP and pre $\mathrm{CHL}+$ post cotreatment). 'Absolute analysis' involves analysis of each of the individual arrays to determine the presence or absence of a particular transcript (Detection call), measure their relative abundance (Signal) and also provide a 'Detection p-value' evaluated against a user defined cut-off to determine the Detection call. Two algorithms, a detection algorithm and a signal algorithm are employed to achieve this. 
Subsequently, a 'comparison analysis' comparing the fold change (FC) of the treated groups over the SC (BP versus SC and pre CHL + co-treatment versus SC) was also conducted. A 'change algorithm' which defines the nature of change (increase, decrease or no change) in expression trends of the experimental array as compared to its counterpart baseline array and another algorithm which provides a quantitative measure of the magnitude of change in terms of signal log ratio (SLR) are used for comparison analysis.

In this study, only probe sets having a Present call (P) and a FC $\sim 3.0$ or greater (SLR $\geq 1.5$ ) for up- regulated genes and SLR $\geq-1.5$ for down-regulated genes in both replicates in at least one of the 6 NHMECs were considered altered. Various quality control parameters such as background, noise, $3^{\prime} / 5^{\prime}$ GAPDH and $\beta$-Actin ratios, and $\%$ Present calls (between replicates) were examined in the .rpt files.

Graphical representation of all the altered genes were generated using Cluster and Tree View software (http://rana.lbl.gov/EisenSoftware.htm) using the average-linkage method (Sokal et al. 1958).

To identify common patterns of gene expression patterns across cell strains following different treatments 'coefficient of correlation clustering' was conducted using Micro DB 3.0 and Data Mining Tool (DMT) 3.0 (Affymetrix, Santa Clara, CA). In the 'correlation of coefficient' clustering, 'seeding', a pre-clustering process involving initial grouping of a small subset of genes with similar expression patterns to generate a 'seed' is first carried out with a user specified correlation coefficient threshold. Subsequently, a correlation coefficient threshold to assign probe sets to a particular 'seed' in order to create a 'cluster' is also defined (Affymetrix 2001). In this study, the correlation threshold of forming 'seeds' and assignment of genes to respective clusters were both set at 0.90 . Clustering was carried out to 
first find the genes common across all 6 cell strains on comparison of treated groups versus SC (BP versus SC and pre CHL + co versus SC). Only genes with a Present call (P) in both the replicates were used for the preliminary clustering. Subsequently, an unpaired, one-sided T test was carried out on above set of genes common to all 6 cell strains to find genes significantly altered due to respective treatment $(\mathrm{p}<0.05)$ and also determine the direction of change ('up' or 'down'). Gene expression 'cluster maps' were finally generated from the above subset of statistically significantly altered genes exhibiting particular expression pattern.

Due to the importance of host immune responses in better tackling genotoxic insults, cross-talks between immune response genes altered by SLR $\geq 1.5$ in at least one of the 6 NHMECs by the respective comparisons (BP versus SC and pre CHL + co versus SC) as well as any possible statistically significant associations of the altered genes with any of the presently well known and characterized biological pathways in GenMAPP, PharmGKB, KEGG and BioCarta was carried out using Pathway Studio v1.1 software (Ariadne Genomics, Rockville, MD) and ArrayXPath software (http://www.snubi.org/software/ArrayXPath), respectively.

Pathway Studio relies on a proprietary ResNet database to query nearly $500,000+$ functional relationships distilled from the entire PubMed and 43 full text journals to automatically extract information from scientific literature, and works with leading public and commercial databases of signaling and biochemical pathways, to construct cross-talks between the input genes/ proteins (http://www.ariadnegenomics.com). Initially, using Pathway Studio v1.1 all possible interactions of the altered immune response genes with all other processes, entities and proteins in the cell was deciphered. Later, 'shortest path' 
interactions were sought. Finally, only direct interactions, if any, between genes altered by the respective treatments were deciphered.

ArrayXPath is web-based software for mapping and visualization of microarray geneexpression data in the context of integrated biological pathway resources. A total of 45 pathways in GenMAPP pathways, 9 pathways PharmGKB pathways, 70 pathways in KEGG pathways and 346 pathways in Biocarta are queried by ArrayXPath to calculate associations of input genes with the respective pathways. A statistically significant association of the input gene(s) with alteration of the respective pathway is defined by a 'p' value (http://www.snubi.org/software/ArrayXPath). For genes involved in more than one pathway, a second $p$ value (in this case designated as q value) accounts for the multiple-comparison. In this analysis, both 'p' and 'q' values were set at $\leq 0.05$.

\subsubsection{RT-PCR}

Confirmation of gene expression on microarray was carried out through RT-PCR using 'SYBR green chemistry' for a selected sub-set of probes altered by SLR $\geq 1.5$ in at least one of the 6 NHMEC strains. $1 \mu \mathrm{g}$ of total RNA isolated from 3 treatment groups (SC, $\mathrm{BP}$ and Pre CHL + post co-treatment) using RNeasy kit (Qiagen, Valencia, CA) was reverse transcribed to single stranded cDNA using Advantage RT PCR kit (BD Biosciences, Palo Alto, CA) as per manufacturer's instructions

Primers for these genes were designed using Primer Express v1.5 (PE Applied Biosystems, Foster City, CA), got synthesized from Sigma-Genosys (Wodlands, TX) and used at 50 pmol concentration of each primer in a $25 \mu 1$ reaction mix with SYBR Green Universal PCR Master Mix (Applied Biosystems, Foster City, CA). Quantitation was carried 
out using a standard 96-well block on the 7900HT Fast Real-Time PCR System preloaded SDS software v2.2.2 (Applied Biosystems, Foster City, CA). Each sample was assayed in duplicate and their expression normalized to that of GAPDH using the $2^{-\Delta \Delta C T}$ method (Livak et al. 2001). The specificity of the generated product was confirmed by looking for a single specific product on ‘Amplify' software v1.2ß (University of Wisconsin, Genetics, Madison) prior to RT-PCR as well as going in for dissociation curves at the end of the run.

CYP1A1 and CYP1B1 quantitation across the panel of 20 NHMECs and MCF-7 cells for all treatment groups was carried out using 'Taqman chemistry'. The expression analysis across the 20 NHMECs (on treatment with BP in the absence or presence of CHL as detailed in Table 3) and 2 NHMECs (on treatment with $\mathrm{BP} / \mathrm{B}[\mathrm{e}] \mathrm{P}$ in the absence or presence of $\mathrm{CHL}$ as detailed in Table 4) were carried out on the ABI7700 Sequence Detection System (PE Applied Biosystems, Foster City, CA). CYP1A1 and CYP1B1 quantitation in MCF-7 cells and M00012 across different CHL concentrations (as detailed in Table 5) and in MCF-7 cells across different CHL treatments (as detailed in Table 3) was carried out on the ABI 7900HT Fast Real-Time PCR System (PE Applied Biosystems, Foster City, CA) using 'Taqman chemistry'. $1.0 \mu \mathrm{g}$ of total RNA (RNeasy kit; Qiagen, Valencia, CA) from each of the treated groups was used for cDNA synthesis. Single stranded cDNA (Advantage RT PCR kit; BD Biosciences, Palo Alto, CA) was used as template in a $50 \mu 1$ reaction mix consisting of $2 \mathrm{X}$ Taqman Universal PCR Master Mix and Taqman ${ }^{\circledR}$ Gene Expression Assay primers and probes for CYP1A1 (Hs00153120_ml) or CYPIBI (Hs00164383_ml) (Applied Biosystems, Foster City, CA). Each sample was assayed in duplicate and their expression normalized to that of GAPDH (Hs99999905_ml; Applied Biosystems, Foster City, CA) using the $2^{-\Delta \Delta C T}$ method (Livak et al. 2001). 


\subsection{Adduct analysis}

BP-DNA adducts formed on treatment of 20 NHMECs as well as MCF-7 cells with $\mathrm{BP}$ in the absence or presence of CHL was determined through a chemiluminiscence immunoassay (CIA). B[e]P-DNA adducts formed on treatment of 2 NHMEC strains with $\mathrm{B}[\mathrm{e}] \mathrm{P}$ in the absence or presence of CHL was analyzed through synchronous fluorescence spectrometry (SFS).

\subsubsection{DNA extraction}

DNA was extracted using a non-organic extraction protocol (Laird et al. 1991). Following treatment, cells were washed with 1X PBS, incubated in a lysis buffer $(100 \mathrm{mM}$ Tris $\mathrm{pH}$ 8.5, $5 \mathrm{mM}$ EDTA, $0.2 \% \mathrm{SDS}, 200 \mathrm{mM} \mathrm{NaCl}$ and $100 \mu \mathrm{g} / \mathrm{ml}$ Proteinase $\mathrm{K}$ ) at $37^{0} \mathrm{C}$ for $3 \mathrm{~h}$ and the DNA precipitated with an equal volume of isopropanol. The precipitated DNA was washed twice with $70 \%$ ethanol and then dissolved in nuclease free water.

\subsubsection{BP-DNA (BPdG) adduct analysis}

BPdG adducts were measured by a chemiluminiscence immunoassay (CIA) using antiserum elicited against DNA modified with $( \pm)-7 \beta, 8 \alpha$, dihydroxy $9 \alpha, 10 \alpha$-epoxy-7,8,9,10tetrahydrobenzo[ $\alpha]$ pyrene (BPDE) (8). Briefly, sonicated (20s, 20\% amplitude, Ultrasonic

Processor, Sonics \& Materials, Inc, Newton, CT) and heat denatured $\left(95^{\circ} \mathrm{C}, 4 \mathrm{~min}\right.$ and then cooled on ice) sample DNA or serially diluted standard BPDE-DNA (1.0 BPdG adduct/10 6 nucleotides) was mixed with an equal volume of rabbit anti-BPDE antiserum (1:3000000 in $0.25 \%$ casein prepared in phosphate buffered saline containing $0.05 \%$ Tween-20 (PBST)), incubated at $37^{\circ} \mathrm{C}$ for $15 \mathrm{~min}$ and added to wells of 96-well high binding LIA microtiter 
plates (Greiner Labortechnik, FRG) coated with highly modified BPDE-DNA or calf thymus DNA. These plates were prepared by coating with $100 \mathrm{pg}$ of highly modified, sonicated BPDE-DNA (0.33\% modified) or non-adducted calf thymus DNA in $0.1 \mathrm{ml}$ Reactibind solution (Pierce Biotechnology, Inc., Rockford, IL) for 48h following which they were washed with PBST containing $0.05 \%$ sodium azide and stored at $-20^{\circ} \mathrm{C}$ until used. The plates along with the sample or standard DNA were incubated at $37^{\circ} \mathrm{C}$ for $90 \mathrm{~min}$, washed with PBST, incubated with streptavidin alkaline phosphatase (Avidx-AP: 1:5000, 60 min; Applied Biosystems, Foster City, CA) prepared in $0.25 \%$ casein, washed again with PBST and Tris buffer (20 mM Tris, $1 \mathrm{mM} \mathrm{MgCl}$, $\mathrm{pH}$ 9.5) before finally incubating with CDP-Star solution containing Emerald II enhancer (Applied Biosystems, Foster City, CA). The plates were incubated overnight at $4^{0} \mathrm{C}$ and the luminescence measured at $542 \mathrm{~nm}$ on TR717 Microplate Luminometer (Applied Biosystems, Foster City, CA) on the following day after equilibration of the plates to room temperature. All samples were assayed in triplicate experimental wells and two separate CIAs.

\subsubsection{B[e]P-DNA adduct analysis}

DNA extracted using a non-organic protocol as detailed above were assayed for $\mathrm{B}[\mathrm{e}] \mathrm{P}-\mathrm{DNA}$ adducts using synchronous fluorescence spectrometry (SFS). $100 \mu \mathrm{g}$ of DNA was acid hydrolyzed $\left(90^{\circ} \mathrm{C}, 3 \mathrm{~h}\right)$, extracted three times with equal volumes of water-saturated isoamyl alcohol and then resuspended in $600 \mu \mathrm{l}$ HPLC grade water. Benzo(a)pyrene-r-7, t-8, c-9, t-10-tetrahydrotetrol (+/1) was used to generate a standard curve. Synchronous spectra were obtained by driving the excitation and emission monochromators simultaneously with a wavelength difference of $34 \mathrm{~nm}$. Fluorescent emission was obtained at emission wavelength 
of $380 \mathrm{~nm}$ (345 nm excitation wavelength). All measurements were carried out on a Perkin Elmer LS50B spectrophotometer (Perkin Elmer Corp., Rockville, MD) loaded with WinLab FL software.

\subsection{Statistical correlations}

Pearson correlation coefficients between the extent of expression of CYP1A1 and CYP1B1 on gene-chip (GC) versus RT-PCR as well as correlation coefficients between CYP1A1 and CYP1B1 expression and levels of carcinogen-DNA adducts on treatment of NHMECs or MCF-7 cells with BP or B[e]P in the absence or presence of CHL were determined using Excel (Saunders et al. 1994). Positive values indicate an increase in the value of the dependent variable with a corresponding increase in the independent variable and vice versa. 
Table 2: Details of different experiments carried out in the present study

\begin{tabular}{|c|c|c|c|}
\hline Experiment & $\begin{array}{l}\text { Number and } \\
\text { type of cell } \\
\text { strains used }\end{array}$ & Cell strains used & $\begin{array}{l}\text { Treatments } \\
\text { used }\end{array}$ \\
\hline $\begin{array}{l}\text { 1. Microarray } \\
\text { analysis }\end{array}$ & 6 NHMECs & $\begin{array}{l}\text { M98035, M99005, M98015, } \\
\text { M98025, M99025, M99016 }\end{array}$ & $\begin{array}{l}1,2 \text { and } 5 \text { as } \\
\text { detailed in } \\
\text { Table } 3\end{array}$ \\
\hline $\begin{array}{l}\text { 2. RT-PCR and DNA } \\
\text { adduct analysis }\end{array}$ & & & \\
\hline $\begin{array}{l}\text { a. On treatment with } \\
\mathrm{BP} \pm \mathrm{CHL}\end{array}$ & 20 NHMECs & $\begin{array}{l}\text { M98026, M98019, M98014, } \\
\text { M98011, M99021, M99006, } \\
\text { M99025, M0004, M98025, } \\
\text { M98035, M99005, M99003, } \\
\text { M00012, M99004, M98016, } \\
\text { M99015, M98030, M98015, } \\
\text { M98013, M99016 }\end{array}$ & $\begin{array}{l}1,2,3,4 \\
\text { and } 5 \text { as } \\
\text { detailed in } \\
\text { Table } 3\end{array}$ \\
\hline $\begin{array}{l}\text { b. On treatment with } \\
\mathrm{BP} \text { or } \mathrm{B}[\mathrm{e}] \mathrm{P} \pm \mathrm{CHL}\end{array}$ & 2 NHMECs & M98015 and M98025 & $\begin{array}{l}1,2,3,4 \\
\text { and } 5 \text { as } \\
\text { detailed in } \\
\text { Table } 4\end{array}$ \\
\hline $\begin{array}{l}\text { c. On comparison of } \\
\text { a NHMEC versus a } \\
\text { cancerous cell line } \\
\text { on treatment with BP } \\
\pm \text { CHL }\end{array}$ & $\begin{array}{l}20 \text { NHMECs } \\
\text { and MCF-7 }\end{array}$ & $\begin{array}{l}\text { The same } 20 \text { NHMECs } \\
\text { mentioned above and MCF-7 } \\
\text { (cancerous cell line) }\end{array}$ & $\begin{array}{l}1,2,3,4 \\
\text { and } 5 \text { as } \\
\text { detailed in } \\
\text { Table } 3\end{array}$ \\
\hline $\begin{array}{l}\text { d. On treatment with } \\
\mathrm{BP} \pm \text { a range of } \mathrm{CHL} \\
\text { concentrations }\end{array}$ & $\begin{array}{l}1 \text { NHMEC } \\
\text { strain and } \\
\text { MCF-7 }\end{array}$ & $\begin{array}{l}\text { M00012 (NHMEC strain) } \\
\text { and MCF-7 (cancerous cell } \\
\text { line) }\end{array}$ & $\begin{array}{l}1,2,3,4,5, \\
6 \text { and } 7 \text { as } \\
\text { detailed in } \\
\text { Table } 5\end{array}$ \\
\hline
\end{tabular}


Table 3: NHMECs and MCF-7 cells treatment protocol

\begin{tabular}{|c|c|c|}
\hline Treatment & & Treatment protocol \\
\hline $\begin{array}{l}\text { 1. Control vehicle } \\
\text { (SC) }\end{array}$ & - & Acetone: ethanol (1:1000)-24h \\
\hline 2. BP alone $(\mathbf{B P})$ & - & $\mathrm{BP}(4 \mu \mathrm{M})-24 \mathrm{~h}$ \\
\hline $\begin{array}{l}\text { 3. } \mathrm{CHL}+\mathrm{BP} \\
\text { (co-treatment) }\end{array}$ & - & {$[\mathrm{CHL}(5 \mu \mathrm{M})+\mathrm{BP}(4 \mu \mathrm{M})]-24 \mathrm{~h}$} \\
\hline $\begin{array}{l}\text { 4. Pre } \mathrm{CHL}+\text { post } \\
\text { BP treatment }\end{array}$ & CHL $(5 \mu \mathrm{M})-24 \mathrm{~h}$ & $\mathrm{BP}(4 \mu \mathrm{M})-24 \mathrm{~h}$ \\
\hline $\begin{array}{l}\text { 5. Pre } \mathrm{CHL}+\text { post } \\
\text { co-treatment }\end{array}$ & CHL $(5 \mu \mathrm{M})-24 \mathrm{~h}$ & {$[\mathrm{CHL}(5 \mu \mathrm{M})+\mathrm{BP}(4 \mu \mathrm{M})]-24 \mathrm{~h}$} \\
\hline
\end{tabular}


Table 4: Treatment protocol for the comparison of BP and B[e]P across 2 NHMECs (M98015 and M98025)

Treatment

1. Control vehicle (SC)

2. $\mathrm{BP}$ or $\mathrm{B}[\mathrm{e}] \mathrm{P}$ alone

3. $\mathrm{CHL}+\mathrm{BP}$ or $\mathrm{B}[\mathrm{e}] \mathrm{P}$ (co-treatment)

4. Pre $\mathrm{CHL}+$ post $\mathrm{BP}$ or $\mathrm{B}[\mathrm{e}] \mathrm{P}$ treatment

5. Pre $\mathrm{CHL}+$ post $\mathrm{BP}$ co-treatment or post CHL $(5 \mu \mathrm{M})-24 \mathrm{~h}$ $+$ $\mu \mathrm{M})]-24 \mathrm{~h}$

$\mu \mathrm{M})]-24 \mathrm{~h}$

\section{Treatment protocol}

Acetone: ethanol (1:1000)-24h

$\mathrm{BP}$ or B[e]P $(4 \mu \mathrm{M})-24 \mathrm{~h}$

$[\mathrm{CHL}(5 \mu \mathrm{M})+\mathrm{BP}$ or $\mathrm{B}[\mathrm{e}] \mathrm{P}(4$

CHL $(5 \mu \mathrm{M})-24 \mathrm{~h}+\mathrm{BP}$ or B[e]P $(4 \mu \mathrm{M})-24 \mathrm{~h}$

$\mathrm{B}[\mathrm{e}] \mathrm{P}$ co-treatment 
Table 5: Treatment protocol for the CHL dose response study comparing MCF-7 and an NHMEC (M00012) across a range of CHL concentrations

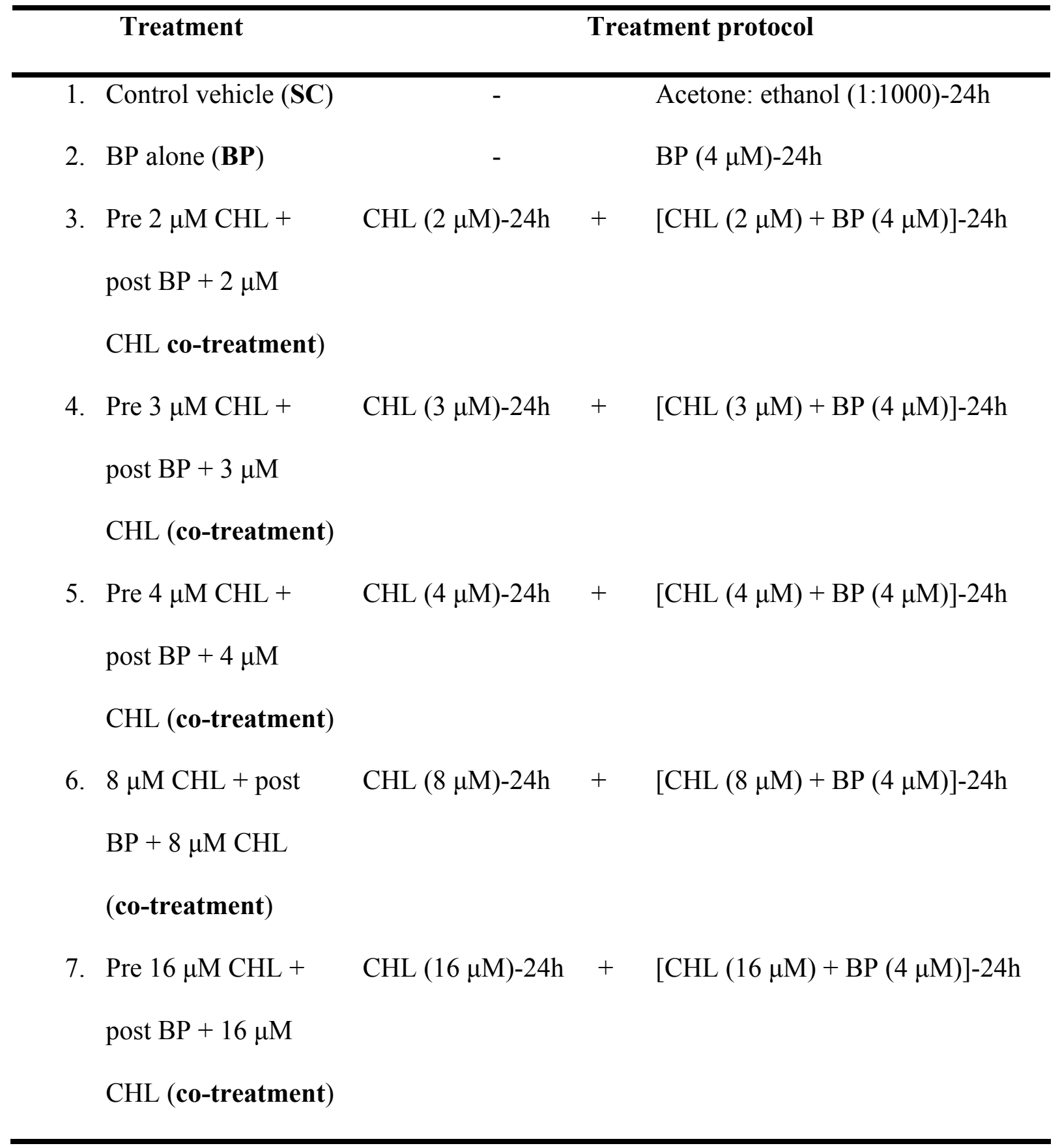




\subsection{RESULTS}

\subsection{Gene expression by microarray}

The expression of a multitude of genes involved in diverse cellular functions was altered by BP. The expression patterns of all the 22,283 probe sets (14,500 genes) on $\mathrm{Hu}-$ Gene 133A array in each of the 6 NHMEC strains on treatment with BP alone or in the presence of CHL (pre CHL + post co-treatment) are presented in Figure 7. The figure depicts a plot of the signal from each of the 22,283 probe sets for each of the respective treatment replicates versus the corresponding FC for those probe sets. It provides an overview of the scatter of expression patterns across cell strains and treatments. Each cell strain exhibited similar expression patterns for some of the probe sets yet unique expression patterns for others. The scatter plot also shows that for some probe sets while the signal may be low the overall FC compared to SC could be high and vice versa. While the expression of majority of the genes across most cell strains exhibited variation within 2-3 fold, there clearly appeared to be outliers which were treatment as well as cell strain specific. Of these probe sets, only those altered by a FC of $\sim 3.0$ (SLR $\geq 1.5$ ) in both the replicates upon comparison analysis of a respective treatment versus SC were considered altered. A complete list of all genes altered by SLR $\geq 1.5$ in at least one of the 6 NHMECs on comparison of $\mathrm{BP}$ treated as well as pre $\mathrm{CHL}+$ post co-treated cells versus $\mathrm{SC}$ can be found in Tables A, B, C , D and E at (http://www.cdc.gov/niosh/ext-suppmat/MTP/index.htm). Genes of the biotin synthesis pathway, bioB, bioC, bioD and the bacteriophage gene, cre spiked in the hybridized cocktail as hybridization controls were all detected on the arrays with signals in increasing order, as expected. Human GAPDH and Actin, house keeping genes were also detected. The average background, noise, percentage 
BP versus control vehicle
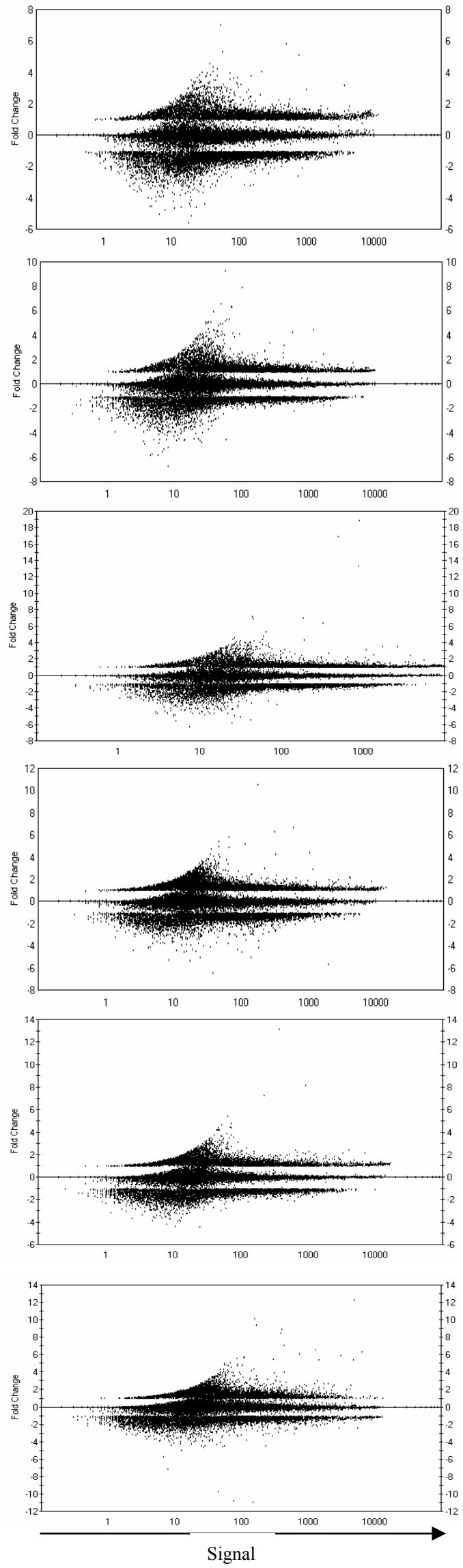

Pre CHL + co-treated versus control vehicle

M98035

M99005

M98015
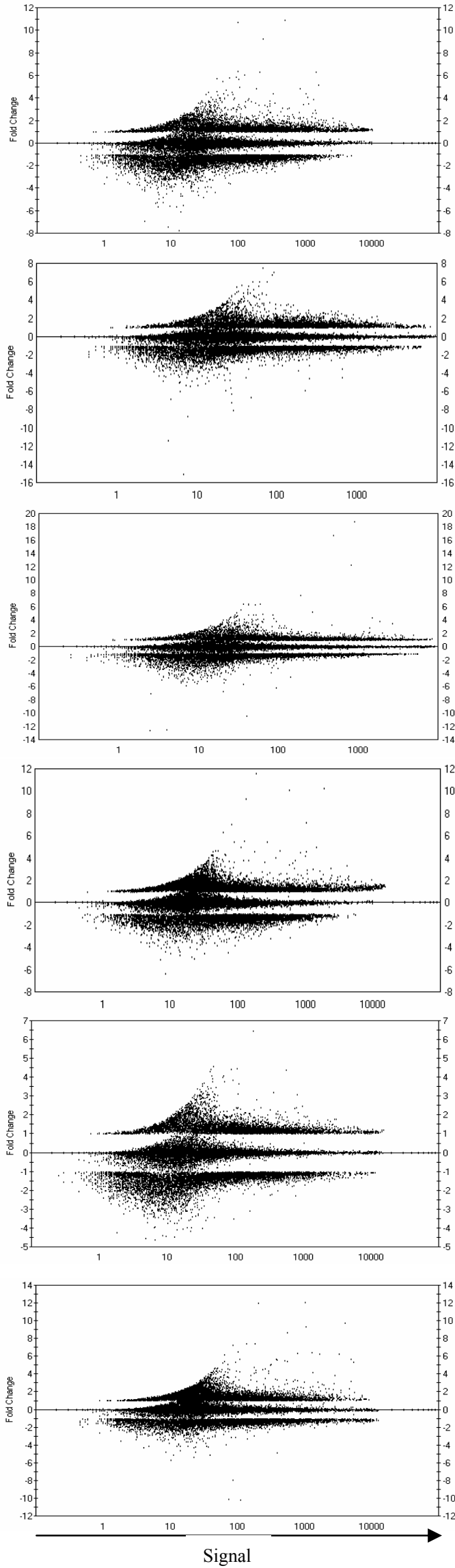

Figure 7: 'Fold change' scatter plots showing expression patterns of all the 22,283 probe sets on HuGene133A arrays on comparison of treated groups versus control vehicle. More details in the text. 
of genes present, absent and average signal intensities were also examined for each of the arrays.

A total of 49 genes $(0.38 \%)$ were altered in at least one of the 6 NHMEC strains on comparison of BP treated cells with SC when analyzed by MAS 5.0. Of these 43 were upregulated and only 6 were down-regulated. Only CYP1B1 exhibited consistent upregulation by SLR $\geq 1.5$ in all 6 cell strains. $A L D H 1 A 3$ (probe ID 222168_at) showed upregulation in 4 cell strains. All other genes were up-regulated in less than 4 cell strains. All down-regulated genes were from only one cell strain, M99016 and no gene was consistently down-regulated by SLR $\geq 1.5$ in all the cell strains. ILIB and SECTM1 were immune response genes consistently up-regulated by BP in most individuals with M99016 exhibiting the greatest expression with $\mathrm{SLR} \geq 1.5$. Both the above genes also exhibited the greatest expression in M99016 on pre-treatment with CHL followed by co-treatment and were, therefore, represented in the list of genes up-regulated by BP as well as pre CHL + post co-treatment. Overall, genes altered included those involved with xenobiotic metabolism, cell signaling, cell motility, cell proliferation, cellular transcription, metabolism, immune responses and other processes.

A total of 125 genes $(0.86 \%)$ were altered in at least one of the 6 NHMEC strains on comparison of pre CHL + post co-treated cells with SC when analyzed by MAS 5.0. Of these 103 were up-regulated and 22 down-regulated. Again, only CYP1B1 was consistently up-regulated by SLR $\geq 1.5$ in all but one cell strain. No gene was consistently downregulated by SLR $\geq 1.5$ in all cell strains. Gene categories modulated included mainly those altered by BP treatment in addition to genes involved in cell cycle control, apoptosis, DNA repair and various other cellular processes such as neurogenesis, gene regulation, and 
hormonal metabolism. A graphical display of all the altered genes generated using Cluster and Tree View (http://rana.lbl.gov/EisenSoftware.htm) according to the average linkage method (Sokal et al. 1958) is presented in Figure 8.

As per DMT 3.0 analysis a total of 5 different expression clusters were obtained on clustering of genes altered by comparison of treated groups versus SC (Figure 9). One cluster each was generated on clustering of genes significantly up-regulated and downregulated by BP as well as genes significantly down-regulated by pre treatment with $\mathrm{CHL}$ followed by co-treatment. However, two different expression clusters were generated for genes significantly up-regulated by pre $\mathrm{CHL}+$ post co-treatment. In all cases, the correlation of the altered genes belonging to the respective cluster pattern was 0.99 . A complete list of all the genes following the respective cluster patterns can be found in Tables G, H, I and J at (http://www.cdc.gov/niosh/ext-supp-mat/MTP/index.htm). More details of the characteristics of the genes used for the 'correlation coefficient' clustering are displayed in Table 6 .

Given the importance of immune responses in parallel with other cellular mechanisms such as detoxication and repair mechanisms to combat toxic stress, immune response genes altered by SLR $\geq 1.5$ in at least one of the 6 NHMECs in both the replicates upon comparison analysis of a respective treatment versus SC by MAS 5.0 were analyzed separately. Any interactions and cross-talks between the significantly altered immune response genes as well as other cellular proteins were analyzed. A total of only 5 immune response genes were altered by SLR $\geq 1.5$ on comparison of BP treated cells versus SC of which 4 were up-regulated and only 1 was down-regulated by SLR $\geq 1.5$ in at least one of the 6 NHMEC strains. The expression of 24 genes was altered by the synergistic 


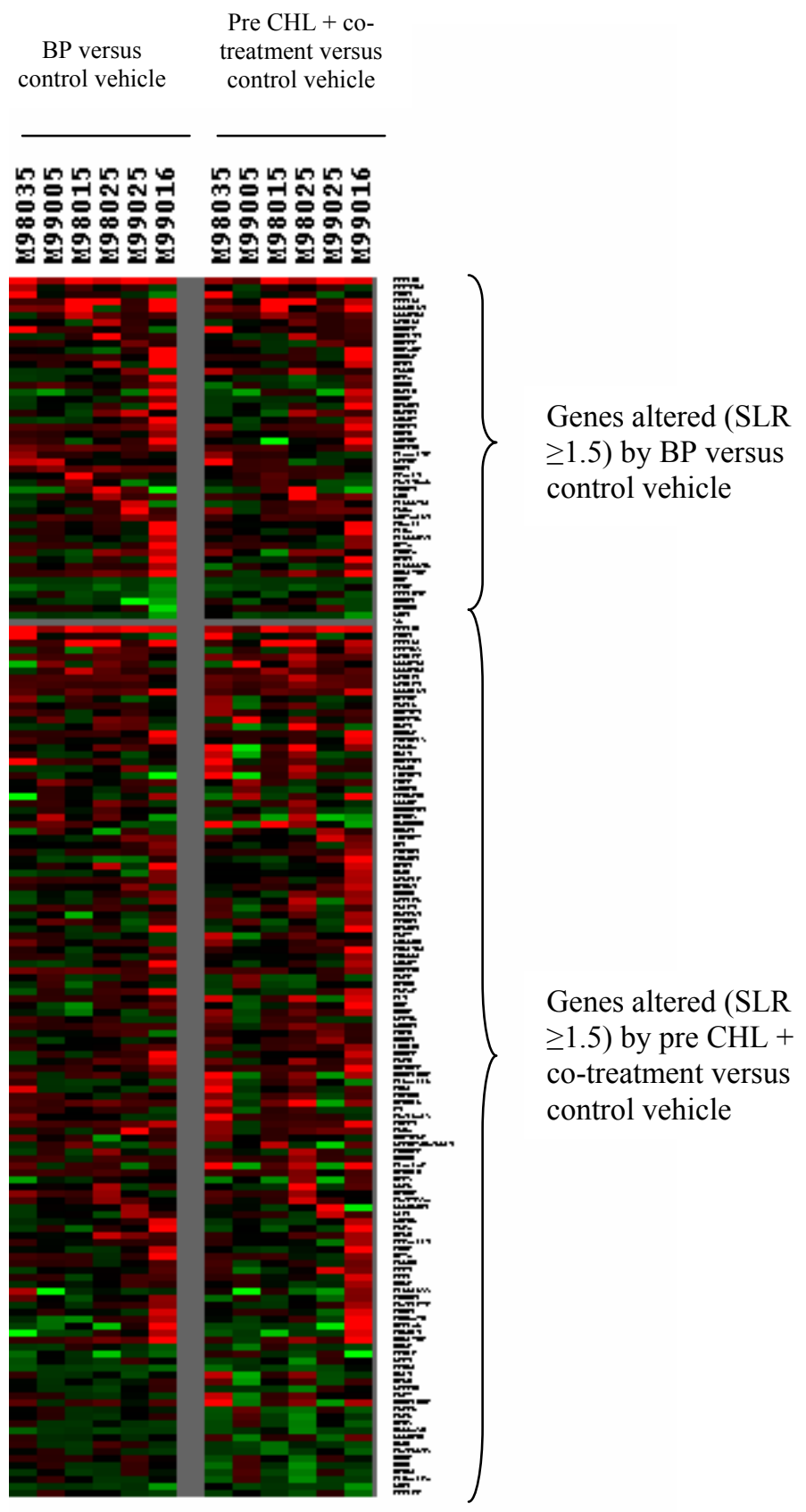

Figure 8: Graphical display (generated using Cluster and Tree View software) of all genes altered by SLR $\geq 1.5$ on Hu-Gene 133A arrays in at least one of the 6 NHMECs on treatment of cells with BP or pre CHL + co-treatment. A total of 49 genes were altered (up or down regulated) on treatment with $\mathrm{BP}$ alone and 125 on pre treatment with CHL followed by co-treatment. Though the gene names are not decipherable in the figure above due to the number of genes involved, a complete listing of all the above genes along with their corresponding FCs can be found at (http://www.cdc.gov/niosh/ext-suppmat/MTP/index.htm) [Tables A, B, C, D]. 


\section{Genes altered by BP treatment}

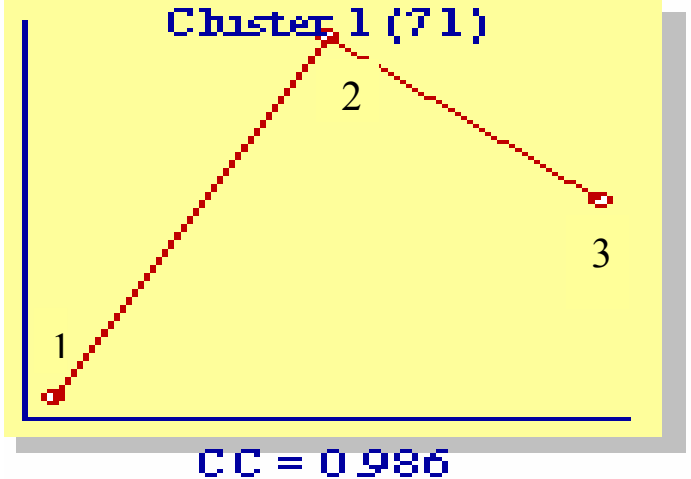

Cluster 1: BP versus SC - up-regulated genes

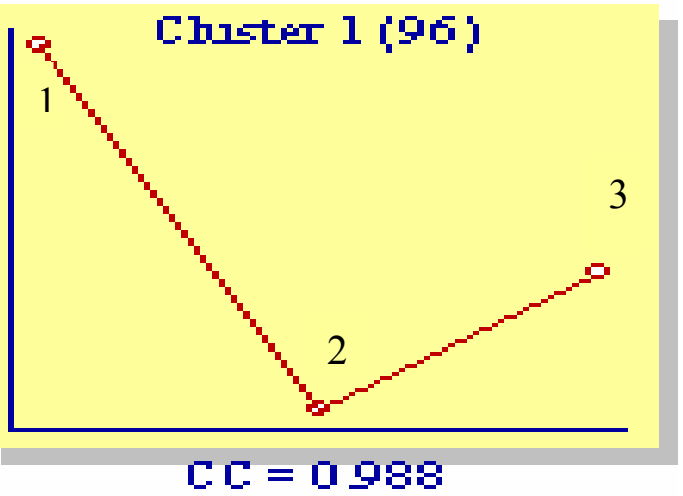

Cluster 2: BP versus SC - down-regulated genes

\section{Genes altered by Pre CHL + co-treatment}
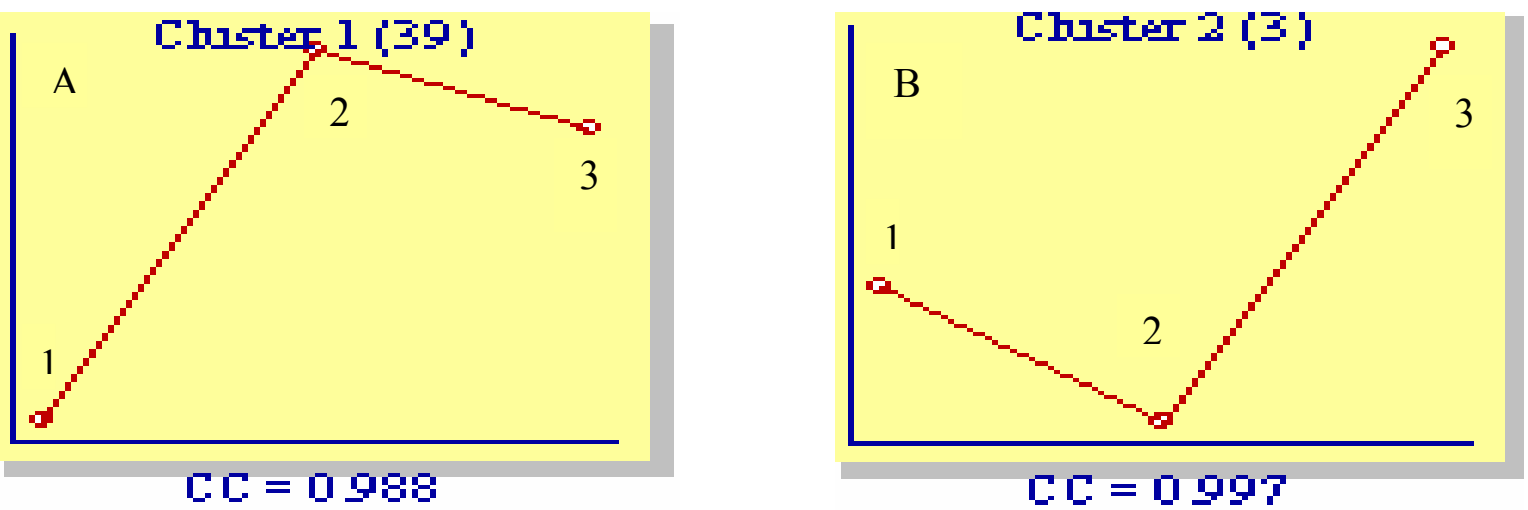

Clusters 3(A) and 3(B): Pre CHL + co-treatment versus SC - up-regulated genes

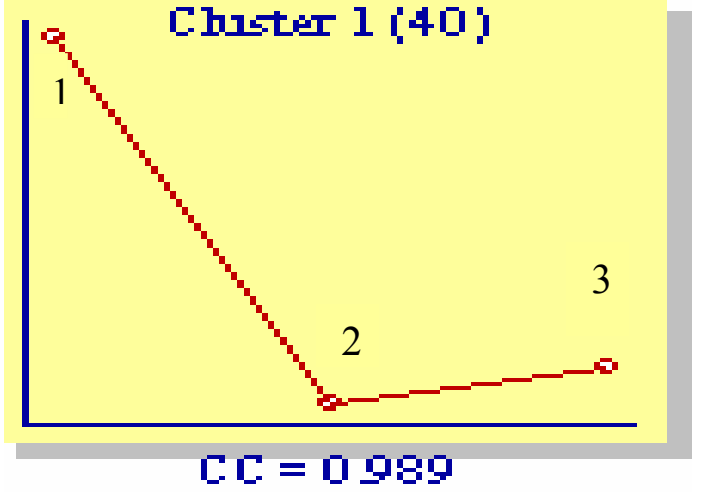

Cluster 4: Pre CHL + co-treatment versus $\mathrm{SC}$ - down-regulated genes
Figure 9: Correlation coefficient gene-expression clusters created from genes altered significantly in all 6 donors on comparison of the respective treated groups versus solvent control (SC). Each point on each cluster was calculated using the average signal generated by the respective treatment: $\mathbf{1}=\mathbf{S C}, \mathbf{2}=\mathbf{B P}$ alone and $\mathbf{3}=$ pre CHL + co-treatment. The number of genes/probes displaying the respective cluster pattern are shown in parenthesis besides each cluster. The correlation coefficient (CC) of the genes following the respective cluster pattern is shown below each cluster. Details about the exact genes in each cluster following the respective cluster pattern can be found at (http://www.cdc.gov/niosh/ext-suppmat/MTP/index.htm) [Tables G, H, I and J]. 
Table 6: Table representing the number and characteristics of the probe sets used to generate the clusters displayed in Figure 9

\begin{tabular}{lcccc}
\hline \multicolumn{1}{c}{ Comparison } & $\begin{array}{c}\text { Number of } \\
\text { probes common } \\
\text { to all 6 NHMECs } \\
\text { altered by } \\
\text { respective } \\
\text { comparison }\end{array}$ & $\begin{array}{c}\text { Number of probes } \\
\text { common to all } 6 \\
\text { NHMECs altered } \\
\text { significantly }(\mathrm{p}< \\
\text { 0.05) by the } \\
\text { respective } \\
\text { comparison }\end{array}$ & $\begin{array}{c}\text { Number of } \\
\text { significantly } \\
\text { altered probes }(\mathrm{p}< \\
\text { 0.05) exhibiting } \\
\text { common clustering } \\
\text { pattern }\end{array}$ & $\begin{array}{c}\text { Correlation } \\
\text { coefficient } \\
\text { of probes } \\
\text { belonging } \\
\text { to } \\
\text { respective } \\
\text { cluster }\end{array}$ \\
\hline $\begin{array}{l}\text { BP versus control vehicle } \\
\text { (up-regulated) }\end{array}$ & 391 & 106 & 71 & 0.99 \\
$\begin{array}{l}\text { BP versus control vehicle } \\
\text { (down-regulated) }\end{array}$ & 383 & 134 & 96 & 0.93 \\
$\begin{array}{l}\text { Pre CHL + co- treatment } \\
\text { versus control vehicle } \\
\text { (up-regulated) }\end{array}$ & 228 & 46 & 39,3 & $0.99,0.99$ \\
$\begin{array}{l}\text { Pre CHL + co- treatment } \\
\text { versus control vehicle } \\
\text { (down-regulated) }\end{array}$ & 232 & 41 & & \\
\hline
\end{tabular}


interaction of $\mathrm{BP}$ and $\mathrm{CHL}$ coming together in a pre $\mathrm{CHL}+$ co-treatment of which 15 genes were up-regulated and 9 were down-regulated by SLR $\geq 1.5$. The expression of $I L 1 B$ and SECTM1 were consistently up-regulated by BP in most individuals with M99016 exhibiting the greatest expression with $\mathrm{SLR} \geq 1.5$. Both genes also exhibited the greatest expression in M99016 on pre-treatment with CHL followed by co-treatment. A graphical display of all altered immune genes was also generated using Cluster and Tree View according to the average linkage method (Sokal et al. 1958) and is presented in Figure 10. Altered immune response genes were analyzed for cross-talks/signal transduction between themselves and with other cellular entities as well as other cellular pathways. Though the altered genes are fed as gene names to Pathway Studio, the software takes them as corresponding proteins while constructing interactions between the input entities as well as all other entities within the cell. When genes altered by BP treatment versus SC were queried for all possible interactions with all other molecules and processes within the cell using Pathway Studio, a total of 2248 relationships/interactions involving 1485 entities including proteins, small molecules, and various functional classes of molecules, complexes and cellular processes were found to be involved. When only 'shortest crosstalk' interactions were considered for the above genes only 60 relationships were found between 249 entities. However, there were no direct interactions between the 5 altered genes (Figure 11). More details about the various interactions among the immune response genes altered by comparison of $\mathrm{BP}$ versus $\mathrm{SC}$ can be found at (http://www.cdc.gov/niosh/ext-supp-mat/IR_Genes/index.htm) (IR-BP genes). 


\begin{tabular}{ll}
$\begin{array}{l}\text { BP versus } \\
\text { control } \\
\text { vehicle }\end{array}$ & $\begin{array}{l}\text { Pre CHL + co } \\
\text { versus control } \\
\text { vehicle }\end{array}$ \\
\hline
\end{tabular}

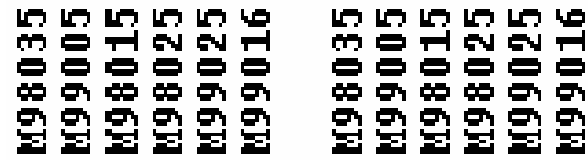

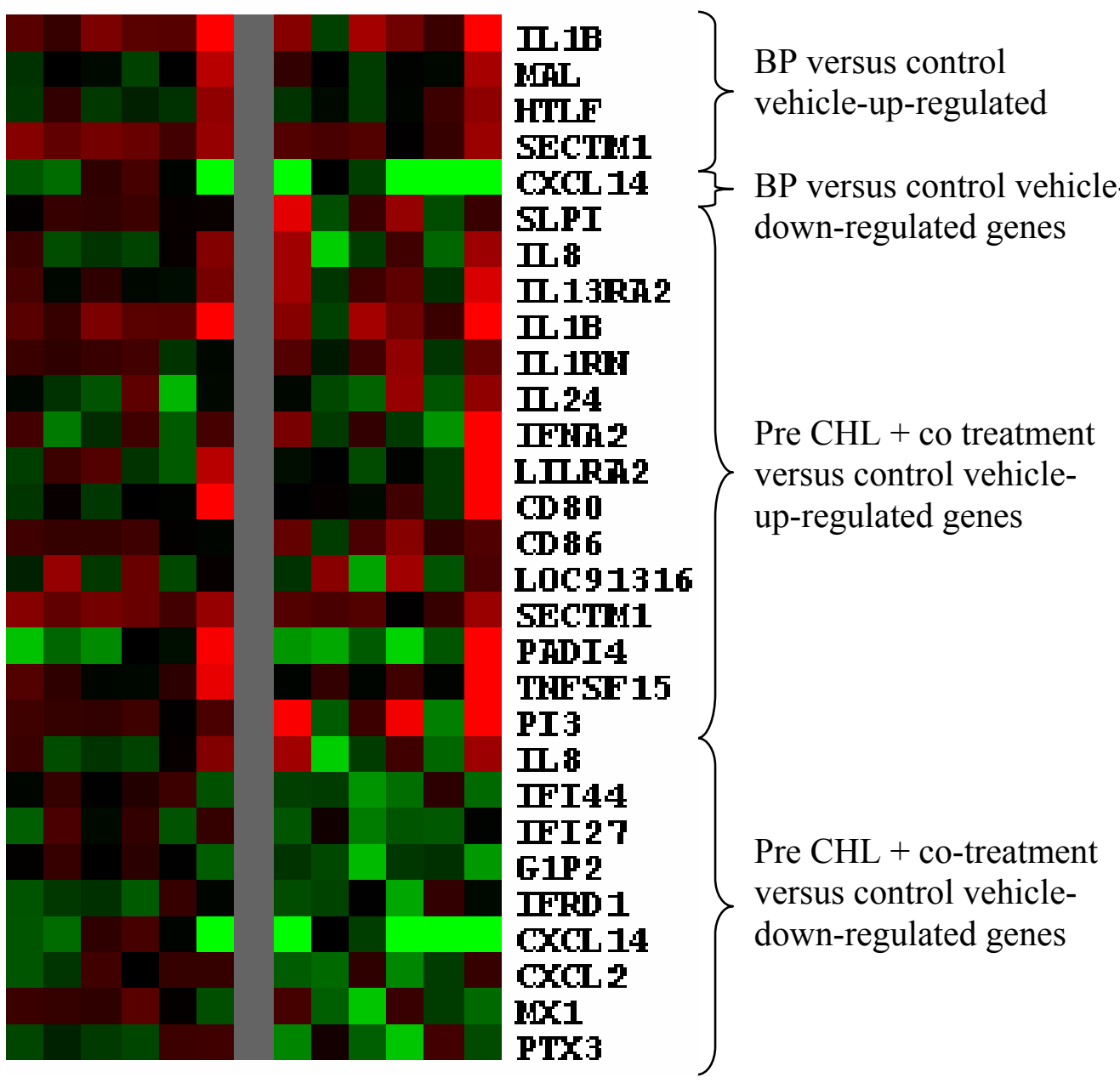

Figure 10: Graphical representation (generated using Cluster and Tree View software) of all immune response genes altered by SLR $\geq 1.5$ on Hu-Gene 133A arrays in at least one of the 6 NHMECs on treatment with BP or pre CHL + co-treatment. A complete listing of all the above immune response genes along with their corresponding FCs can be found at (http://www.cdc.gov/niosh/ext-supp-mat/IR_Genes/index.htm) [IR genes with FC table] 


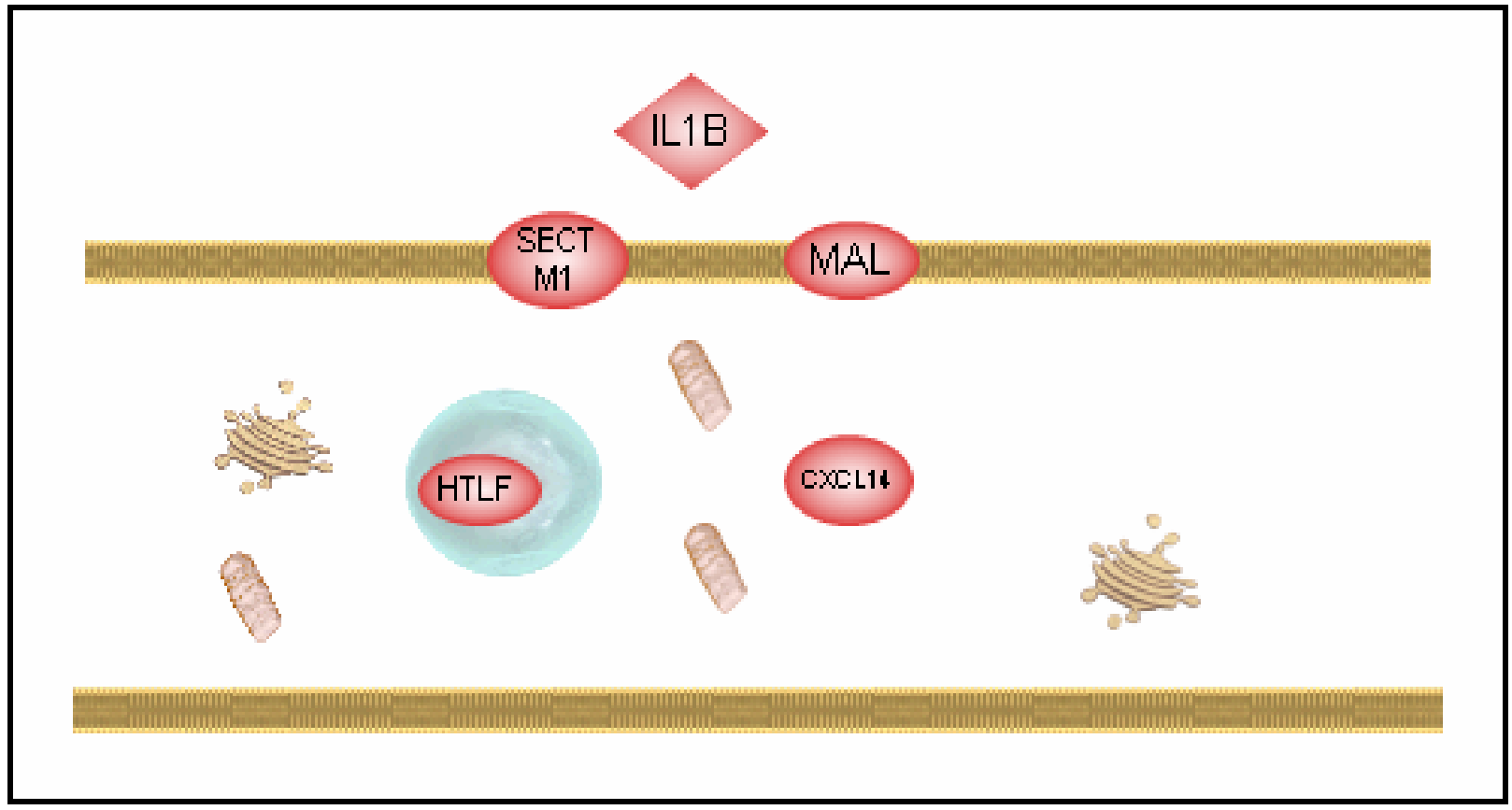

Figure 11: Five immune response genes were altered by SLR $\geq 1.5$ on $\mathrm{Hu}-\mathrm{Gene} 133 \mathrm{~A}$ arrays in at least one of the 6 NHMECs on exposure to BP alone. These five immune response genes were queried for any direct interactions between themselves using Pathway Studio software. The software takes the input genes as the corresponding proteins while trying to construct any interactions between the respective members. In this case, no direct interactions were observed between any of the input entities. The different immune response proteins are shown according to their respective cellular location. 
Genes altered by pre CHL + post co-treatment were found to exhibit 5782 interactions among and between 2299 entities within the cell. Of these 379 entities including the altered genes were found to be involved in 704 'shortest cross-talk' interactions. However, there were only 51 direct interactions between the altered genes (Figure 12). More details about the various interactions among the immune response genes altered by comparison of pre CHL + post co-treatment can be found at (http://www.cdc.gov/niosh/ext-supp-mat/IR_Genes/index.htm) (IR-CHL genes). It must be mentioned that since Pathway Studio relies largely on PubMed to construct interactions and since the content of PubMed is highly 'dynamic', the nature and number of interactions between the same input members and all other cellular entities are likely to vary with time.

Using ArrayXPath software, 2 (ILIB and MAL) out of the 5 genes altered by BP were found to have a statistically significant association $(\mathrm{p}<0.05)$ with 5 Biocarta pathways (Table 7). A total of 45 pathways in GenMAPP, 9 pathways in PharmGKB, 70 pathways in KEGG pathways and 346 Biocarta pathways were queried by Array X to obtain the above associations. A total of 2 (CD80 and CD86) out of 23 genes altered by pre $\mathrm{CHL}+$ post co-treatment were involved in one GenMAPP pathway and 6 genes (SLP1, $I L 8, I L 1 B, I L 1 R N, C D 80$ and $C D 86)$ were found to be involved in 13 Biocarta pathways (Table 8). 


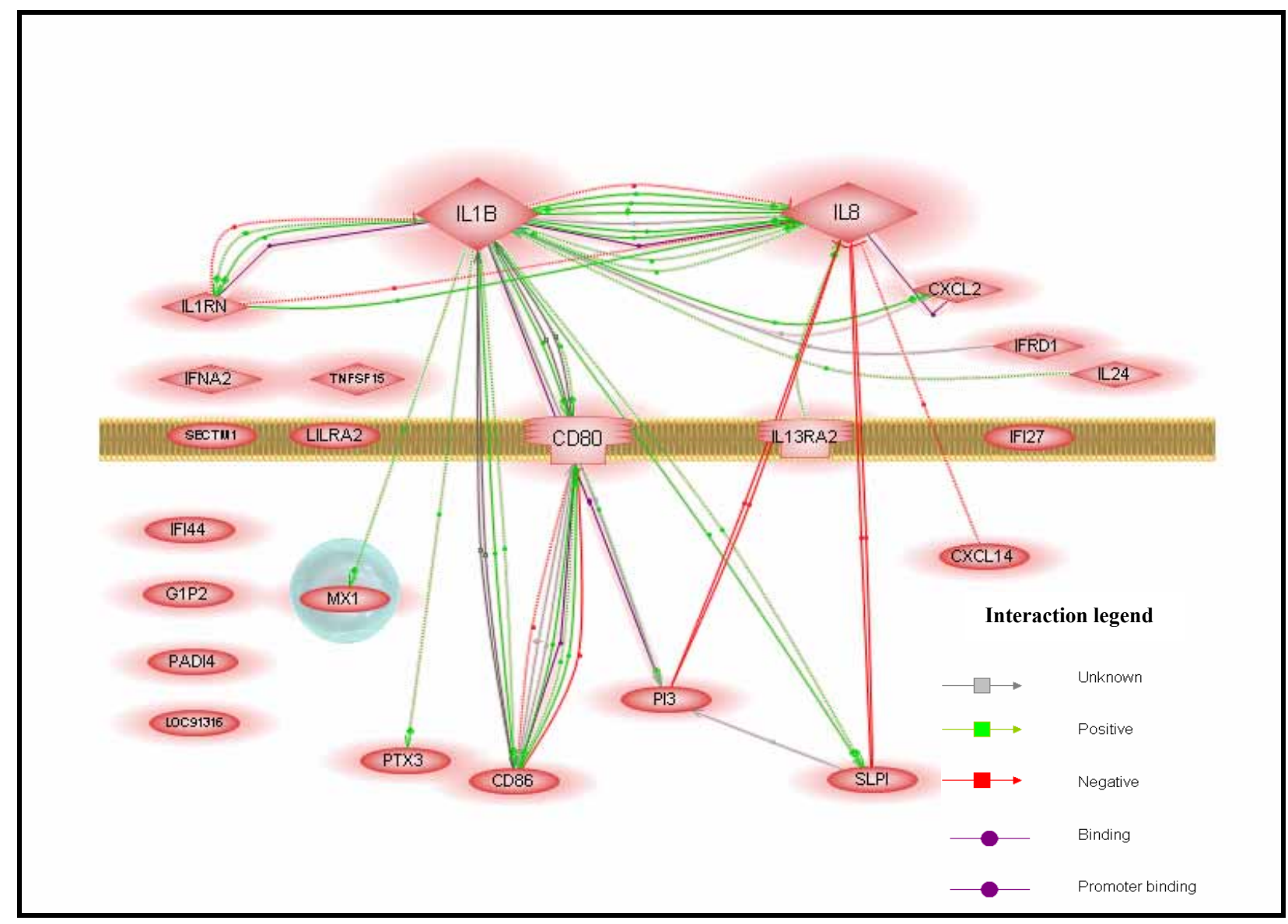

Figure 12: Twenty four immune response genes were altered by SLR $\geq 1.5$ on $\mathrm{Hu}-\mathrm{Gene}$ $133 \mathrm{~A}$ arrays in at least one of the 6 NHMECs on treatment with pre CHL + co-treatment. IL8 was up-regulated by SLR $\geq 1.5$ in some of the NHMECs but down-regulated by SLR $\geq$ 1.5 in the others and therefore represented in common in list of up-regulated and downregulated genes. These twenty four immune response genes were queried for any direct interactions between themselves using Pathway Studio software. The software takes the input genes as the corresponding proteins while trying to construct any interactions between the respective members. Fifty one direct interactions were observed between the input entities. The different immune response proteins are shown according to their respective cellular location. Genes with no direct interactions between themselves are shown as separate entities. 
Table 7: Pathways in which immune response (IR) genes altered by SLR $\geq 1.5$ on HuGene133A arrays on comparison of BP treated cells versus control vehicle are involved. A total of 5 IR genes were altered by SLR $\geq 1.5$ of which $2(40 \%)(I L 1 B, M A L)$ were found to possess a statistically significant association $(\mathrm{p} \leq 0.05)$ with the above pathways. For genes involved in more than one pathway, a $q$ value ( $q \leq 0.05$, equivalent to $p$ value) accounts for the multiple-comparison.

\begin{tabular}{|l|c|c|c|}
\hline Pathway & $\begin{array}{c}\text { Identified } \\
\text { node }\end{array}$ & p-value & q-value \\
\hline \hline BioCarta//Hs_IL 5 Signaling Pathway & $1 / 8(19)$ & 0.0101 & 0.0154 \\
\hline \hline $\begin{array}{l}\text { BioCarta//Hs_NFkB activation by Nontypeable Hemophilus } \\
\text { influenzae }\end{array}$ & $1 / 24(43)$ & 0.0300 & 0.0230 \\
\hline \hline BioCarta//Hs_Signal transduction through IL1R & $1 / 30(41)$ & 0.0374 & 0.0230 \\
\hline \hline BioCarta//Hs_Role of MAL in Rho-Mediated Activation of SRF & $1 / 19(26)$ & 0.0238 & 0.0230 \\
\hline \hline BioCarta//Hs_Msp/Ron Receptor Signaling Pathway & $1 / 6(27)$ & 0.0075 & 0.0232 \\
\hline
\end{tabular}


Table 8: Pathways in which immune response (IR) genes altered by SLR $\geq 1.5$ on $\mathrm{Hu}-$ Gene133A arrays on comparison of CHL treated cells (pre CHL + co-treated) versus control vehicle are involved. A total of 23 IR genes were altered by SLR $\geq 1.5$ of which 6 (26\%) (SLP1 IL8,IL1B,IL1RN, CD80 and CD86) were found to possess a statistically significant association $(\mathrm{p} \leq 0.05)$ with the above pathways. For genes involved in more than one pathway, a $q$ value ( $q \leq 0.05$, equivalent to $p$ value) accounts for the multiplecomparison.

\begin{tabular}{|l|c|c|c|}
\hline Pathway & $\begin{array}{c}\text { Identified } \\
\text { node }\end{array}$ & p-value & q-value \\
\hline $\begin{array}{l}\text { BioCarta//Hs_Proepithelin Conversion to Epithelin and Wound } \\
\text { Repair Control }\end{array}$ & $2 / 6(40)$ & 0.0002 & 0.0018 \\
\hline $\begin{array}{l}\text { BioCarta//Hs_The Co-Stimulatory Signal During T-cell } \\
\text { Activation }\end{array}$ & $2 / 16(40)$ & 0.0014 & 0.0072 \\
\hline $\begin{array}{l}\text { BioCarta//Hs_NFkB activation by Nontypeable Hemophilus } \\
\text { influenzae }\end{array}$ & $2 / 24(43)$ & 0.0032 & 0.0110 \\
\hline BioCarta//Hs_Signal transduction through IL1R & $2 / 30(41)$ & 0.0049 & 0.0128 \\
\hline BioCarta//Hs_Free Radical Induced Apoptosis & $1 / 10(11)$ & 0.0373 & 0.0430 \\
\hline BioCarta//Hs_IL_5 Signaling Pathway & $1 / 8(19)$ & 0.0299 & 0.0430 \\
\hline BioCarta//Hs_Msp/Ron Receptor Signaling Pathway & $1 / 6(27)$ & 0.0225 & 0.0430 \\
\hline \hline BioCarta//Hs_B Lymphocyte Cell Surface Molecules & $1 / 9(10)$ & 0.0336 & 0.0430 \\
\hline \hline BioCarta//Hs_Antigen Dependent B Cell Activation & $1 / 10(24)$ & 0.0373 & 0.0430 \\
\hline \hline BioCarta//Hs_IL_17 Signaling Pathway & $1 / 15(16)$ & 0.0554 & 0.0443 \\
\hline BioCarta//Hs_Regulation of hematopoiesis by cytokines & $1 / 15(41)$ & 0.0554 & 0.0443 \\
\hline BioCarta//Hs_Adhesion and Diapedesis of Lymphocytes & $1 / 14(47)$ & 0.0518 & 0.0443 \\
\hline \hline BioCarta//Hs_Adhesion and Diapedesis of Granulocytes & $1 / 15(49)$ & 0.0554 & 0.0443 \\
\hline \hline BioCarta//Hs_Cells and Molecules involved in local acute \\
inflammatory response
\end{tabular}




\subsection{RT-PCR}

\subsubsection{Confirmation of gene expression on microarrays}

Confirmation of gene expression patterns on microarrays was carried out through RT-PCR for a selected subset 24 genes altered by SLR $\geq 1.5$ in both replicates in at least one of the 6 NHMECs. Of these, 7 were immune response genes. The results of RT-PCR confirmation are presented in Tables 9A, 9B and 10. A more extensive version of Tables 9A and 9B can be found in Table E and Table F, respectively at (http://www.cdc.gov/niosh/ext-supp-mat/MTP/index.htm). For genes altered by BP, there was a very good correlation of FC as measured by GC and RT-PCR for all genes except for CALB1 and SECTM1 which exhibited only a moderate correlation and MERTK which exhibited a very poor correlation. A very good correlation of FC between GC and RT-PCR was also observed for all genes altered on pre-treatment with CHL followed by cotreatment except for $A K R 1 C 2, D H R S 3$ and MCM5 which exhibited only a moderate correlation.

As for the immune response genes there was a good correlation for some genes while the remaining correlated moderately to poorly (Table 10). Upon BP treatment there was a good correlation for $1 L 1 B$ and $C X C L 2$, a moderate correlation for $P I 3$ but a poor correlation for the remaining genes (SECTM1, IL1RN, CD86 and MX1). However, more genes had a better correlation on pre $\mathrm{CHL}+$ co-treatment. ILIRN and PI3 also exhibited a good correlation of expression on GC versus RT-PCR apart from $1 L 1 B$ and CXCL2 while CD86 and $M X 1$ exhibited a moderate correlation. 
Table 9A: Correlation of fold change (FC) on Hu-Gene 133A arrays/gene-chip (GC) and RT-PCR for a partial list of genes altered by SLR $\geq 1.5$ in at least one of the 6 NHMECs when treated with BP alone. A more extensive version of this table can be found at (http://www.cdc.gov/niosh/ext-supp-mat/MTP/index.htm) [Table E].

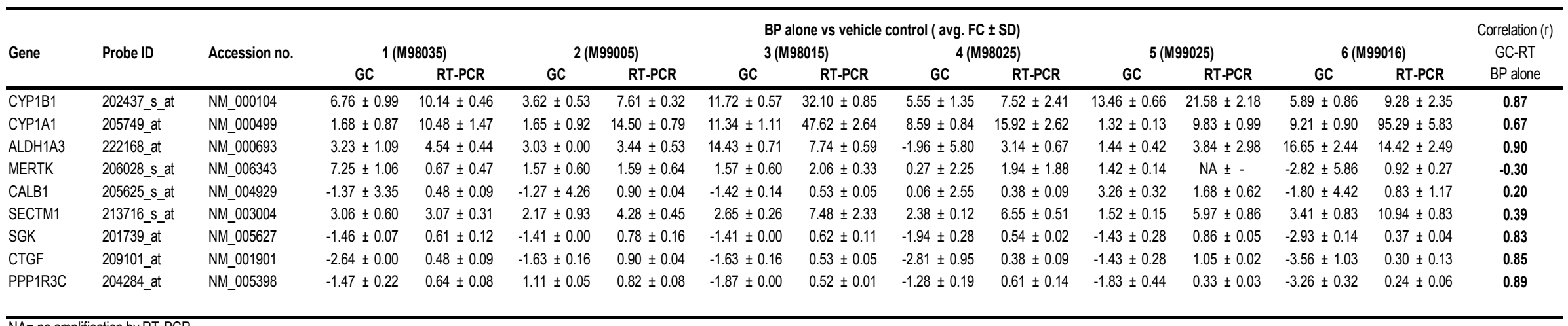

$=$ only 5 donors with amplification considered for correlation analysis

Table 9B: Correlation of fold change (FC) on Hu-Gene 133A arrays/gene-chip (GC) and RT-PCR for a partial list of genes altered by SLR $\geq 1.5$ in at least one of the 6 NHMECs when treated with pre-CHL followed by co-treatment. A more extensive version of this table can be found at (http://www.cdc.gov/niosh/ext-supp-mat/MTP/index.htm) [Table F].

\begin{tabular}{|c|c|c|c|c|c|c|c|c|c|c|c|c|c|c|c|}
\hline \multirow{3}{*}{ Gene } & \multirow{3}{*}{ Probe ID } & \multirow{3}{*}{ Accession no. } & \multicolumn{12}{|c|}{ Pre CHL + co-treatment vs vehicle control ( avg. $\mathrm{FC} \pm \mathrm{SD}$ ) } & \multirow{3}{*}{$\begin{array}{c}\text { Correlation (r) } \\
\text { GC-RT } \\
\text { PreCHL + co }\end{array}$} \\
\hline & & & \multicolumn{2}{|c|}{1 (M98035) } & \multicolumn{2}{|c|}{2 (M99005) } & \multicolumn{2}{|c|}{3 (M98015) } & \multicolumn{2}{|c|}{4 (M98025) } & \multicolumn{2}{|c|}{5 (M99025) } & \multicolumn{2}{|c|}{6 (M99016) } & \\
\hline & & & GC & RT-PCR & GC & RT-PCR & GC & RT-PCR & GC & RT-PCR & GC & RT-PCR & GC & RT-PCR & \\
\hline AKR1C2 & 211653 x_at & NM_001354 & $3.04 \pm 0.30$ & $1.62 \pm 0.13$ & $1.74 \pm 0.00$ & $1.82 \pm 0.06$ & $2.07 \pm 0.10$ & $2.00 \pm 0.16$ & $5.10 \pm 0.25$ & $2.39 \pm 0.08$ & $2.08 \pm 0.30$ & $2.25 \pm 0.22$ & $2.22 \pm 0.11$ & $1.76 \pm 0.48$ & 0.50 \\
\hline CLDN4 & 201428_at & NM_001305 & $7.03 \pm 1.37$ & $4.87 \pm 0.39$ & $-6.06 \pm 0.00$ & $0.13 \pm 0.01$ & $1.88 \pm 0.37$ & $1.43 \pm 0.08$ & $15.13 \pm 6.46$ & $3.71 \pm 0.14$ & $-0.31 \pm 2.59$ & $0.46 \pm 0.04$ & $3.62 \pm 0.53$ & $0.35 \pm 0.04$ & 0.76 \\
\hline DHRS3 & 202481_at & NM_004753 & $1.80 \pm 0.09$ & $1.52 \pm 0.17$ & $-1.19 \pm 0.06$ & $0.96 \pm 0.03$ & $1.76 \pm 0.34$ & $2.17 \pm 0.94$ & $3.26 \pm 0.32$ & $1.53 \pm 0.16$ & $2.30 \pm 0.00$ & $3.35 \pm 0.28$ & $1.15 \pm 0.00$ & $1.52 \pm 0.16$ & 0.50 \\
\hline SOD2 & 216841_s_at & NM_000636 & $-1.15 \pm 0.00$ & $0.66 \pm 0.02$ & $-3.14 \pm 0.15$ & $0.38 \pm 0.06$ & $1.15 \pm 0.00$ & $1.28 \pm 0.07$ & $-1.33 \pm 0.26$ & $0.51 \pm 0.05$ & $-1.15 \pm 0.11$ & $0.87 \pm 0.08$ & $-1.81 \pm 0.69$ & $0.11 \pm 0.02$ & 0.83 \\
\hline CYR61 & 201289_at & NM_001554 & $-2.83 \pm 0.00$ & $0.23 \pm 0.01$ & $-1.11 \pm 0.05$ & $1.00 \pm 0.05$ & $-1.62 \pm 0.00$ & $0.53 \pm 0.02$ & $-2.30 \pm 0.00$ & $0.27 \pm 0.02$ & $-1.80 \pm 0.09$ & $0.62 \pm 0.05$ & $-0.22 \pm 1.83$ & $0.97 \pm 0.15$ & 0.92 \\
\hline SERPINB2 & 204614_at & NM_002575 & $9.52 \pm 0.47$ & $10.18 \pm 1.55$ & $-3.14 \pm 0.15$ & $0.25 \pm 0.01$ & $1.46 \pm 0.07$ & $1.75 \pm 0.14$ & $3.49 \pm 0.34$ & $2.20 \pm 0.28$ & $-4.00 \pm 0.00$ & $0.25 \pm 0.01$ & $4.46 \pm 0.65$ & $0.58 \pm 0.03$ & 0.81 \\
\hline MCM5 & 216237_s_at & NM_006739 & $-1.87 \pm 0.18$ & $0.33 \pm 0.02$ & $1.69 \pm 0.25$ & $1.84 \pm 0.52$ & $-1.52 \pm 0.15$ & $0.49 \pm 0.02$ & $-3.26 \pm 0.32$ & $0.23 \pm 0.02$ & $-0.07 \pm 1.52$ & $1.34 \pm 0.32$ & $-2.35 \pm 0.68$ & $1.08 \pm 0.22$ & 0.37 \\
\hline GADD45B & 207574_s_at & NM_015675 & $-1.87 \pm 0.18$ & $0.30 \pm 0.03$ & $-1.23 \pm 0.12$ & $0.69 \pm 0.07$ & $-0.28 \pm 1.91$ & $0.79 \pm 0.09$ & $-3.38 \pm 0.50$ & $0.16 \pm 0.03$ & $-1.57 \pm 0.08$ & $0.83 \pm 0.15$ & $-1.37 \pm 0.20$ & $0.27 \pm 0.02$ & 0.71 \\
\hline
\end{tabular}

A negative sign for FC by gene-chip (GC) refers to down-regulation of gene- expression by the corresponding value. 
Table 10: Correlation of FC on gene-chip (GC) versus RT-PCR for a selected set of immune response genes altered by SLR $\geq 1.5$ in at least one of the 6 NHMECs on treatment with $\mathrm{BP}$ in the absence or presence of $\mathrm{CHL}$

\begin{tabular}{|c|c|c|c|c|c|c|c|c|c|}
\hline \multirow{3}{*}{ Gene } & \multirow{3}{*}{ Cell strain } & \multicolumn{8}{|c|}{ Treatment } \\
\hline & & \multicolumn{3}{|c|}{$\mathrm{BP}$ versus control vehicle $\mathrm{FC}$} & \multirow{2}{*}{$\begin{array}{c}\text { Correlation ( } r) \\
\text { FC by GC vs RT }\end{array}$} & \multicolumn{3}{|c|}{ Pre $\mathrm{CHL}+$ co versus control vehicle $\mathrm{FC}$} & \multirow{2}{*}{$\frac{\text { Correlation }(r)}{\text { FC by GC vs RT }}$} \\
\hline & & & $\overline{G C}$ & RT-PCR & & & $\overline{G C}$ & RT-PCR & \\
\hline \multirow{6}{*}{ IL1B } & M98035 & 2.00 & \pm 0.20 & $3.68 \pm 0.56$ & \multirow{6}{*}{0.97} & 3.03 & \pm 0.00 & $3.47 \pm 3.03$ & \multirow{6}{*}{0.88} \\
\hline & M99005 & 1.23 & \pm 0.00 & $1.68 \pm 0.05$ & & -1.46 & \pm 0.07 & $0.62 \pm-1.46$ & \\
\hline & M98015 & 2.83 & \pm 0.00 & $5.21 \pm 0.73$ & & 3.73 & \pm 0.00 & $5.61 \pm 3.73$ & \\
\hline & M98025 & 2.02 & \pm 0.39 & $1.29 \pm 0.05$ & & 2.55 & \pm 0.12 & $1.22 \pm 2.55$ & \\
\hline & M99025 & 1.94 & \pm 0.28 & $3.20 \pm 0.51$ & & 1.32 & \pm 0.00 & $1.41 \pm 1.32$ & \\
\hline & M99016 & 5.89 & \pm 0.86 & $11.52 \pm 1.20$ & & 5.89 & \pm 0.86 & $7.44 \pm 5.89$ & \\
\hline \multirow{6}{*}{ SECTM1 } & M98035 & 3.06 & \pm 0.60 & $2.87 \pm 0.34$ & \multirow{6}{*}{0.33} & 1.87 & \pm 0.00 & $0.65 \pm 0.09$ & \multirow{6}{*}{0.43} \\
\hline & M99005 & 2.17 & \pm 0.93 & $5.17 \pm 1.15$ & & 1.64 & \pm 0.32 & $1.83 \pm 0.29$ & \\
\hline & M98015 & 2.65 & \pm 0.26 & $9.22 \pm 1.19$ & & 1.88 & \pm 0.37 & $4.17 \pm 0.53$ & \\
\hline & M98025 & 2.38 & \pm 0.12 & $4.87 \pm 0.96$ & & 0.05 & \pm 1.93 & $2.07 \pm 0.70$ & \\
\hline & M99025 & 1.52 & \pm 0.15 & $4.84 \pm 0.77$ & & 1.23 & \pm 0.00 & $1.67 \pm 0.18$ & \\
\hline & M99016 & 3.41 & \pm 0.83 & $8.76 \pm 0.56$ & & 3.49 & \pm 0.34 & $3.60 \pm 0.50$ & \\
\hline \multirow{6}{*}{ IL1RN } & M98035 & 1.32 & \pm 0.00 & $1.60 \pm 0.13$ & \multirow{6}{*}{0.12} & 1.87 & \pm 0.00 & $1.33 \pm 0.05$ & \multirow{6}{*}{0.76} \\
\hline & M99005 & 1.04 & \pm 0.05 & $1.59 \pm 0.16$ & & -0.53 & \pm 1.55 & $0.53 \pm 0.01$ & \\
\hline & M98015 & 1.28 & \pm 0.06 & $1.77 \pm 0.15$ & & 1.52 & \pm 0.15 & $1.47 \pm 0.06$ & \\
\hline & M98025 & 1.52 & \pm 0.00 & $0.93 \pm 0.06$ & & 3.26 & \pm 0.32 & $1.39 \pm 0.08$ & \\
\hline & M99025 & -1.15 & \pm 0.00 & $1.39 \pm 0.16$ & & -1.19 & \pm 0.06 & $1.01 \pm 0.10$ & \\
\hline & M99016 & -0.17 & \pm 1.76 & $1.23 \pm 0.44$ & & 2.25 & \pm 0.55 & $1.76 \pm 0.36$ & \\
\hline \multirow{6}{*}{ CD86 } & M98035 & 1.41 & \pm 0.00 & $1.66 \pm 0.11$ & \multirow{6}{*}{0.28} & 2.25 & \pm 0.55 & $1.64 \pm 0.17$ & \multirow{6}{*}{0.64} \\
\hline & M99005 & 1.19 & \pm 0.06 & $1.91 \pm 0.11$ & & -1.37 & \pm 0.07 & $0.73 \pm 0.03$ & \\
\hline & M98015 & 1.21 & \pm 0.29 & $1.57 \pm 0.17$ & & 1.68 & \pm 0.08 & $2.28 \pm 0.21$ & \\
\hline & M98025 & 1.41 & \pm 0.00 & $1.17 \pm 0.25$ & & 3.04 & \pm 0.30 & $1.62 \pm 0.09$ & \\
\hline & M99025 & 0.09 & \pm 1.75 & $1.94 \pm 0.16$ & & 1.15 & \pm 0.11 & $1.13 \pm 0.14$ & \\
\hline & M99016 & -0.12 & \pm 1.69 & $0.78 \pm 0.25$ & & 1.83 & \pm 0.44 & $2.40 \pm 1.05$ & \\
\hline \multirow{6}{*}{$\mathrm{PI} 3$} & M98035 & 2.82 & \pm 0.60 & $1.43 \pm 0.28$ & \multirow{6}{*}{0.41} & 7.51 & \pm 0.87 & $5.63 \pm 1.89$ & \multirow{6}{*}{0.86} \\
\hline & M99005 & 1.54 & \pm 0.07 & $1.19 \pm 0.06$ & & 0.48 & \pm 0.10 & $-2.08 \pm 0.30$ & \\
\hline & M98015 & 1.29 & \pm 0.09 & $1.11 \pm 0.05$ & & 1.40 & \pm 0.10 & $1.37 \pm 0.07$ & \\
\hline & M98025 & 1.03 & \pm 0.08 & $1.42 \pm 0.14$ & & 3.05 & \pm 0.17 & $5.47 \pm 0.27$ & \\
\hline & M99025 & 1.58 & \pm 0.97 & $0.05 \pm 2.07$ & & 0.36 & \pm 0.13 & $-2.87 \pm 1.22$ & \\
\hline & M99016 & 2.95 & \pm 0.38 & $1.69 \pm 0.25$ & & 5.44 & \pm 1.15 & $5.74 \pm 3.19$ & \\
\hline & M98035 & -1.93 & \pm 0.09 & $0.70 \pm 0.09$ & & -2.08 & \pm 0.30 & $0.26 \pm 0.04$ & \\
\hline & M99005 & -1.19 & \pm 0.06 & $1.62 \pm 0.16$ & & -2.38 & \pm 0.12 & $0.53 \pm 0.06$ & \\
\hline $\mathrm{CXCl} 2$ & M98015 & 1.46 & \pm 0.07 & $2.31 \pm 0.19$ & 079 & 1.04 & \pm 0.05 & $1.23 \pm 0.12$ & 0.77 \\
\hline CXCLL & M98025 & 0.00 & \pm 2.00 & $1.05 \pm 0.55$ & 0.19 & -3.04 & \pm 0.30 & $0.17 \pm 0.02$ & \\
\hline & M99025 & 1.19 & \pm 0.06 & $1.97 \pm 0.48$ & & -1.37 & \pm 0.20 & $0.75 \pm 0.04$ & \\
\hline & M99016 & 1.21 & \pm 0.29 & $1.67 \pm 0.29$ & & 1.23 & \pm 0.12 & $0.63 \pm 0.02$ & \\
\hline & M98035 & 1.32 & \pm 0.13 & $1.34 \pm 0.08$ & & 1.57 & \pm 0.60 & $0.55 \pm 0.12$ & \\
\hline & M99005 & 1.19 & \pm 0.06 & $1.85 \pm 0.37$ & & -2.14 & \pm 0.00 & $0.19 \pm 0.02$ & \\
\hline MX1 & M98015 & 1.07 & \pm 0.11 & $0.96 \pm 0.10$ & 0.16 & -4.46 & \pm 0.65 & $0.10 \pm 0.04$ & 0.53 \\
\hline IVIX1 & M98025 & 1.99 & \pm 0.67 & $1.14 \pm 0.10$ & 0.16 & 1.28 & \pm 0.19 & $0.16 \pm 0.04$ & 0.53 \\
\hline & M99025 & 0.12 & \pm 1.69 & $2.02 \pm 0.46$ & & -1.33 & \pm 0.12 & $0.51 \pm 0.36$ & \\
\hline & M99016 & -1.80 & \pm 0.09 & $0.93 \pm 0.32$ & & -2.39 & \pm 0.91 & $0.20 \pm 0.04$ & \\
\hline
\end{tabular}




\subsection{2 $C Y P 1 A 1$ and $C Y P 1 B 1$ expression in 20 NHMECs on treatment with BP in the absence or presence of CHL}

Wide inter-individual variations were observed both in the induction and modulation of CYP1A1 (Table 11) and CYPIBI (Table 12) following treatment of 20 NHMECs with BP in the absence or presence of CHL. The basal expression of CYP1A1 was highest in M98014 $(\triangle \mathrm{Ct}=$ 11.18) and lowest in $\mathrm{M} 99005(\Delta \mathrm{Ct}=16.61)$. The basal expression of $C Y P 1 B 1$ was highest in M99016 $(\Delta \mathrm{Ct}=4.19)$ and lowest in M98015 $(\Delta \mathrm{Ct}=9.77)$. Overall, the average basal expression of $C Y P 1 A$ in the 20 NHMECs $(\triangle \mathrm{Ct}=13.44)$ was 53 fold lower than that of $C Y P 1 B 1(\triangle \mathrm{Ct}=$ 7.72). The basal expression in terms of $\Delta \mathrm{Ct}$ for the $20 \mathrm{NHMECs}$ is presented in Table 13.

All CHL treatments caused mitigation of $C Y P 1 A 1$ and $C Y P 1 B 1$ expression in 18/20 cell strains (4-88\% reduction across the different CHL treatments) and 17/20 cell (4-83\% reduction across the different $\mathrm{CHL}$ treatments) strains respectively, compared to those treated with BP alone.

CHL co-treatment mitigated $C Y P 1 A 1$ and $C Y P 1 B 1$ expression in all 20 cell strains. CYP1A1 expression in the presence of CHL co-treatment ranged from 2-62 fold (4-75\% reduction compared to cells treated with $\mathrm{BP}$ alone) while that of $C Y P 1 B 1$ ranged from 1-36 fold (4-67\% reduction).

Pre $\mathrm{CHL}+$ post BP treatment enhanced BP induced $C Y P 1 A 1$ expression in 2/20 cell strains (3\% and 81\% in M98011 and M98014, respectively) while decreasing it in the remaining 18/20 cell strains (11-76\% reduction). CYP1B1 expression was also enhanced by pre $\mathrm{CHL}+$ post BP treatment in 3/20 cell strains (30\%, 20\% and 11\% in M98019, M98011 and M99006, respectively) while decreasing it in the remaining 17/20 cell strains (8-60\% reduction). 
Table 11: Inter-individual variations in the induction and modulation of CYP1A1 upon exposure of NHMECs to either BP alone or in combination with CHL

\begin{tabular}{lcccc}
\hline \multicolumn{5}{c}{ CYP1A1 } \\
\hline \multicolumn{5}{c}{ Treatment } \\
\hline Cell strain & \multicolumn{4}{c}{2} \\
M98019 & $3.62 \pm 1.04$ & $1.85^{*} \pm 0.53$ & $3.03 \pm 0.63$ & $2.38 \pm 0.83$ \\
M98014 & $4.57 \pm 1.52$ & $2.79 \pm 0.25$ & $8.25 \pm 1.91$ & $5.90 \pm 0.91$ \\
M98011 & $5.36 \pm 1.98$ & $5.12 \pm 0.99$ & $5.50 \pm 0.56$ & $4.99 \pm 2.63$ \\
M99021 & $5.90 \pm 1.46$ & $3.73 \pm 1.61$ & $3.75^{*} \pm 0.36$ & $3.59^{*} \pm 0.52$ \\
M99006 & $6.46 \pm 1.54$ & $4.21 \pm 1.01$ & $5.31 \pm 0.61$ & $3.49 \pm 0.80$ \\
M99025 & $6.58 \pm 2.94$ & $4.06 \pm 1.59$ & $2.62 \pm 0.48$ & $1.65^{*} \pm 0.46$ \\
M90004 & $7.07 \pm 2.53$ & $4.02 \pm 0.48$ & $4.35 \pm 0.81$ & $3.79 \pm 1.41$ \\
M98025 & $11.22 \pm 3.22$ & $6.01 * \pm 2.61$ & $9.76 \pm 1.62$ & $6.94 \pm 0.65$ \\
M98035 & $13.83 \pm 2.89$ & $12.82 \pm 2.01$ & $9.70 \pm 1.93$ & $5.29 * \pm 1.58$ \\
M99005 & $13.69 \pm 1.35$ & $7.89 * \pm 1.69$ & $8.14^{*} \pm 1.11$ & $4.11^{*} \pm 1.37$ \\
M99003 & $14.27 \pm 5.02$ & $10.21 \pm 2.29$ & $11.49 \pm 1.60$ & $13.02 \pm 3.96$ \\
M00012 & $17.09 \pm 2.89$ & $11.00 * \pm 1.72$ & $12.19^{*} \pm 2.80$ & $9.82^{*} \pm 2.68$ \\
M99004 & $20.25 \pm 2.06$ & $10.78^{*} \pm 3.96$ & $10.54 * \pm 3.42$ & $11.55^{*} \pm 1.58$ \\
M98016 & $32.50 \pm 3.40$ & $9.99^{*} \pm 2.25$ & $13.2 * \pm 1.35$ & $6.09^{*} \pm 2.02$ \\
M99015 & $35.88 \pm 4.71$ & $20.75^{*} \pm 3.02$ & $31.78 \pm 2.69$ & $29.91 \pm 9.71$ \\
M98030 & $44.32 \pm 12.41$ & $18.54^{*} \pm 1.53$ & $25.50^{*} \pm 2.09$ & $15.97^{*} \pm 4.05$ \\
M98015 & $49.95 \pm 6.64$ & $44.79 \pm 12.10$ & $42.59 \pm 10.52$ & $47.42 \pm 9.35$ \\
M98026 & $52.71 \pm 12.93$ & $38.99 \pm 10.16$ & $31.34^{*} \pm 11.54$ & $30.27^{*} \pm 5.99$ \\
M98013 & $66.72 \pm 12.10$ & $16.36^{*} \pm 6.40$ & $14.72^{*} \pm 6.90$ & $8.03^{*} \pm 4.95$ \\
M99016 & $95.84 \pm 9.86$ & $62.36^{*} \pm 20.75$ & $62.36^{*} \pm 4.82$ & $53.54^{*} \pm 5.38$ \\
\hline & & & & \\
\hline
\end{tabular}

Treatments - 1-BP alone, 2-co-treatment, 3-Pre CHL+ post BP treatment, 4-Pre CHL + co-treatment (Details of treatments in Materials and methods)

Numbers shown represent fold change \pm standard deviation $(n=2)$ as measured by RT-PCR

$*$ = statistically significant reduction in expression $(\mathrm{p} \leq 0.05)$ compared to expression induced by BP alone 
Table 12: Inter-individual variations in the induction and modulation of CYP1B1 upon exposure of NHMECs to either BP alone or in combination with CHL

\begin{tabular}{lcccc}
\hline \multicolumn{5}{c}{ CYP1B1 } \\
\hline \multicolumn{5}{c}{ Treatment } \\
\hline Cell strain & \multicolumn{4}{c}{2} \\
M98019 & $6.09 \pm 0.38$ & $4.40 \pm 1.95$ & $7.90 \pm 1.00$ & 4 \\
M98014 & $3.49 \pm 0.58$ & $2.92 \pm 0.90$ & $1.93^{*} \pm 0.18$ & $1.86^{*} \pm 0.25$ \\
M98011 & $4.74 \pm 0.86$ & $3.69 \pm 0.63$ & $5.69 \pm 0.90$ & $6.32 \pm 1.53$ \\
M99021 & $8.75 \pm 1.41$ & $5.78^{*} \pm 1.01$ & $6.27^{*} \pm 1.20$ & $5.46^{*} \pm 0.86$ \\
M99006 & $8.92 \pm 1.57$ & $5.54^{*} \pm 0.44$ & $9.88 \pm 3.36$ & $4.80^{*} \pm 0.76$ \\
M99025 & $22.86 \pm 3.92$ & $11.35 \pm 3.09$ & $9.19 \pm 1.15$ & $6.50 \pm 1.26$ \\
M90004 & $7.92 \pm 2.62$ & $5.10 \pm 0.54$ & $7.31 \pm 2.91$ & $4.53 \pm 1.95$ \\
M98025 & $8.41 \pm 2.23$ & $4.42^{*} \pm 1.81$ & $4.81^{*} \pm 1.50$ & $3.88^{*} \pm 0.54$ \\
M98035 & $9.46 \pm 1.89$ & $7.97 \pm 1.84$ & $7.31 \pm 1.75$ & $3.32 \pm 0.40$ \\
M99005 & $9.16 \pm 1.78$ & $7.48 \pm 0.42$ & $5.82^{*} \pm 1.65$ & $3.49^{*} \pm 1.30$ \\
M99003 & $19.66 \pm 1.86$ & $13.02^{*} \pm 1.22$ & $15.27^{*} \pm 0.97$ & $16.48 \pm 5.15$ \\
M00012 & $17.54 \pm 3.14$ & $10.32^{*} \pm 1.65$ & $14.67 \pm 4.43$ & $11.90^{*} \pm 3.29$ \\
M99004 & $12.13 \pm 1.25$ & $7.67^{*} \pm 1.34$ & $6.96^{*} \pm 0.72$ & $6.89^{*} \pm 0.83$ \\
M98016 & $14.65 \pm 2.98$ & $6.16^{*} \pm 0.61$ & $10.04 \pm 5.87$ & $3.86^{*} \pm 1.12$ \\
M99015 & $33.88 \pm 1.73$ & $13.50^{*} \pm 2.25$ & $22.59 \pm 11.19$ & $19.94^{*} \pm 3.73$ \\
M98030 & $16.00 \pm 4.12$ & $7.01^{*} \pm 1.35$ & $6.45^{*} \pm 0.99$ & $4.89^{*} \pm 1.77$ \\
M98015 & $43.41 \pm 4.80$ & $35.88^{*} \pm 3.30$ & $39.74^{*} \pm 9.32$ & $39.26 \pm 6.89$ \\
M98026 & $31.67 \pm 3.34$ & $21.08^{*} \pm 1.69$ & $24.21 \pm 8.40$ & $23.55 \pm 6.40$ \\
M98013 & $42.01 \pm 6.89$ & $13.95^{*} \pm 1.46$ & $11.49^{*} \pm 1.88$ & $7.00^{*} \pm 3.24$ \\
M99016 & $8.94 \pm 1.39$ & $8.60 \pm 1.61$ & $8.27 \pm 1.08$ & $6.92 \pm 1.26$ \\
\hline & & & & \\
\hline
\end{tabular}

Treatments - 1-BP alone, 2-co-treatment, 3-Pre CHL+ post BP treatment, 4-Pre CHL + co-treatment (Details of treatments in Materials and methods)

Numbers shown represent fold change \pm standard deviation $(\mathrm{n}=2)$ as measured by RT-PCR

$*=$ statistically significant reduction in expression $(\mathrm{p} \leq 0.05)$ compared to expression induced by BP alone 
Table 13: Basal $C Y P 1 A 1$ and $C Y P 1 B 1$ expression levels $(\triangle \mathrm{Ct})$ in the 20 NHMECs normalized to that of GAPDH

\begin{tabular}{lcc}
\hline \multicolumn{3}{c}{ Basal expression levels $(\Delta \mathbf{C t})$} \\
\hline Cell strain & CYP1A1 & CYP1B1 \\
\hline & & \\
M98019 & 15.39 & 8.67 \\
M98014 & 11.18 & 6.75 \\
M98011 & 12.92 & 7.80 \\
M99021 & 11.30 & 7.62 \\
M99006 & 12.26 & 8.32 \\
M99025 & 15.05 & 8.63 \\
M00004 & 12.26 & 7.03 \\
M98025 & 12.10 & 6.35 \\
M98035 & 15.05 & 7.48 \\
M99005 & 16.61 & 6.06 \\
M99003 & 13.48 & 8.70 \\
M00012 & 12.78 & 7.92 \\
M99004 & 12.46 & 7.65 \\
M98016 & 13.43 & 7.60 \\
M99015 & 12.34 & 9.29 \\
M98030 & 15.60 & 6.62 \\
M98015 & 12.18 & 9.77 \\
M98026 & 12.99 & 8.81 \\
M98013 & 13.93 & 8.84 \\
M99016 & 15.86 & 4.19 \\
& & \\
\hline Average & 13.46 & 7.71
\end{tabular}


Pre CHL + post co-treatment enhanced CYPlAl expression in $1 / 20$ cell strains $(29 \%$ in M98014) while decreasing it in the remaining 19/20 cell strains (5-88\% reduction). CYP1B1 expression was enhanced in 2/20 cell strains (11\% and 33\% in M98019 and M98011 respectively) while decreasing it in the remaining 18/20 cell strains (10-83\% reduction).

There was a poor correlation between $C Y P 1 A 1$ and $C Y P 1 B 1$ induction on treatment with BP alone $\left(r^{2}=0.28\right)$ and by pre $\mathrm{CHL}+$ post BP treatment $\left(\mathrm{r}^{2}=0.29\right)$ but a moderate correlation on co-treatment $\left(\mathrm{r}^{2}=0.41\right)$ and pre $\mathrm{CHL}+$ post co-treatment $\left(\mathrm{r}^{2}=0.47\right)($ Figure 13$)$. The correlation between the percentage modulation in the expression of CYPIA1 to that of CYPIBI on cotreatment as well as pre CHL + post co-treatment was moderate $\left(r^{2}=0.46,0.41\right.$, respectively $)$ and absent on treatment with pre $\mathrm{CHL}+$ post $\mathrm{BP}\left(\mathrm{r}^{2}=0.09\right)$ (Figure 14).

This was mainly due to the presence of several outliers in pre $\mathrm{CHL}+$ post $\mathrm{BP}$ treatment (M98011 and M98014 for CYP1A1 and M98019, M98011 and M99006 for CYP1B1) and pre CHL + post co-treatment groups (M98014 for CYP1A1 and M98019 and M98011 for CYP1B1). Removal of these outliers from the analysis significantly improved the correlation between the percentage modulation in the expression of $C Y P 1 A 1$ to that of $C Y P 1 B 1$ upon pre $\mathrm{CHL}+$ post cotreatment $\left(\mathrm{r}^{2}=0.73\right)$. However, the correlation between the modulation of CYPIAl to that of CYP1B1 on pre CHL + post BP treatment improved only marginally $\left(\mathrm{r}^{2}=0.34\right)$.

The correlation $\left(\mathrm{r}^{2}\right)$ between $C Y P 1 A 1$ expression by BP alone and in the presence of different CHL treatments was $0.78,0.78$ and 0.64 for co-treatment, pre CHL + post BP treatment and pre CHL + post co-treatment, respectively (Figure 15). The correlation $\left(\mathrm{r}^{2}\right)$ between $C Y P 1 B 1$ expression by BP alone and in the presence of different CHL treatments was $0.72,0.66$ and 0.57 for co-treatment, pre $\mathrm{CHL}+$ post $\mathrm{BP}$ and pre $\mathrm{CHL}+$ post cotreatment, respectively) (Figure 16). 

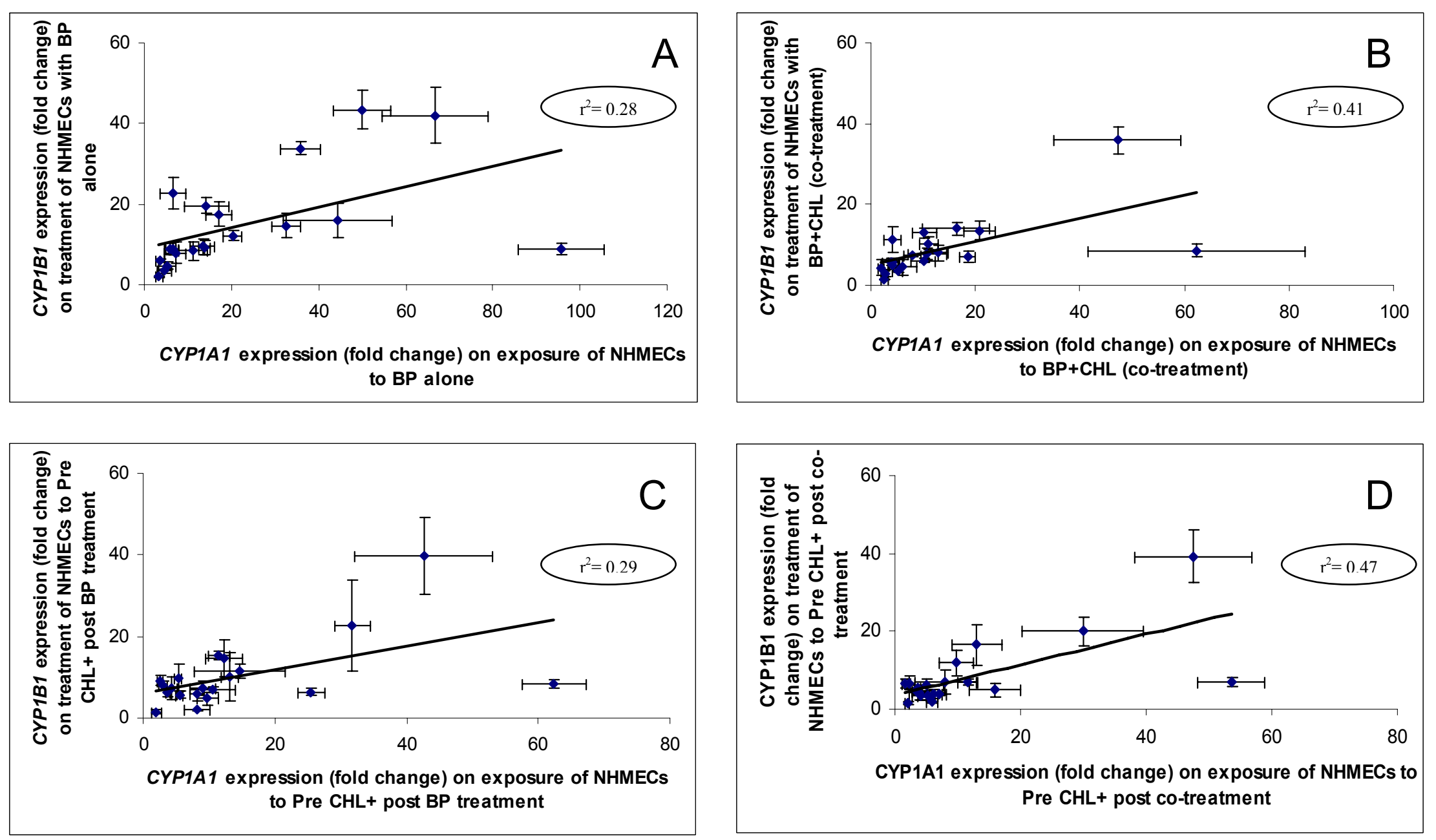

Figure 13: Correlation of $C Y P 1 A 1$ expression to that of $C Y P 1 B 1$ in the $20 \mathrm{NHMECs}$ 


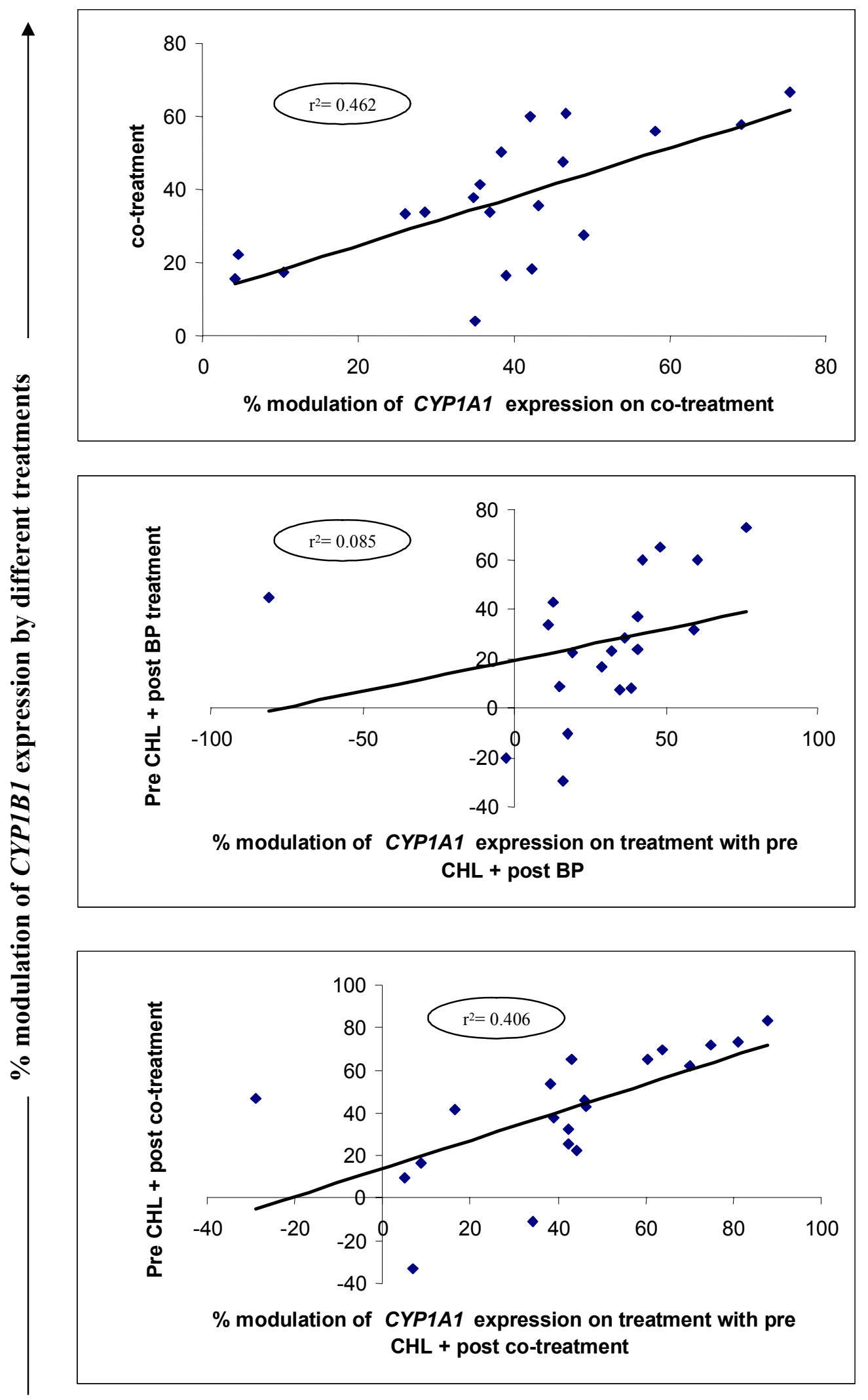

Figure 14: Correlation between the percentage modulation in the expression of CYP1A1 to that of CYP1B1 


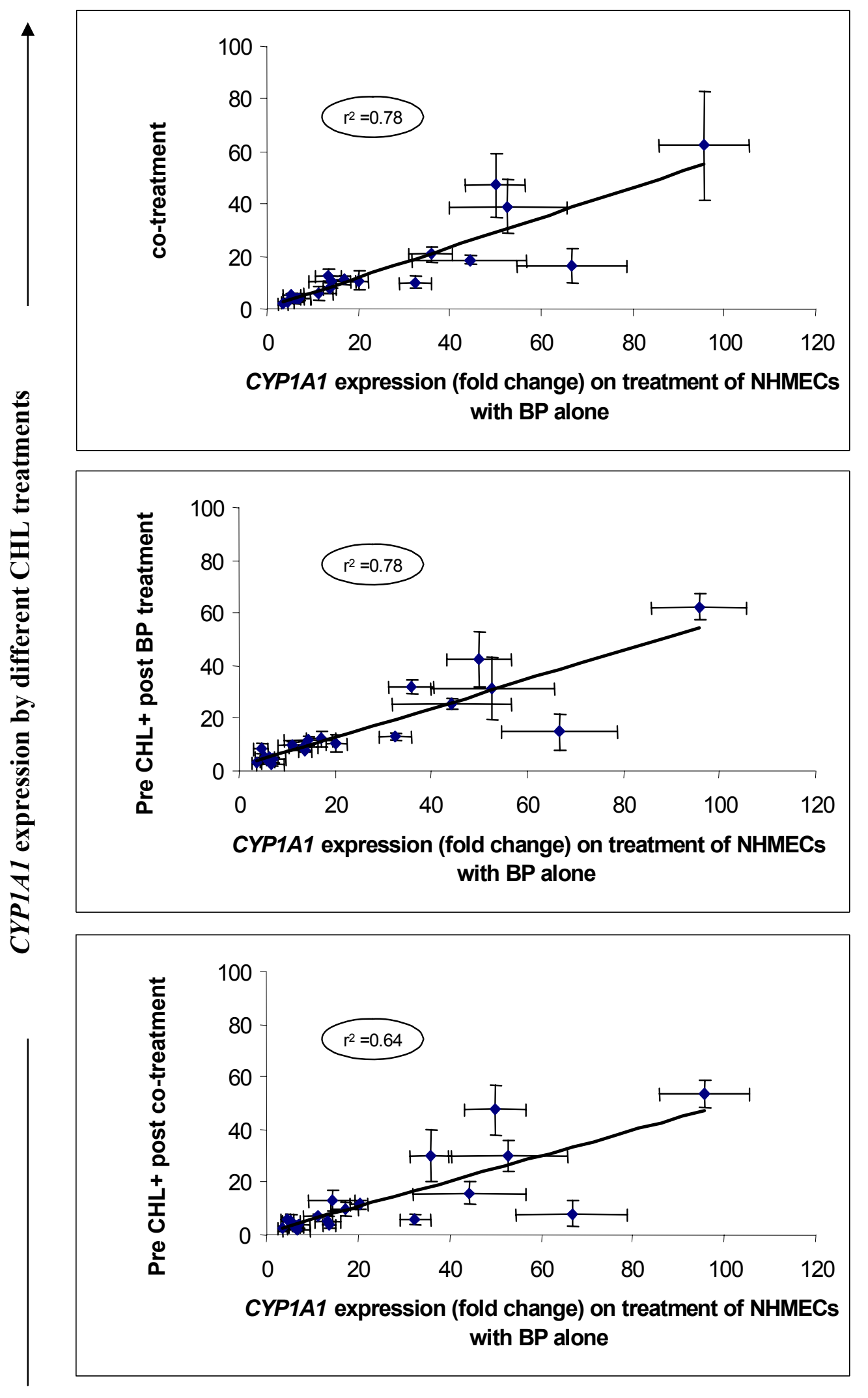

Figure 15: Correlation of CYP1A1 expression in the absence and presence of CHL 


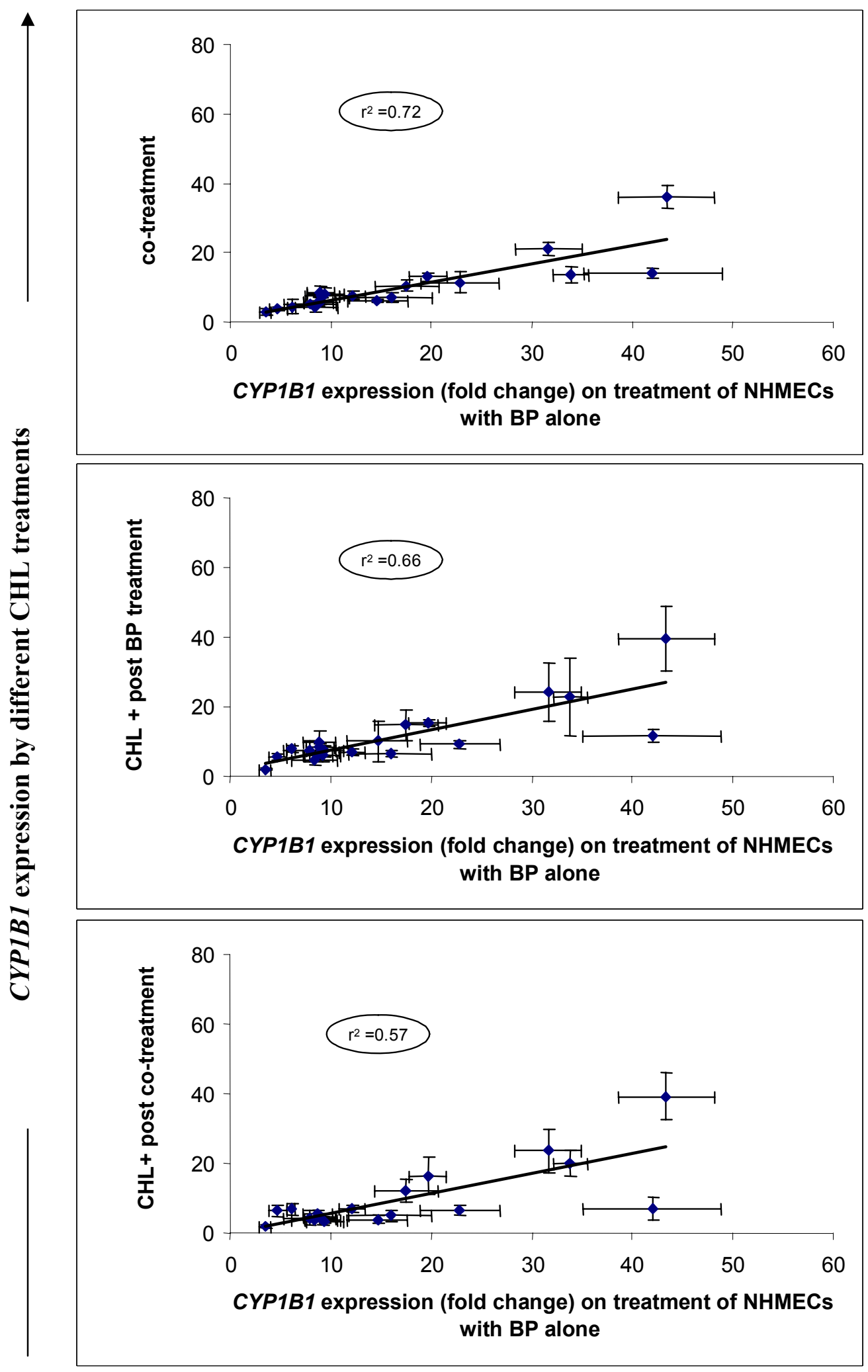

Figure 16: Correlation of CYPIBI expression in the absence and presence of CHL 


\subsection{3 $C Y P 1 A 1$ and $C Y P 1 B 1$ expression in 2 NHMECs on treatment with $\mathrm{BP}$ or $\mathrm{B}[\mathrm{e}] \mathrm{P}$ in the absence or presence of $\mathrm{CHL}$}

With respect to studies on comparison of $\mathrm{BP}$ versus $\mathrm{B}[\mathrm{e}] \mathrm{P}$ in the induction of CYP1A1 and CYP1B1 wide 'inter' cell strain differences were observed in the induction and modulation of both CYP1A1 and CYP1B1 in both M98015 and M98025 on treatment with $\mathrm{BP}$ or $\mathrm{B}[\mathrm{e}] \mathrm{P}$ in the absence or presence of $\mathrm{CHL}$ (Figures 17 and 18).

The induction of both $C Y P 1 A 1$ and $C Y P 1 B 1$ by BP was comparatively higher in M98015 than M98025 (35 fold versus 10 fold for CYP1A1 and 23 fold versus 5 fold for $C Y P 1 B 1)$. Though $\mathrm{B}[\mathrm{e}] \mathrm{P}$ was a very poor inducer of both $C Y P 1 A 1$ and $C Y P 1 B 1$ in $\mathrm{M} 98015$ ( 2 fold and 1 fold, respectively) and M98025 (0.7 fold and 1 fold, respectively) compared to BP, the induction of CYP1A1 by B[e]P was slightly higher in M98015 compared to M98025 (2 fold versus 0.7 fold). The induction $C Y P 1 B 1$ by B[e]P, however, showed little difference between M98015 and M98025.

CHL co-treatment caused a statistically significant decrease of BP induced CYP1A1 expression in M98015 and M98025 (30\% and 37\% respectively, $\mathrm{p}<0.05$ for both). CHL cotreatment also caused a decrease of BP induced CYP1B1 expression in both M98015 and M98025 (42\% and 21\%, respectively) with the reduction in M98015 being statistically significant $(\mathrm{p}<0.05)$. In the case of $\mathrm{B}[\mathrm{e}] \mathrm{P}$, all CHL co-treatments caused an increase in the expression of $\mathrm{B}[\mathrm{e}] \mathrm{P}$ induced $C Y P 1 A 1$ and $C Y P 1 B 1$ in both $\mathrm{M} 98015(148 \%, \mathrm{p}<0.005$ and $219 \%, \mathrm{p}=0.005$, respectively) and M98025 (75\%, $<<0.005$ and $132 \%, \mathrm{p}<0.05$ respectively).

Pre $\mathrm{CHL}+$ post BP treatment did not mitigate BP induced expression of CYP1A1 in M98015 (0.17\% increase) but did mitigated it in M98025 (29\%). The expression of BP induced CYP1B1 was reduced only slightly in M98015 (5\%) but significantly in M98025 
$(50 \%, \mathrm{p}<0.05)$. Pre $\mathrm{CHL}+$ post $\mathrm{BP}$ treatment caused a mitigation of $\mathrm{B}[\mathrm{e}] \mathrm{P}$ induced CYPIAI and CYP1B1 expression in M98015 (3\% and 4\%, respectively) and M98025 (19\% and 54\%, respectively).

Pre CHL + co-treatment did not modulate the expression of BP induced CYPIAl in M98015 but caused a statistically significant decrease in the expression of CYP1A1 in M98025 (40\%, $<<0.05)$. The expression of CYP1B1 was significantly reduced in both M98015 and M98025 (35\% and 47\% respectively, $\mathrm{p}<0.05$ for both). There was a statistically significant increase in the expression of $\mathrm{B}[\mathrm{e}] \mathrm{P}$ induced CYP1A1 in M98015 $(155 \%, \mathrm{p}<0.005)$ and M98025 $(112 \%, \mathrm{p}<0.05)$ upon pre $\mathrm{CHL}+$ co-treatment. The expression of $\mathrm{B}[\mathrm{e}] \mathrm{P}$ induced CYP1B1 was increased in M98015 (62\%, p<0.05) but decreased in M98025 (25\%).

The correlation $\left(\mathrm{r}^{2}\right)$ of BP induced CYPIA1 expression to that of CYPIB1 expression across all treatments for M98015 and M98025 was 0.47 and 0.54 , respectively. The correlation $\left(\mathrm{r}^{2}\right)$ of $\mathrm{B}[\mathrm{e}] \mathrm{P}$ induced $C Y P 1 A 1$ expression to that of $C Y P 1 B 1$ expression across all treatments for M98015 and M98025 was 0.97 and 0.71, respectively. 

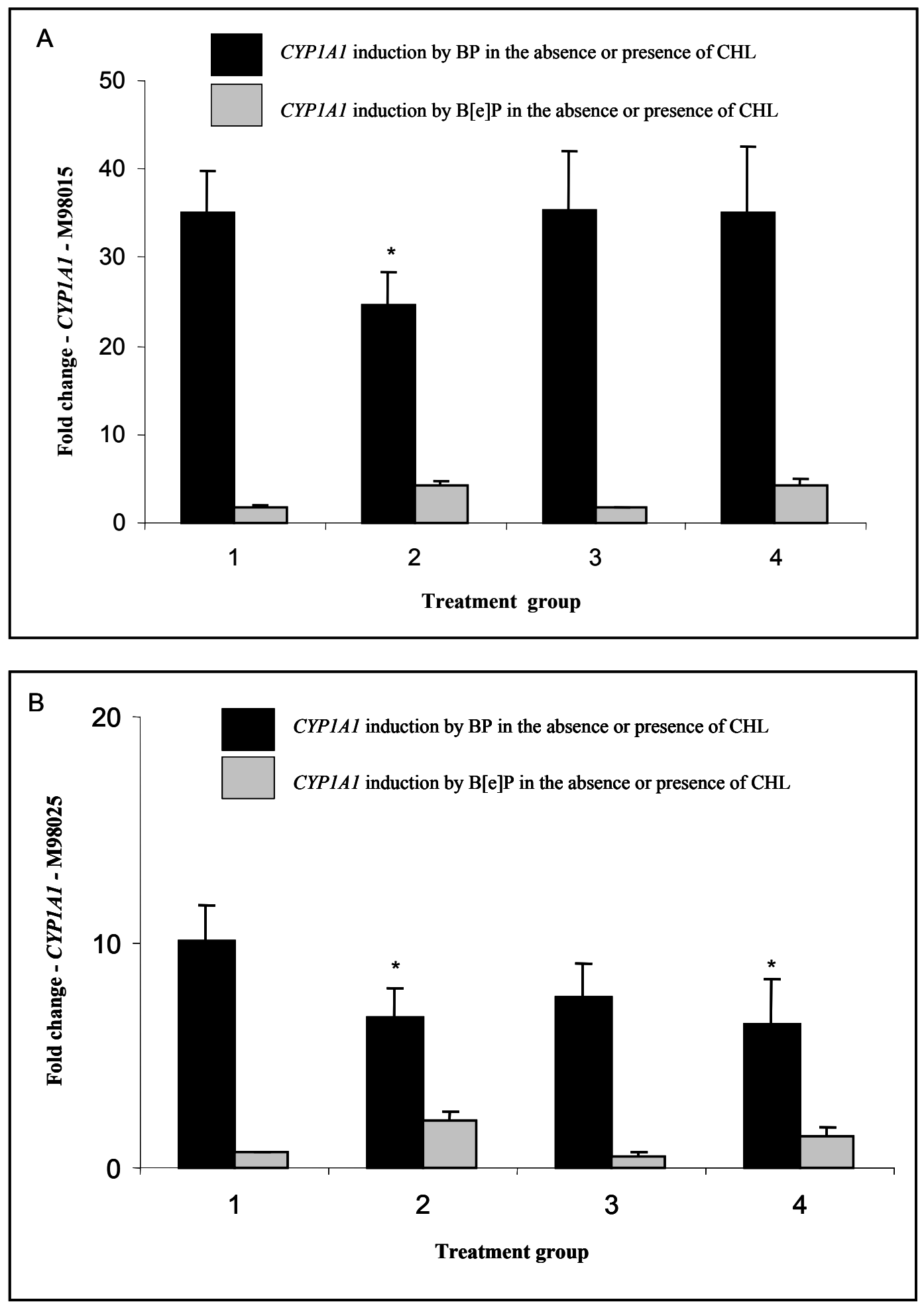

Figure 17: Comparison of CYP1A1 expression by BP or B[e]P in (A) M98015 and (B) M98025 in the absence or presence of CHL.

Treatments: $1=\mathrm{BP}$ alone, $2=$ co-treatment, $3=$ Pre $\mathrm{CHL}+$ post $\mathrm{BP}$ treatment, $4=$ Pre CHL + post co-treatment.

* indicates a statistically significant reduction in CYP1A1 expression compared to BP alone 

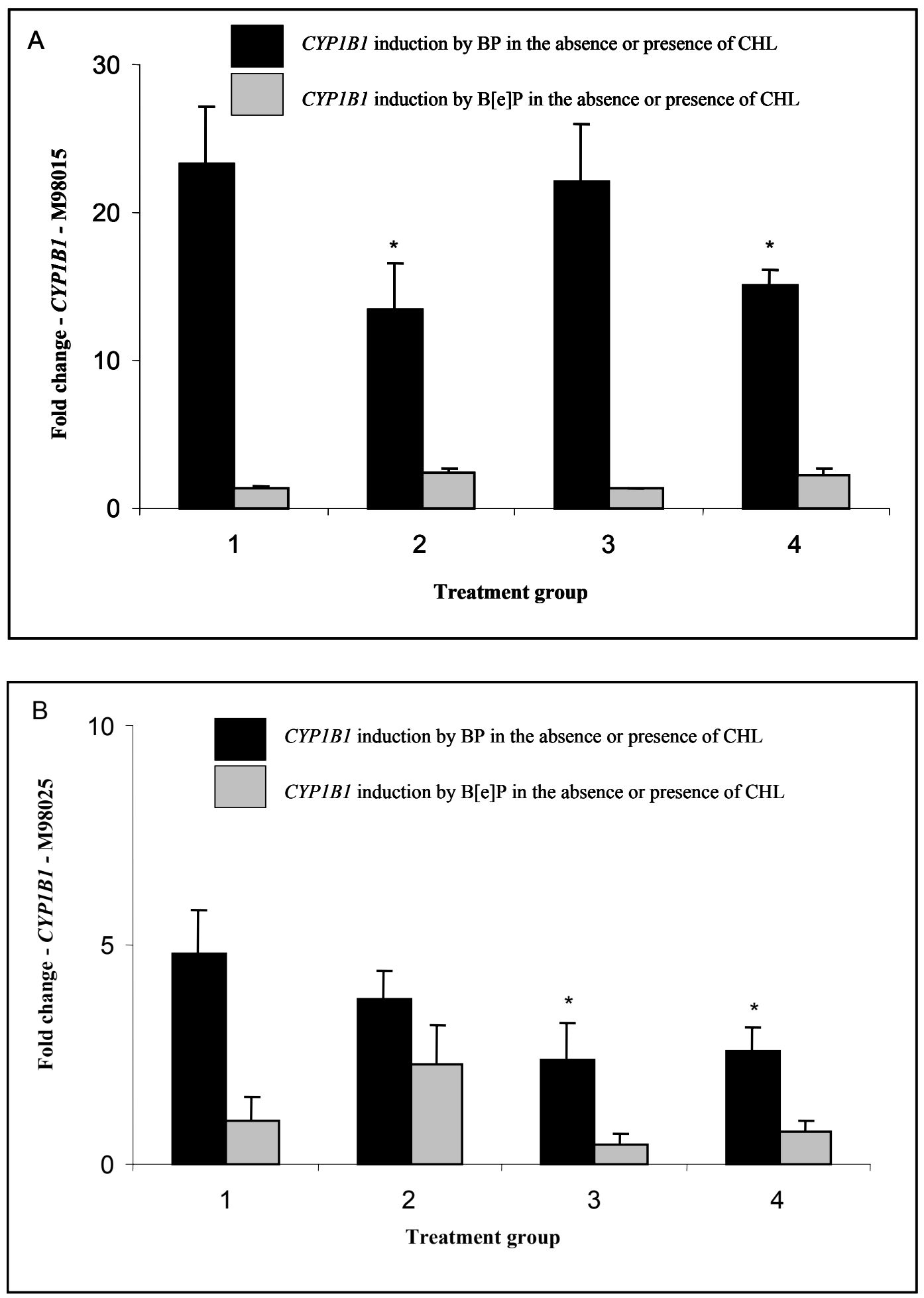

Figure 18: Comparison of CYP1B1 expression by BP or B[e]P in (A) M98015 and (B) M98025 in the absence or presence of CHL.

Treatments: $1=\mathrm{BP}$ alone, $2=$ co-treatment, $3=$ Pre $\mathrm{CHL}+$ post $\mathrm{BP}$ treatment, $4=$ Pre $\mathrm{CHL}$ + post co-treatment.

* indicates a statistically significant reduction in CYP1B1 expression compared to BP alone 


\subsection{4 $C Y P 1 A 1$ and $C Y P 1 B 1$ expression in MCF-7 and M00012 on treatment with BP in the absence or presence of $\mathrm{CHL}$}

Comparison of MCF-7 with a NHMEC (M00012) across various CHL treatments (as detailed in Table 3 of Materials and Methods) revealed that though basal CYP1A1 expression was comparable for both MCF-7 and M00012 $(\triangle \mathrm{Ct}=12.8$ for both $), C Y P 1 B 1$ expression was much higher in MCF-7 $(\Delta \mathrm{Ct}=4.37)$ as compared to M00012 $(\Delta \mathrm{Ct}=7.92)$. The basal expression level of $C Y P 1 B 1$ in MCF-7 cells was 348 times that of CYP1A1 whereas that of M00012 was only 29 times that of CYP1A1. The basal expression of CYP1B1 in MCF-7 was nearly 12 times that in M00012. The induction of CYP1A1 by BP alone was 114 fold whereas that of $C Y P 1 B 1$ was only 5 fold in MCF-7. All CHL treatments caused an increase of BP induced CYPIA1 $(16 \%, 22 \%$ and $23 \%$ increase on co-treatment, pre $\mathrm{CHL}+$ post $\mathrm{BP}$ treatment and pre $\mathrm{CHL}+$ post co-treatment, respectively) and $C Y P 1 B 1(11 \%, 12 \%$ and 33\% increase on co-treatment, pre CHL + post BP treatment and pre CHL + post co-treatment respectively) expression. In the case of M00012, all CHL treatments caused a mitigation of BP induced CYPIA1 $(36 \%, 29 \%$ and $43 \%$ decrease on co-treatment, pre $\mathrm{CHL}+$ post $\mathrm{BP}$ treatment and pre $\mathrm{CHL}+$ post co-treatment, respectively) and $C Y P 1 B 1(41 \%, 16 \%$ and $32 \%$ decrease on co-treatment, pre $\mathrm{CHL}+$ post $\mathrm{BP}$ treatment and pre $\mathrm{CHL}+$ post co-treatment, respectively).

The overall correlation of $C Y P 1 A 1$ expression to that of $C Y P 1 B 1$ expression across all treatments was moderate for MCF-7 and good for M00012 $\left(\mathrm{r}^{2}=0.61\right.$ and 0.80 for MCF-7 and M00012, respectively).

A comparison of the extent of CYP1 expression between MCF-7 and 20 NHMECs used in the above studies was also carried out in order to have a comparison against response 
patterns of a broad panel of cell strains. Such a comparison against a broad panel would take into account inter-cell strains differences in induction and modulation. The basal CYP1A1 and CYPIB1 expression in MCF-7 was higher than the average basal CYP1A1 and CYPIB1 expression across all the $20 \mathrm{NHMECs}(\triangle \mathrm{Ct}=12.81$ and 4.37 for $C Y P 1 A 1$ and $C Y P 1 B 1$ in MCF-7 versus 13.46 and 7.71 for CYP1A1 and CYPIB1 in the 20 NHMECs). This corresponds to a 2 fold higher expression of CYP1A1 and a10 fold higher expression level of CYP1B1 in MCF-7 versus the 20 NHMECs. The average basal CYP1B1 expression in the 20 NHMECs was 53 fold that of CYP1A1. The expression patterns of CYP1A1 and CYP1B1 on treatment of the 20 NHMECs with BP alone or in the presence of different CHL treatments are presented in Tables 11 and 12, respectively. The induction of CYP1A1 by BP alone in MCF-7 (114 fold) was much higher than even the highest inducer in the 20 NHMECs (96 fold in M99016). This held true for even the other CHL treatments (co-treatment, pre CHL + post $\mathrm{BP}$ treatment and pre $\mathrm{CHL}+$ post co-treatment). With regard to CYPIBI expression, the expression in MCF-7 by BP in the absence and presence of CHL was in the range of the 20 NHMECs. Figures 19 and 20 depict the expression patterns of CYP1A1 and CYP1B1, respectively in MCF-7 and M00012 across the different treatment groups. In each case the inset shows the expression pattern of MCF-7 in comparison with the other 20 NHMECs. 

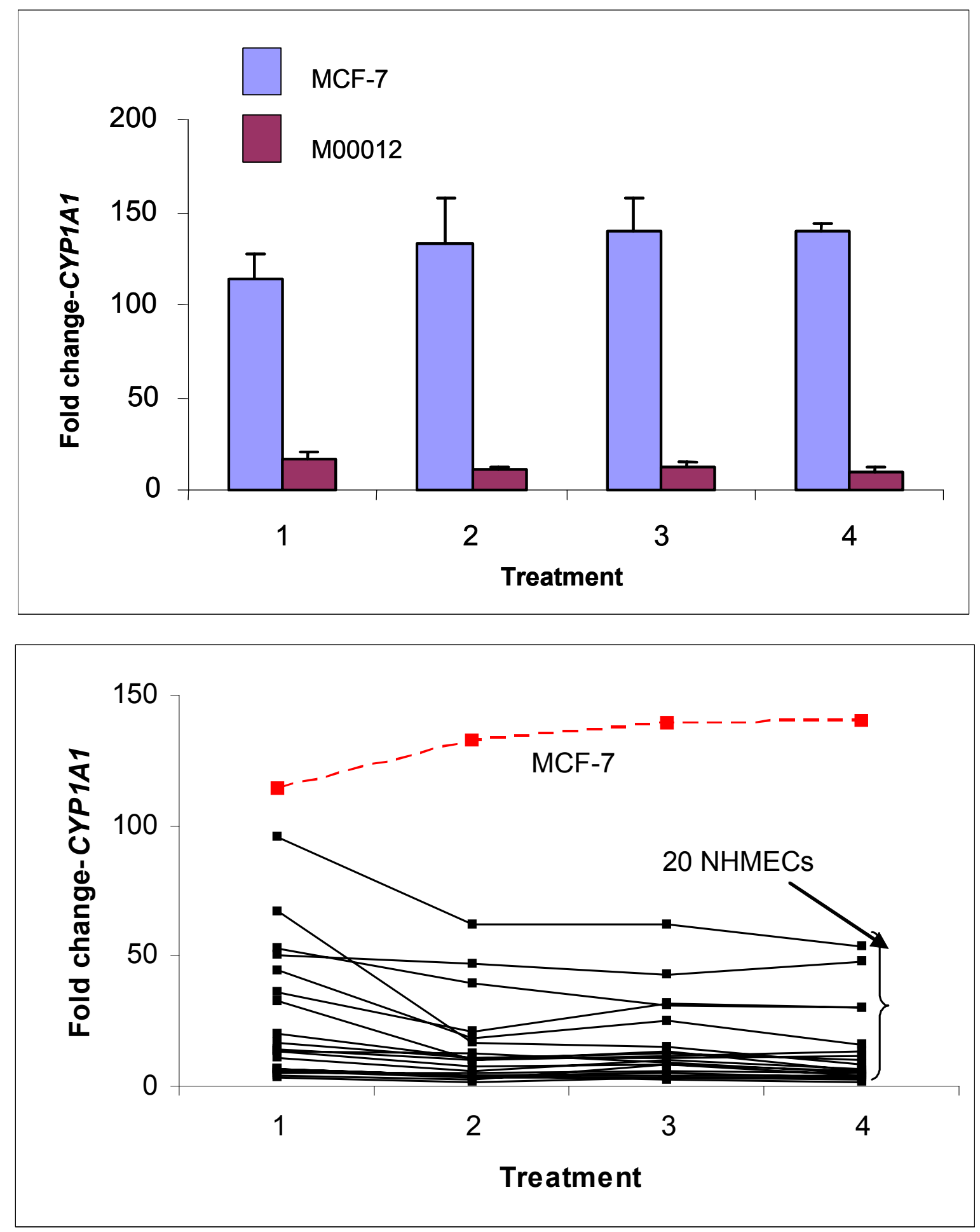

Figure 19: (A) CYP1A1 expression patterns on treatment of MCF-7 cells or M00012 (a NHMEC) with BP in the absence or presence of CHL. (B) CYP1A1 expression patterns in MCF-7 versus 20 NHMECs. The expression of CYP1A1 in MCF-7 cells is much higher and different compared to the NHMECs.

Treatments: $1=\mathrm{BP}$ alone, $2=$ co-treatment, $3=$ Pre $\mathrm{CHL}+$ post $\mathrm{BP}$ treatment, $4=$ Pre $\mathrm{CHL}$ + post co-treatment. 

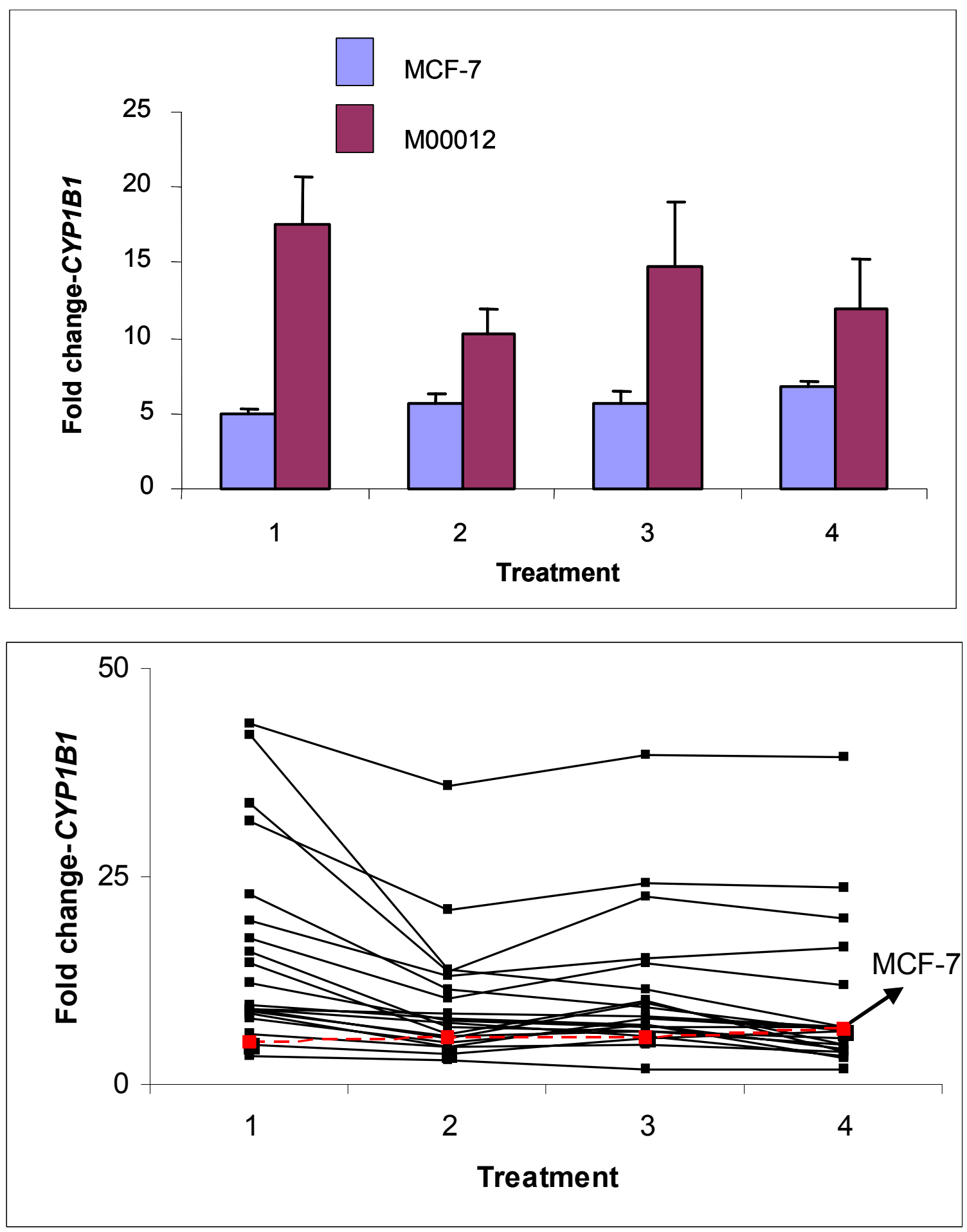

Figure 20: (A) CYP1B1 expression patterns on treatment of MCF-7 cells or M00012 (a NHMEC) with BP in the absence or presence of CHL. (B) CYP1B1 expression patterns in MCF-7 versus 20 NHMECs. The expression of CYP1B1 in MCF-7 cells was in the range of the NHMECs.

Treatments: $1=\mathrm{BP}$ alone, $2=$ co-treatment, $3=$ Pre $\mathrm{CHL}+$ post $\mathrm{BP}$ treatment, $4=$ Pre CHL + post co-treatment. 


\subsubsection{CYP1A1 and $C Y P 1 B 1$ expression in MCF-7 and M00012 on treatment with BP in the absence or presence of different CHL concentrations}

With regard to the effect of a range of $\mathrm{CHL}$ concentrations on $\mathrm{BP}$ induced CYP1A1 and $C Y P 1 B 1$ expression in MCF-7 cells, BP induced $C Y P 1 A 1$ expression 38 fold while that of CYP1B1 was induced only 3 fold. Pre CHL + post co-treatment enhanced BP induced CYP1A1 expression of cells at low concentrations of CHL $(2 \mu \mathrm{M}, 3 \mu \mathrm{M}$ and $4 \mu \mathrm{M})$ in MCF-7 cells. The induction of $C Y P 1 A 1$ was 100 fold, 260 fold and 48 fold on pre-treatment with CHL $2 \mu \mathrm{M}, 3 \mu \mathrm{M}$ or $4 \mu \mathrm{M}$, respectively followed by co-treatment. However, higher concentrations of CHL ( $8 \mu \mathrm{M}$ and $16 \mu \mathrm{M})$ mitigated BP induced CYP1A1 expression on pretreatment with CHL followed by the respective post co-treatment, though there did not appear to be a concentration dependent response. The fold induction of $C Y P 1 A 1$ on pre $8 \mu \mathrm{M}$ $\mathrm{CHL}+$ post co-treatment and pre $16 \mu \mathrm{M} \mathrm{CHL}+$ post co-treatment was 24 fold and 36 fold, respectively. The expression of $C Y P 1 B 1$ however was not altered across the different $\mathrm{CHL}$ concentrations in MCF-7. All CHL concentrations caused a slight increase of BP induced $C Y P 1 B 1$ expression. $C Y P 1 B 1$ expression on treatment of MCF-7 cells with BP alone and in the presence of $2 \mu \mathrm{M}, 3 \mu \mathrm{M}, 4 \mu \mathrm{M}, 8 \mu \mathrm{M}$ and $16 \mu \mathrm{M}$ CHL in a pre CHL + post co-treatment were $3,3,4,4,4$ and 3 fold, respectively.

In the case of M00012, highest CYP1A1 expression was in the group treated with BP alone (23 fold) while all CHL treatments except pre $8 \mu \mathrm{M}+$ post co-treatment mitigated BP induced CYPIA1 expression to different extents $(21,13,19,23$ and 2 fold on pre-treatment with $2 \mu \mathrm{M}, 3 \mu \mathrm{M}, 4 \mu \mathrm{M}, 8 \mu \mathrm{M}$ and $16 \mu \mathrm{M}$ CHL followed by co-treatment, respectively). With respect to $C Y P 1 B 1$ expression, again there did not appear to be a concentration dependent response. The induction of $C Y P 1 B 1$ by BP was 8 fold whereas the induction in the 
presence of $2 \mu \mathrm{M}, 3 \mu \mathrm{M}, 4 \mu \mathrm{M}, 8 \mu \mathrm{M}$ and $16 \mu \mathrm{M}$ CHL pre-treatment followed by cotreatment was 10, 5, 3, 16 and 3 fold, respectively. The correlation $\left(\mathrm{r}^{2}\right)$ of CYP1A1 expression to CYPIBI expression by $\mathrm{BP}$ in the absence or presence of a range of $\mathrm{CHL}$ concentrations in MCF-7 cells was 0.24 while that in M00012 was 0.41 . The expression patterns of CYP1A1 or CYP1B1 in MCF-7 and M00012 by BP in the absence or presence of a range of CHL concentrations are shown in Figures 21 and 22, respectively. 


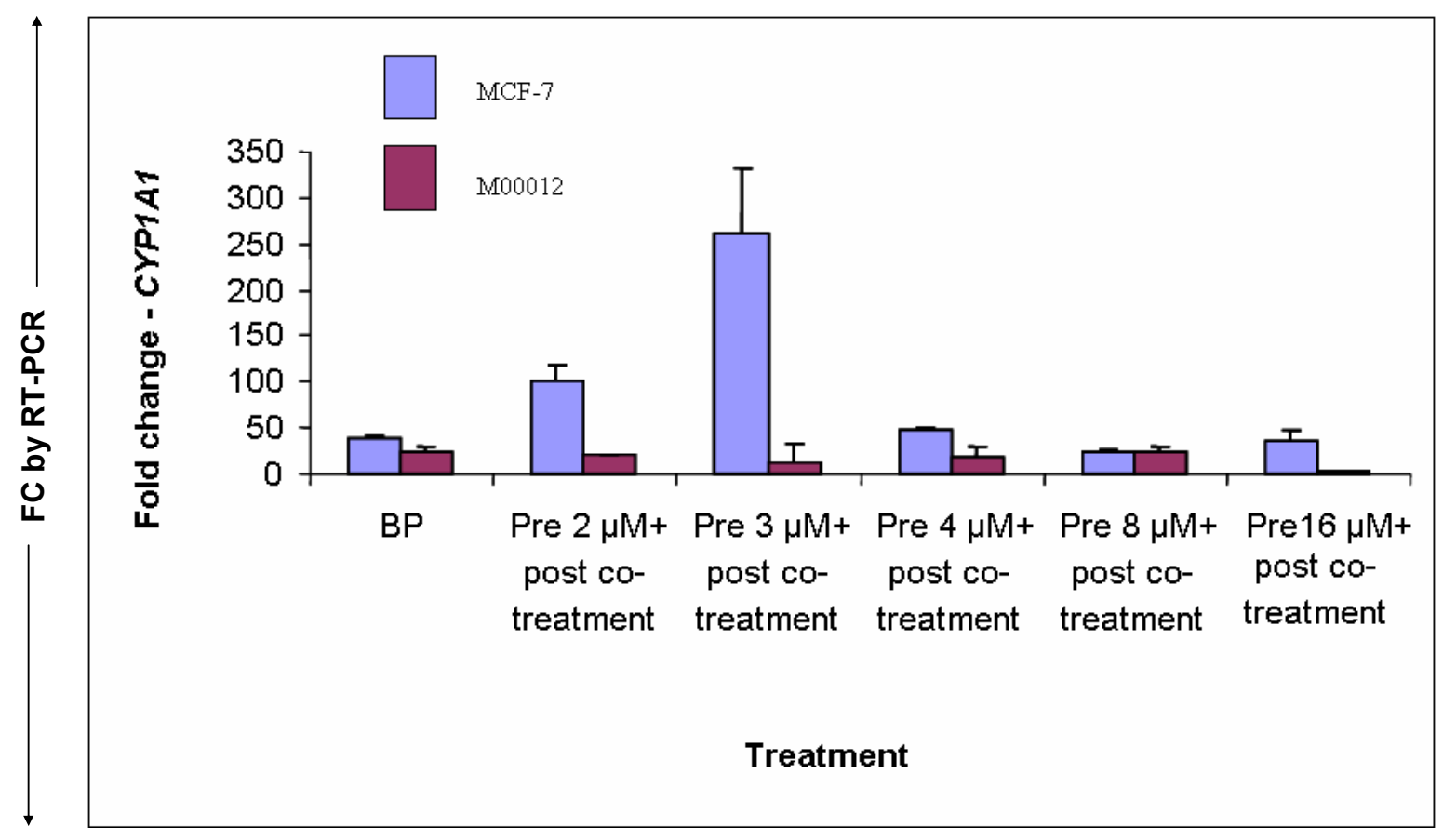

Figure 21: CYP1A1 expression patterns in MCF-7 and M00012 by BP in the absence or presence of a range of CHL concentrations.

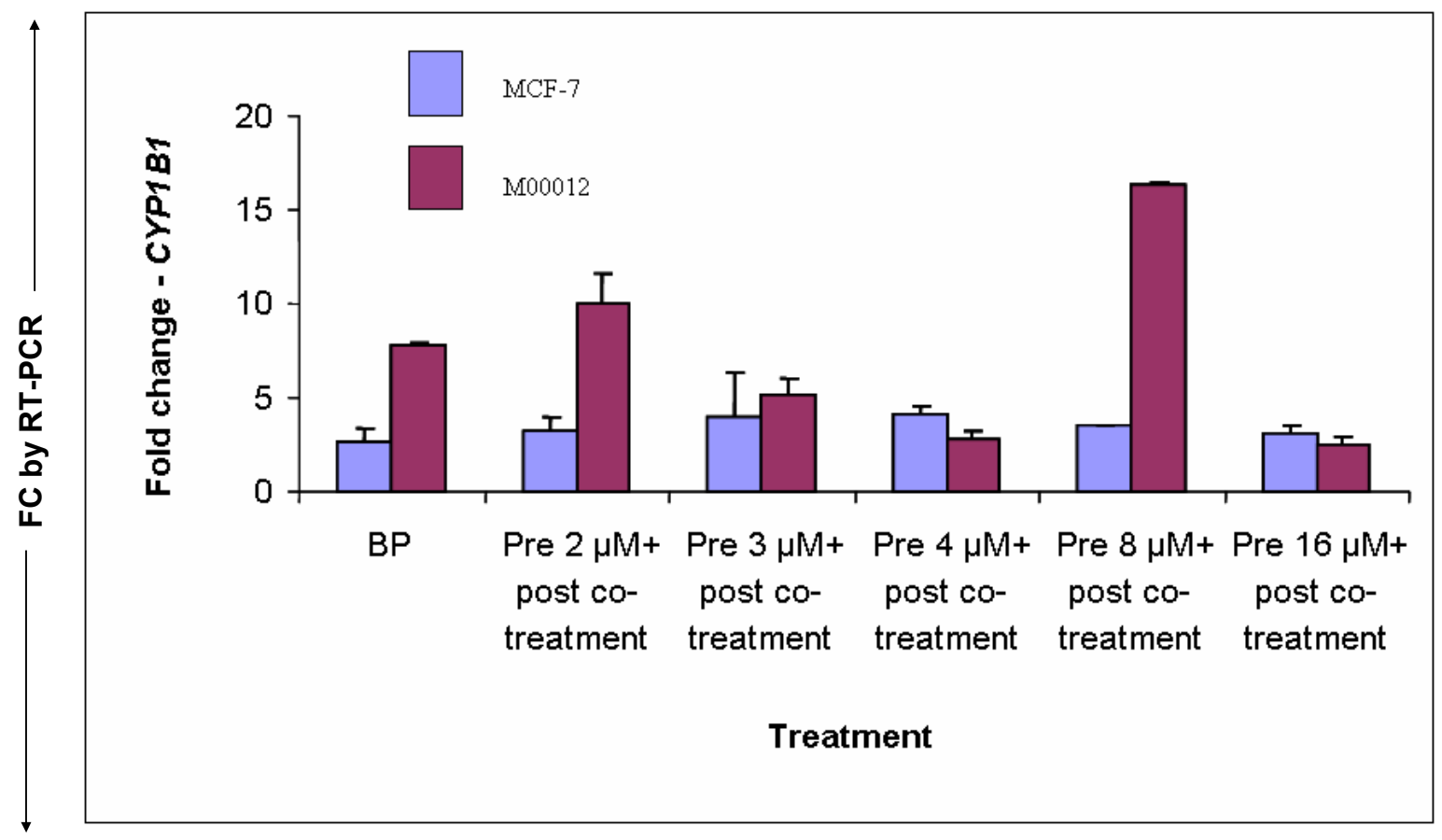

Figure 22: $C Y P 1 B 1$ expression patterns in MCF-7 and M00012 by BP in the absence or presence of a range of CHL concentrations. 


\subsection{DNA adducts}

\subsubsection{BP-DNA adducts in 20 NHMECs on treatment with BP in the absence or presence of CHL}

The levels of BP-DNA adducts across the 20 NHMECs exhibited considerable interindividual variation with highest levels being in the BP treated group (Table 14). All CHL treatments lowered BP-DNA adducts in most cell strains except in M99021 (which exhibited an increase in adduct levels upon co-treatment and pre CHL + post BP treatment) and M99025 (which showed no reduction in adduct levels upon CHL co-treatment). The number of BP-DNA adducts in the BP treated group ranged from 2.5 adducts $/ 10^{8}$ nucleotides (in M99005) to 57.5 adducts $/ 10^{8}$ nucleotides (M99015).

CHL co-treatment caused a decrease in BP-DNA adduct levels in 18/20 cell strains (13$55 \%$ reduction) while increasing it in M99021 (5\% increase) and having no effect in M99025. The average reduction in BP-DNA adducts by CHL co-treatment was 33\%. Pre CHL + post BP treatment caused a reduction in BP- DNA adduct levels in 19/20 cell strains (3-67\%) while increasing it in M99021 (12\% increase). The average reduction in BP-DNA adducts by CHL pretreatment followed by post BP treatment was $28 \%$. Pre CHL + post co-treatment caused a reduction in BP-DNA adducts in all cell strains (28-87\%). The average reduction in BP-DNA adducts by CHL pre-treatment followed by post co- treatment was 53\%. As evident, the maximum reduction in BP-DNA adducts for most cell strains was by pre $\mathrm{CHL}+$ post co-treatment except in M99015, M98019 and M99005.

There was a good correlation between the levels of BP-DNA adducts in the presence of BP alone and by $\mathrm{CHL}$ co-treatment $\left(\mathrm{r}^{2}=0.90\right)$ as well as pre $\mathrm{CHL}+$ post BP treatment $\left(\mathrm{r}^{2}=0.89\right)$. However, their correlation in the presence of pre CHL + post co-was only moderate $\left(r^{2}=0.68\right)$. 
Table14: Inter-individual variations in BP-DNA adduct formation on exposure of NHMECs to either BP alone or in combination with $\mathrm{CHL}$

\begin{tabular}{|c|c|c|c|c|}
\hline \multicolumn{5}{|c|}{ BP-DNA adducts $/ 10^{8}$ nt. } \\
\hline \multicolumn{5}{|c|}{ Treatment } \\
\hline Cell strain & 1 & 2 & 3 & 4 \\
\hline M99005 & $2.45 \pm 1.34$ & $1.25 \pm 1.20$ & $2.20 \pm 2.26$ & $1.50 \pm 1.70$ \\
\hline M99025 & $3.15 \pm 0.64$ & $3.15 \pm 0.21$ & $2.50 \pm 1.27$ & $1.35 \pm 0.07$ \\
\hline M98014 & $4.67 \pm 0.61$ & $2.24 * \pm 0.35$ & $2.87 * \pm 0.15$ & $2.10^{*} \pm 0.73$ \\
\hline M99004 & $4.75 \pm 0.49$ & $2.75 \pm 0.64$ & $2.30 \pm 0.99$ & $1.90 \pm 0.85$ \\
\hline M98019 & $5.09 \pm 0.94$ & $3.05^{*} \pm 1.29$ & $3.22 * \pm 0.81$ & $3.59 \pm 1.11$ \\
\hline M98035 & $5.85 \pm 3.89$ & $2.70 \pm 1.13$ & $5.70 \pm 1.56$ & $2.65 \pm 1.34$ \\
\hline M99003 & $7.25 \pm 1.48$ & $3.30 \pm 0.28$ & $3.45 \pm 1.20$ & $1.30 \pm 0.42$ \\
\hline M98015 & $8.30 \pm 4.95$ & $5.50 \pm 3.25$ & $5.45 \pm 4.03$ & $4.30 \pm 4.10$ \\
\hline M98030 & $9.47 \pm 1.52$ & $8.12 \pm 3.26$ & $5.53 \pm 1.78$ & $4.00 \pm 1.63$ \\
\hline M99021 & $13.66 \pm 3.16$ & $14.29 \pm 1.49$ & $15.32 \pm 3.76$ & $10.21 \pm 5.40$ \\
\hline M98025 & $15.10 \pm 2.40$ & $5.10 \pm 0.57$ & $4.95 \pm 1.77$ & $3.70 * \pm 2.40$ \\
\hline M99006 & $17.32 \pm 3.70$ & $13.59 \pm 3.25$ & $15.49 \pm 5.75$ & $12.56 \pm 4.14$ \\
\hline M98011 & $20.83 \pm 13.03$ & $15.33 \pm 13.06$ & $17.84 \pm 9.19$ & $11.73 \pm 9.25$ \\
\hline M00012 & $21.0 \pm 4.53$ & $14.65 \pm 1.77$ & $16.35 \pm 3.46$ & $10.85 \pm 3.32$ \\
\hline M98013 & $23.13 \pm 1.96$ & $14.45^{*} \pm 2.75$ & $12.18 * \pm 1.23$ & $3.00 * \pm 0.91$ \\
\hline M00004 & $33.41 \pm 6.12$ & $27.05 \pm 13.72$ & $28.33 \pm 9.63$ & $19.83 \pm 12.34$ \\
\hline M99016 & $34.00 \pm 3.39$ & $23.85 \pm 3.61$ & $25.45 \pm 2.62$ & $12.50 * \pm 1.41$ \\
\hline M98016 & $53.38 \pm 2.52$ & $26.05^{*} \pm 2.32$ & $25.59 * \pm 7.00$ & $7.04 * \pm 1.37$ \\
\hline M98026 & $54.46 \pm 29.74$ & $47.29 \pm 26.00$ & $42.49 \pm 21.40$ & $32.49 \pm 18.19$ \\
\hline M99015 & $57.49 \pm 16.23$ & $34.99 \pm 15.88$ & $51.40 \pm 12.08$ & $35.43 \pm 10.79$ \\
\hline
\end{tabular}

Treatments - 1-BP alone, 2-co-treatment, 3-Pre CHL+ post BP treatment, 4-Pre CHL + co-treatment (Details of treatments in Materials and methods)

Numbers shown represent fold change \pm standard deviation $(n=2)$ as measured by CIA

$*$ = statistically significant reduction in expression $(\mathrm{p} \leq 0.05)$ compared to expression induced by BP alone 


\subsubsection{BP-DNA and B[e]P-DNA adducts in 2 NHMECs on treatment with $\mathrm{BP}$ or $\mathrm{B}[\mathrm{e}] \mathrm{P}$ in the absence or presence of $\mathrm{CHL}$}

In the comparison between M98015 and M98025 on treatment with BP or B[e]P in the absence or presence of CHL, both cell strains had the highest number of BP-DNA adducts in cells treated with BP alone (8.3 and 15.1 adducts $/ 10^{8}$ nucleotides in M98015 and M98025, respectively). All CHL treatments reduced the levels of BP-DNA adducts in M98015 (34\%, 34\% and 48\% reduction for co-treatment, pre CHL + post BP and pre CHL + post co-treatment, respectively) and M98025 (66\%, 67\% and 76\% for co-treatment, pre CHL + post $\mathrm{BP}$ and pre $\mathrm{CHL}+$ post co-treatment, respectively). $\mathrm{B}[\mathrm{e}] \mathrm{P}-\mathrm{DNA}$ adducts were not detectable by synchronous fluorescence spectrometry in $\mathrm{B}[\mathrm{e}] \mathrm{P}$ as well as the CHL treatment groups $(\mathrm{B}[\mathrm{e}] \mathrm{P}$ co-treatment, pre $\mathrm{CHL}+$ post $\mathrm{B}[\mathrm{e}] \mathrm{P}$ treatment and pre $\mathrm{CHL}+$ post $\mathrm{B}[\mathrm{e}] \mathrm{P}$ cotreatment ) in both M98015 and M98025. Benzo(a)pyrene-r-7,t-8,c-9,t-10-tetrahydrotetrol was used as a standard to construct the standard curve and the synchronous spectra of one of the dilutions is shown in Figure 23. Similarly, the synchronous spectra of the B[e]P treated cells in M98015 and M98025 are shown in Figure 24. 


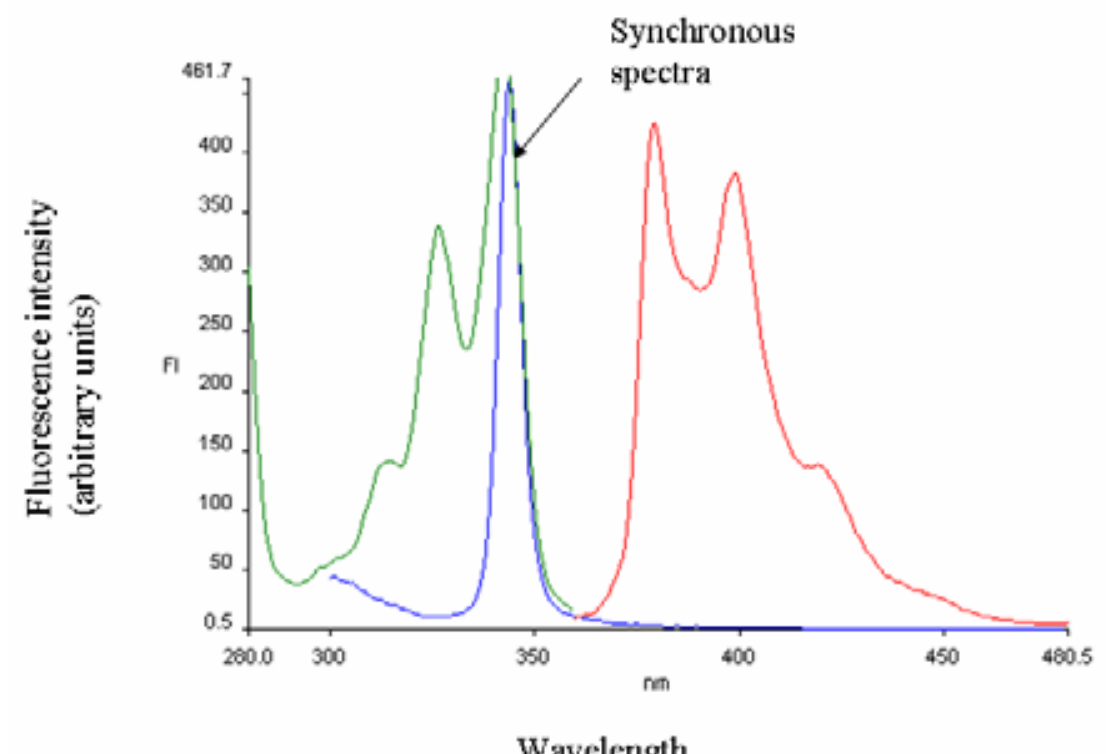

Wavelength

Figure 23: Synchronous fluorescence spectra obtained from standard benzo(a)pyrene-r-7,t-8,c-9,t-10-tetrahydrotetrol. The spectra shown here was generated using $80 \mathrm{fM}$ of the standard
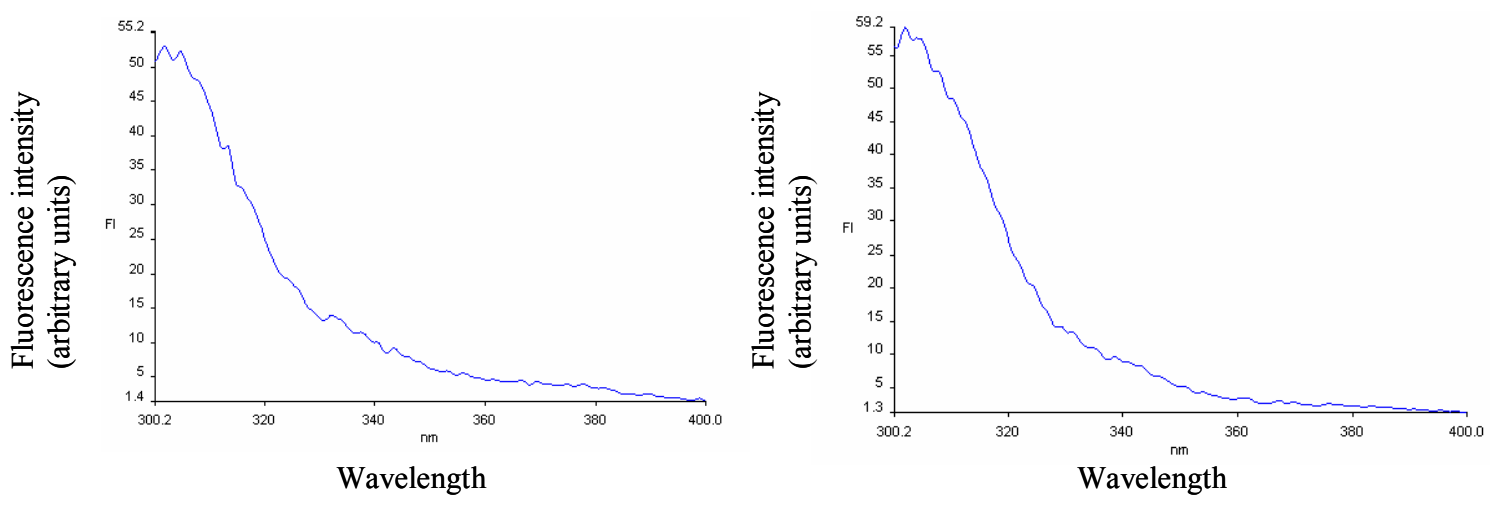

Figure 24: Synchronous spectra of the B[e]P treated cells (A) M98015 and (B) M98025 


\subsubsection{BP-DNA adducts in MCF-7 and M00012 or 20 NHMECs on treatment with BP in the absence or presence of $\mathrm{CHL}$}

Comparison of adducts formed in MCF-7 versus M00012 (NHMEC) following treatment with $\mathrm{BP}$ alone or in the presence of $\mathrm{CHL}, \mathrm{MCF}-7$ exhibited adduct levels greater than 25 orders of magnitude of that of M00012 for all the treatment groups (BP alone, cotreatment, pre $\mathrm{CHL}+$ post $\mathrm{BP}$ treatment and pre $\mathrm{CHL}+$ post co-treatment). $609 \mathrm{BP}-\mathrm{DNA}$ adducts $/ 10^{8}$ nucleotides were detected in MCF-7 on treatment with BP alone as opposed to only 21 BP-DNA adducts $/ 10^{8}$ nucleotides in M00012. None of the CHL treatments mitigated BP-DNA adduct levels in MCF-7 (3\% reduction on co-treatment, 17 and 6\% increase, pre $\mathrm{CHL}+$ post $\mathrm{BP}$ treatment and pre $\mathrm{CHL}+$ post co-treatment, respectively). M00012 on the other hand exhibited a reduction of BP-DNA adduct levels in all CHL treatment groups $(30 \%, 22 \%$ and $48 \%$ reduction on co-treatment, pre $\mathrm{CHL}+$ post $\mathrm{BP}$ treatment and pre CHL + post co-treatment, respectively).

In comparison with 20 NHMECs, MCF-7 cells exhibited adduct levels greater than 10 orders of magnitude than the highest number of adducts formed in the 20 NHMECs. This was true across all treatment groups $(\mathrm{BP}$, co-treatment, pre $\mathrm{CHL}+$ post $\mathrm{BP}$ treatment and pre $\mathrm{CHL}+$ post co-treatment). The average reduction of BP-DNA adducts across different $\mathrm{CHL}$ treatments among the 20 NHMECs were $33 \%, 28 \%$ and $53 \%$ on co-treatment, pre CHL + post $\mathrm{BP}$ treatment and pre $\mathrm{CHL}+$ post co-treatment, respectively as opposed to no mitigation in BP-DNA adduct levels in MCF-7 across the different CHL treatments. The adduct levels in MCF-7 and M00012 on treatment with BP in the absence or presence of CHL are presented in Figure 25. 


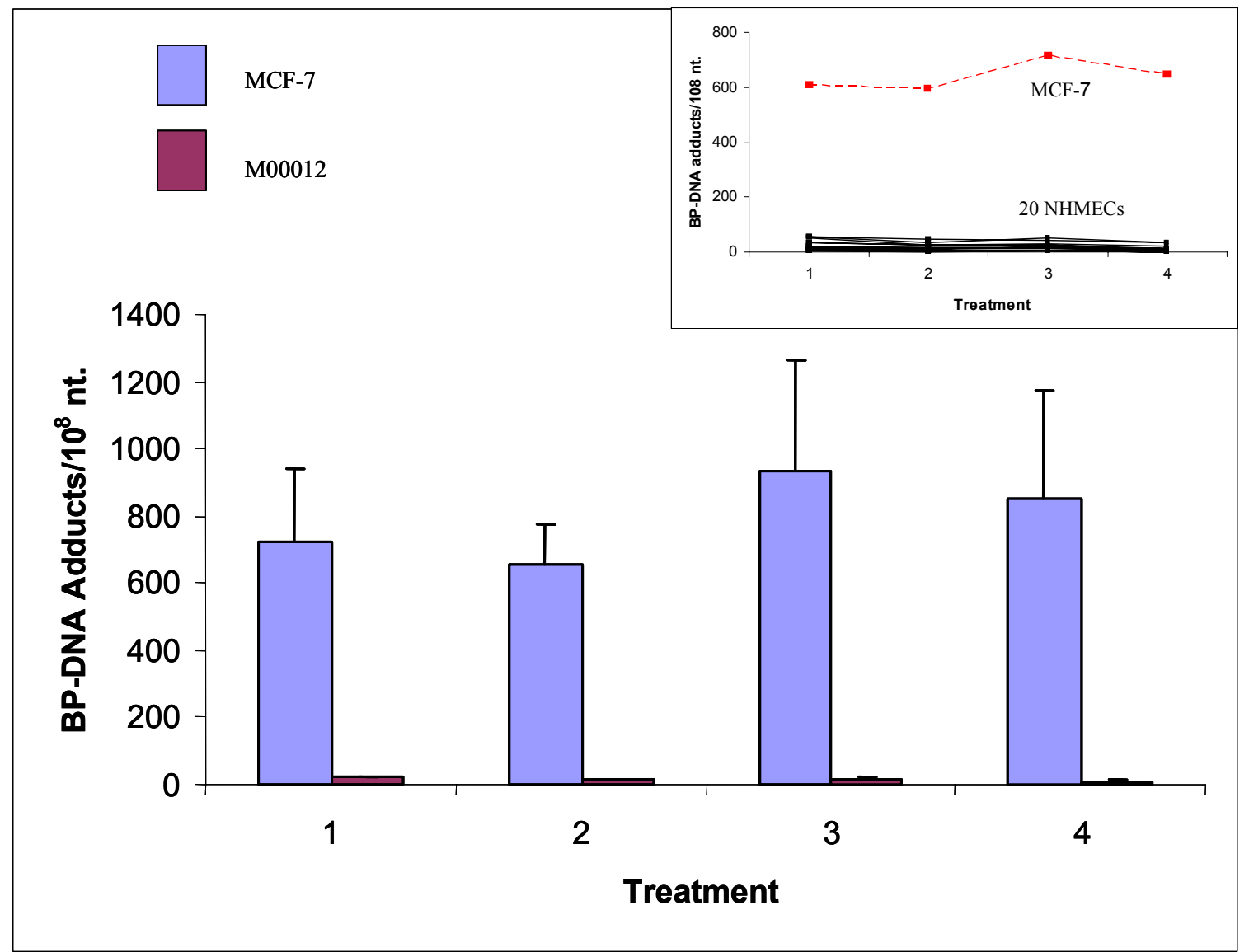

Figure 25: BP-DNA adduct levels $/ 10^{8}$ nucleotides in MCF-7 and M00012 on treatment with BP in the absence or presence of different CHL treatments. The inset on the right shows the BP-DNA adduct levels in MCF-7 versus that in 20 NHMECs. Treatments: $1=\mathrm{BP}$ alone, $2=$ co-treatment, $3=$ Pre $\mathrm{CHL}+$ post $\mathrm{BP}$ treatment, $4=$ Pre $\mathrm{CHL}+$ post co-treatment. 


\subsubsection{BP-DNA adducts in MCF-7 and M00012 on treatment with BP in the absence or presence of different CHL concentrations}

When compared across a range of CHL concentrations, CHL did not imitate the level of BP-DNA adducts across different CHL concentrations in MCF-7 cells. There was no concentration dependent response and even the highest concentration of CHL $(16 \mu \mathrm{M}) \mathrm{did}$ not cause any reduction in the level of BP-DNA adducts. However, there was concentration dependent reduction in the levels of BP-DNA adducts in M00012 for all CHL concentrations except for pre-treatment with $2 \mu \mathrm{M} \mathrm{CHL}$ followed by co- treatment ( $8 \%$ increase compared to cells treated with BP alone). The reduction in BP-DNA adduct levels across all the other CHL treatments were $19 \%, 25 \%, 36 \%$ and $66 \%$ for pre-treatment with $3 \mu \mathrm{M}, 4 \mu \mathrm{M}, 8 \mu \mathrm{M}$ and $16 \mu \mathrm{M}$ CHL followed by co-treatment respectively. The reduction in adduct levels at the higher concentrations of CHL $(8 \mu \mathrm{M}$ and $16 \mu \mathrm{M})$ was significant $(\mathrm{p}<0.05)$. The levels of BPDNA adducts in MCF-7 were more than 13 fold that in M00012 for BP at the lower concentrations of CHL (BP, $2 \mu \mathrm{M}$ and $3 \mu \mathrm{M}$ CHL pre-treatment followed by co-treatment), more than 24 fold for both $4 \mu \mathrm{M}$ and $8 \mu \mathrm{M}$ CHL pre-treatment followed by co-treatment) and 82 fold for the highest concentration of CHL (pre-treatment with CHL $16 \mu \mathrm{M}$ followed by co-treatment). The response patterns of MCF-7 and M00012 across a range of CHL concentrations are shown in Figure 26. 


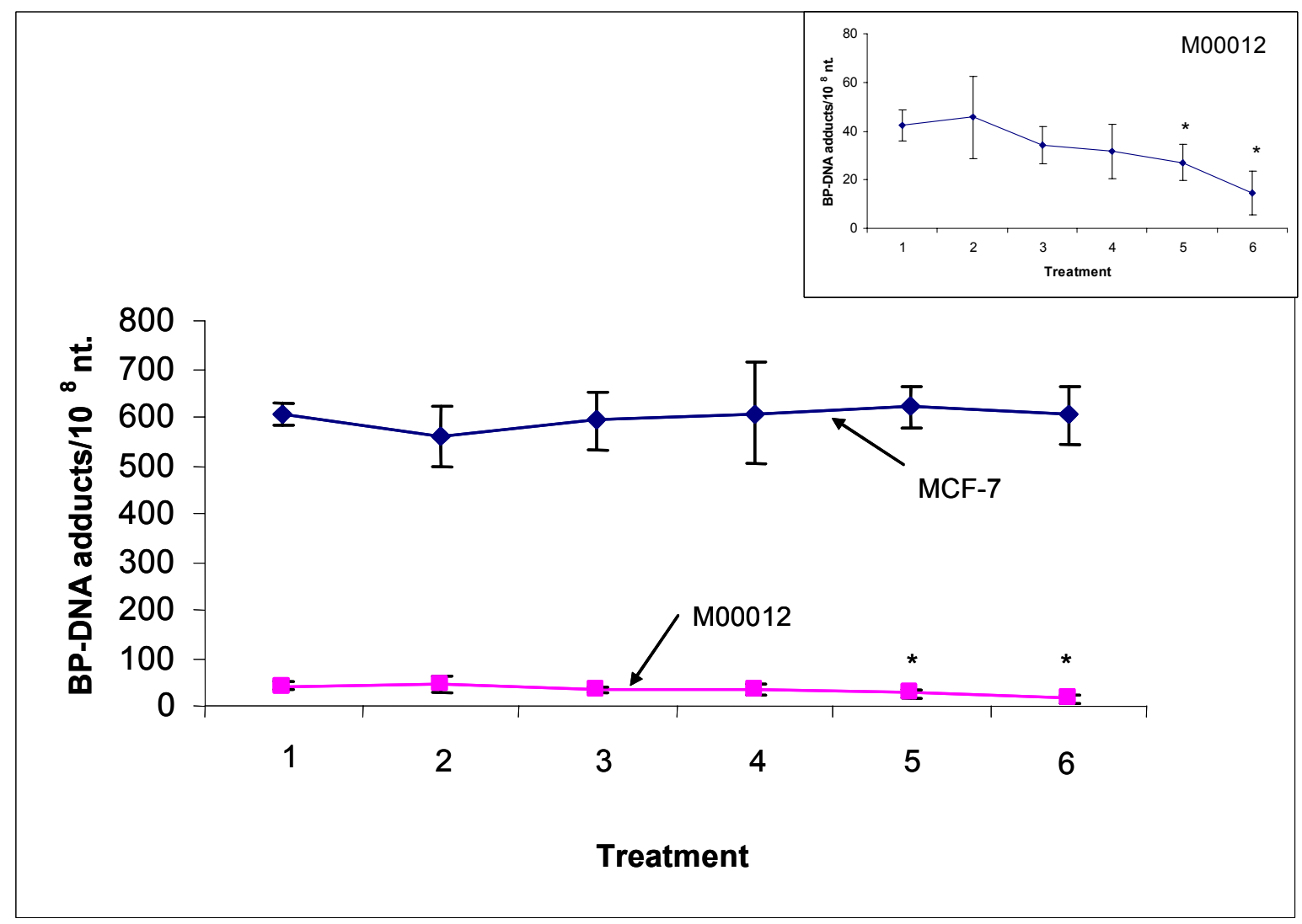

Figure 26: BP-DNA adduct levels $/ 10^{8}$ nucleotides in MCF-7 and M00012 on treatment with $\mathrm{BP}$ in the absence or presence of a range of CHL concentrations. Inset shows a clearer picture of the response pattern of M00012.

* indicates statistically significant reduction compared to cells treated with BP alone Treatments: $1=\mathrm{BP}$ alone, $2=$ Pre $2 \mu \mathrm{M} \mathrm{CHL}+$ post co-treatment, $3=$ Pre $3 \mu \mathrm{M} \mathrm{CHL}+$ post co-treatment, $4=$ Pre $4 \mu \mathrm{M} \mathrm{CHL}+$ post co-treatment, $5=$ Pre $8 \mu \mathrm{M} \mathrm{CHL}+$ post cotreatment, $6=$ Pre $16 \mu \mathrm{M} \mathrm{CHL}+$ post co-treatment 


\subsection{Correlation of $C Y P 1$ gene expression to BP-DNA adducts}

\subsubsection{Correlation of cell strain origin with $C Y P 1$ gene expression and DNA adduct formation}

To examine the existence of a relationship, if any, between age of the donors and inter-individual variability in the induction of $C Y P 1$ enzymes and DNA adduct formation, a correlation analysis of age versus the $C Y P 1$ expression and DNA adduct levels was carried out across the 20 NHMECs used in the study. There was no correlation between age of the donors and CYP1A1 expression $\left(\mathrm{r}^{2}=0,0.02,0.02\right.$ and 0.07 for $\mathrm{BP}$, co-treatment, pre CHL + post BP treatment and pre CHL + post co-treatment, respectively), CYP1B1 expression $\left(\mathrm{r}^{2}=\right.$ $0.04,0.15,0.13$, and 0.22 for $\mathrm{BP}$, co-treatment, pre $\mathrm{CHL}+$ post $\mathrm{BP}$ treatment and pre $\mathrm{CHL}+$ post co-treatment, respectively) and BP-DNA adduct formation $\left(\mathrm{r}^{2}=0.10,0.11,0.10\right.$ and 0.07 for $\mathrm{BP}$, co-treatment, pre $\mathrm{CHL}+$ post $\mathrm{BP}$ treatment and pre $\mathrm{CHL}+$ post co-treatment, respectively).

\subsubsection{Correlation of CYP1 expression to DNA adducts in 20 NHMECs on treatment with $\mathrm{BP}$ in the absence or presence of $\mathrm{CHL}$}

In order to understand the contribution and relevance of the Ahr mechanism to BP metabolism, a correlation of $C Y P 1 A 1$ and/or $C Y P 1 B 1$ expression to the level of BP-DNA adducts was carried out. Also, the correlation $\left(\mathrm{r}^{2}\right)$ of $C Y P 1 A 1$ expression in the presence of $\mathrm{BP}$ alone, co-treatment, pre $\mathrm{CHL}+$ post $\mathrm{BP}$ treatment and pre $\mathrm{CHL}+$ post co-treatment to the levels of BP-DNA adducts by the corresponding treatments was $0.19,0.16,0.16$ and 0.13 , respectively. Similarly, the correlation $\left(\mathrm{r}^{2}\right)$ of $C Y P 1 B 1$ expression in the presence of BP alone, co-treatment, pre $\mathrm{CHL}+$ post $\mathrm{BP}$ treatment and pre $\mathrm{CHL}+$ post co-treatment to the 
levels of BP-DNA adducts by the corresponding treatments were $0.09,0.03,0.11$ and 0.12 , respectively. Also, since both $C Y P 1 A 1$ and $C Y P 1 B 1$ are involved in the metabolism of $\mathrm{BP}$, a correlation analysis of the extent of CYP1A1 + CYP1B1 expression by BP alone, cotreatment, pre CHL + post BP treatment and pre CHL + post co-treatment to the levels of BP-DNA adducts by the corresponding treatments was also carried out. The correlation $\left(\mathrm{r}^{2}\right)$ CYP1A1 + CYP1B1 expression on treatment with $\mathrm{BP}$ alone, co-treatment, pre $\mathrm{CHL}+$ post $\mathrm{BP}$ treatment and pre CHL + post co-treatment to the levels of BP-DNA adducts by the corresponding treatments were $0.17,0.13,0.18$ and 0.15 , respectively. The basal expression of CYP1B1 was higher than that of CYP1A1.

\subsubsection{Correlation of CYP1 expression to DNA adducts in 2 NHMECs on treatment with}

\section{$B P$ or $B[e] P$ in the absence or presence of $C H L$}

In a comparative study between BP and B[e]P across 2 NHMECs (M98015 and M98025), there was no correlation between BP induced CYP1A1 expression and the level of BP-DNA adducts in the absence and presence of CHL in M98015 $\left(\mathrm{r}^{2}=0.02\right)$. However, there was a very good correlation in M98025 $\left(\mathrm{r}^{2}=0.94\right)$. BP induced CYP1B1 expression in the absence and presence of CHL correlated moderately with the level of BP-DNA adducts in M98015 $\left(\mathrm{r}^{2}=0.47\right)$ and well in M98025 $\left(\mathrm{r}^{2}=0.74\right)$. Correlation of BP induced CYP1A1+ CYP1B1 expression with the level of BP-DNA adducts was poor $\left(\mathrm{r}^{2}=0.20\right)$.

Although $\mathrm{B}[\mathrm{e}] \mathrm{P}$ induced a low level of $C Y P 1$ expression, as mentioned in section 4.3.2 it did not form a detectable level of DNA adducts. 
4.4.4 Correlation of CYP1 gene expression to DNA adducts in MCF-7 and M00012 or 20 NHMECs on treatment with BP in the absence or presence of CHL

Comparison of CYP1 expression with the level of BP-DNA adducts in MCF-7 versus M00012 across different treatments (BP, co-treatment, pre CHL + post BP treatment and pre $\mathrm{CHL}+$ post co-treatment) showed a poor correlation for CYP1A1 expression versus BP-DNA adducts $\left(\mathrm{r}^{2}=0.33\right)$. There was no correlation between $C Y P 1 B 1$ expression versus BP-DNA adducts $\left(\mathrm{r}^{2}=0.06\right)$. The expression of $C Y P 1 A 1+C Y P 1 B 1$ across all treatment groups versus BP-DNA adducts by the corresponding treatments was 0.32 and 0.92 for MCF-7 and M00012, respectively. When compared against all the 20 NHMECs, the correlation $\left(\mathrm{r}^{2}\right)$ of CYP1A1 with BP-DNA adducts and CYP1B1 with BP-DNA adducts across all treatment groups were 0.20 and 0.12 , respectively. The expression of $C Y P 1 A 1+C Y P 1 B 1$ across all treatment groups versus BP-DNA adducts by the corresponding treatments for all 20 NHMECs was 0.21 .

\subsubsection{Correlation of CYP1 gene expression to DNA adducts in MCF-7 and M00012 on treatment with BP in the absence or presence of different CHL concentrations}

The correlation of CYP1A1 expression versus levels of BP-DNA adducts in MCF-7 cells on treatment with BP in the absence or presence of different CHL concentrations was poor $\left(\mathrm{r}^{2}=0.16\right)$ but moderate in M00012 $\left(\mathrm{r}^{2}=0.54\right)$. There was no correlation between $C Y P 1 B 1$ expression versus levels of BP-DNA adducts in both MCF-7 cells $\left(r^{2}=0.02\right)$ and M00012 $\left(r^{2}\right.$ $=0.07)$. The overall correlation of $C Y P 1 A 1+C Y P 1 B 1$ expression to DNA adducts was poor for both MCF-7 $\left(r^{2}=0.16\right)$ and M00012 $\left(r^{2}=0.37\right)$. 


\subsection{DISCUSSION}

\subsection{Gene expression by microarrays}

The development of cancer is a 'multi-hit' process requiring perturbation of a number of normal cellular processes and in turn the function of a multitude of genes that orchestrate these processes. In this study, we observed alterations both in the extent and patterns of expression of many genes belonging to different functional categories, on exposure to BP in the absence or presence of CHL. A complete list of all the genes discussed here can be found in Tables A, B, C, D at (http://www.cdc.gov/niosh/ext-supp-mat/MTP/index.htm).

\subsubsection{Genes altered by BP treatment}

Various members of the Phase I and Phase II battery of enzymes were up-regulated on treatment with BP. The highly consistent increase in the expression of $C Y P 1 B 1$ across all the 6 NHMEC strains may suggest its possible role as the predominant CYP isoform involved in the metabolism of BP to DNA binding electrophiles in most individuals. These results are in agreement with previous studies from our laboratory (45). It also parallels another study which sought to examine 'expression signatures' of tobacco smoke exposure in a population of 85 individuals using peripheral blood leukocytes. In this study, CYP1B1 was found to be one of the genes which consistently and significantly correlated with plasma cotinine concentrations (Lampe et al. 2004). The remaining members of the Phase I cluster of enzymes up-regulated by BP in this study (CYP39A1, FMO, and CYP1A1) are known to be involved in the metabolism of steroids and various endogenous substrates in addition to toxicants. The aldo-keto reductase family 1, member D1 (AKR1D1), an enzyme up-regulated by $\mathrm{BP}$ has been documented to be involved in prevention of oxidative free radical damage 
apart from its role in endogenous aldehyde metabolism (Pappa et al. 2003). Its up-regulation may indicate to its probable role in the scavenging of any free radicals generated by catalytic cycling of BP (Porter et al. 1991). The aldehyde dehydrogenases ALDH1A3 and ALDH3A1 have been reported to be involved in aldehyde detoxification generated from alcohol metabolism and lipid peroxidation.

Given some of the hallmarks of cancer being enhanced uncontrolled cell proliferation and dysregulation of normal cellular controls it may not be surprising that various genes possessing diverse roles in transcriptional regulation, cell signaling, cellular proliferation, cell matrix adhesion and other critical cellular processes may be targets of attack by carcinogens in different individuals. This might explain the alteration in the expression patterns of various genes belonging to these categories. They may also represent a response pattern of the cell to carcinogenic stress as induced by exposure to BP. The up-regulation of genes like the son of sevenless (SOS) (a guanine exchange factor well known for its role in Ras activation), STK4 (a serine threonine kinase up-regulated in certain inflammatory breast cancers (Bieche et al. 2004)), EREG (a member of the epidermal growth factor family found to exhibit enhanced expression in various epithelial tumors (Yamamoto et al. 2004)) strongly point to this possibility. In fact, BP has been shown to be capable transforming and immortalizing cells in vitro (Gudjonsson et al. 2004).

$\mathrm{BP}$ is also an immunotoxicant and has been found to lead to $\mathrm{T}$ cell suppression (Rodriguez et al. 1999) and inhibition of B cell lymphopoiesis (Hardin et al. 1992). Among the battery of immune response genes altered by BP, interleukin 1 beta (IL1B), a proinflammatory cytokine with a proangiogenic role in vivo, was consistently up-regulated in all the 6 cell strains on exposure to BP. This may suggest that among the many changes 
occurring following exposure to $\mathrm{BP}$, one of them could be enhanced cell proliferation and angiogenesis. Also, exposure to BP has been found to affect oxidative damage and immune system injuries probably necessitating up-regulation of inflammatory cytokines such as IL1B as a defense mechanism. This is similar to a study where exposure of rats to $\mathrm{Fe}_{2} \mathrm{O}_{3}$ or $\mathrm{BP}$ or in combination to study their role in lung cancer resulted in enhanced production of various inflammatory cytokines including IL1B (Garcon et al. 2001). Of the other immune response genes up-regulated by BP, MAL, a T cell differentiation proteolipid has been found to behave as a tumor suppressor (Kazemi-Noureini et al. 2004) and also has a role in apoptosis (Kohler et al. 1999). Any BP induced cellular damage may be triggering its up-regulation. Not much is known at this time about the human T-cell leukemia virus enhancer factor (HTLF). SECTM1 (K12), another immune response gene, is protease inhibitor thought to have a role in hematopoiesis and in immunogenic processes by acting as CD7 ligand (SlentzKesler et al. 1998). Though initially unknown, CD7 is now known to be involved in $\mathrm{T}$ and NK cell activation and cytokine production. K12 by interacting with CD7 is, therefore, thought to play a role in regulation of thymocyte signaling and cytokine release (Lam et al. 2005).

Various other genes up-regulated by BP share an unknown association with carcinogenesis. While some are well characterized genes others appear to be mostly ESTs or sequences coding for hypothetical proteins. BEX1 and LBH are genes having a role in developmental regulation being involved in neurogenesis and early limb development, respectively (Briegel et al. 2001; Koo et al. 2005). Recently, BEX1 has been reported to be overexpressed in patients with acute myeloid leukemia (Quentmeier et al. 2005). These 
might provide cues to the manner in which BP manifests some of its developmental toxicities.

Alterations in expression profiles induced by BP appear to be mainly mediated through up-regulation of genes rather than down-regulation given that only one cell strain exhibited down-regulation of genes by SLR $\geq 1.5$.

Only one gene with a potential role in immune responses (CXCL14) was downregulated by BP. Contradictory reports exist regarding the role of CXCL14 (BRAK) with some studies pointing more to a role of a tumor suppressor inhibiting tumor growth (Schwarze et al. 2002; Shellenberger et al. 2004) and yet others reporting a role for it in enhanced proliferation, migration, and invasion of myoepithelial cells and myofibroblasts (Allinen et al. 2004).

Taken together, there appears to be a dual role of BP by either enhancing (in most cases) the expression of genes which may aid in the various aspects of carcinogenesis or in parallel also modulating the expression of genes which may serve to have a protective effect by maintaining cellular integrity. Considerable inter-individual variations in response patterns are also apparent with most genes not being consistently altered in all individuals except $C Y P 1 B 1$.

\subsubsection{Genes altered by Pre CHL + co-treatment}

CHL has been documented to modulate various aspects of carcinogenesis and mutagenesis either through non-specific inhibition of CYPs (Waters et al. 1996), upregulation of various members of the detoxification battery (Singh et al. 1996; Fahey et al. 2005), enhancement of apoptosis (Chiu et al. 2005), modulation of carcinogen transport 
(Mata et al. 2004), mitigation of oxidative stress (Kumar et al. 2004) or various combinations of these. Alteration in the expression profiles of genes belonging to various functional categories as seen in this study may point to additional targets of CHL. The large number of genes modulated by pre CHL + co-treatment may be due a 'synergistic interaction' between $\mathrm{BP}$ and CHL.

CHL modulated similar functional classes of genes as those altered by BP though some of the individual members within each functional class were different. Most members of the Phase I group of enzymes are common to BP treated cells indicating that their expression may predominantly be affected by carcinogenic stress (BP) or the fact that CHL may be a 'bifunctional inducer' inducing Phase I enzymes too in addition to Phase II enzymes or both. Again, only $C Y P 1 B 1$ was consistently up-regulated by SLR $\geq 1.5$ (except M99005) in all the donors while considerable inter-individual variations existed in expression patterns of the other genes. Several other members of the aldehyde dehydrogenase and aldo-keto reductase (AKR1) families, apart from the ones altered by BP treatment were up-regulated by CHL compared to cells treated with BP alone further indicating a 'bifunctional' role of CHL. Most of these members were also up-regulated in a consistent fashion though not by SLR $\geq 1.5$ in all 6 donors. Many members of the AKR1 family have been implicated for their role in the carcinogenesis of organs such as the breast and endometrium by playing a vital role in progesterone signaling and in turn also regulating the estrogen: progesterone balance (Bauman et al. 2004; Ji et al. 2004).

Various other genes involved in phenomena as diverse as cell cycle control, apoptosis and developmental regulation were differentially modulated in different individuals. A multitude of genes coding for proteins such as kinases, Ca binding proteins, cell junction 
proteins, growth factors, G proteins, various receptors, ligands and other regulatory proteins involved in various facets of cell signaling were differentially regulated by CHL being 'up' in some donors and 'down' in others thereby indicating that modulatory effects of CHL may partly be mediated through modulation of cellular communication processes.

Various immune response genes were also altered differentially by CHL. Genes such as SLP1, a serine protease (Devoogdt et al. 2003), interleukin 8 (Huang et al. 2002; Mian et al. 2003), IL1B and several other altered immune response genes have been found to enhance the risk of different cancers. Their up-regulation by CHL treatment may be explained as the response possibly mediated by the interactive action of $\mathrm{BP}+\mathrm{CHL}$ together. Interferon alpha (IFNA), a gene with the highest degree of up-regulation compared to all other genes (in one cell strain M99016) has at times been shown to exhibit antitumor effects (Zella et al. 1999). Its differential regulation in the 6 cell strains could be due to polymorphisms in the gene for this cytokine or other down stream modulators in the signaling pathway thereby ultimately leading to differential expression extents and differential effects. CD80 didn't exhibit upregulation in most of the cell strains except M99016 and has been reported to activate cytotoxic T lymphocytes (Chan et al. 2004) and also provide negative signals for the proliferation and IgG secretion of normal B cells and B cell lymphomas (Suvas et al. 2002). CD86 up-regulated in most of the cell strains has been found to have a role in priming and activation of naive and memory T cells, respectively (Jeannin et al. 2000) and also for enhanced activity of B cells (Suvas et al. 2002). PI3, protease inhibitor, up-regulated by CHL treatment in most of the individuals (except M99005 and M99025) has been reported to have a role in cell differentiation and apoptosis (Yamamoto et al. 1997) and possibly in the control of tumor cell invasion (Alkemade et al. 1993). 
A number of other genes with diverse functions, ORF's and sequences coding for hypothetical proteins were differentially modulated by CHL and are presently not very well characterized in relation to cancer. However, many of these are involved in several developmental processes indicating that developmental processes may also form some key targets of attack several by some PAHs.

Genes down-regulated by the interactive action of BP + CHL included those whose enhanced expression has been documented to aid either directly or indirectly the progression of various cancers, e.g.:SOD2 (Drane et al. 2001), CRY61 (Lin et al. 2005), RAI3 (Wu et al. 2005) and several others. However, various other genes whose enhanced expression is known to inhibit various facets of carcinogenesis were also down-regulated by the interactive action of BP + CHL. These included AFT3 (Yan et al. 2005), OCLN (Tobioka et al. 2004), GADD45B (Qiu et al. 2003), ADAMST1 (Iruela-Arispe et al. 2003), MEST1 (Pedersen et al. 1999) and DOC1 (Schwarze et al. 2002).

Nine genes with a role in immune responses were down- regulated by BP + CHL. Of these, some like the interferon alpha inducible protein (IFI27) down-regulated in all 6 cell strains, is a protein suggested to be a novel biomarker of epithelial proliferation and cancer (Suomela et al. 2004). GIP2 (INSIG1) consistently down-regulated in all the cell strains has previously been suggested to function intracellularly as a ubiquitin homologue and a cytokine that induces production of IFN-gamma to augment NK/lymphokine-activated killer cell proliferation and function, possibly playing a vital role in antiviral response (Zhao et al. 2005). CXCL2 has been implicated in inflammatory responses along with other chemokines and cytokines (Yamagami et al. 2003) in human corneal endothelium and human melanoma cells (Gallagher et al. 2005). 
Not much is known about the role of the remaining genes in relation to cancer and disease.

\subsection{Correlation of gene expression on microarrays versus RT-PCR}

Overall, there was a good correlation between the magnitude of expression for different genes as measured by GC and by RT-PCR. However, there were a few genes which correlated only moderately to poorly. In general, RT-PCR was more sensitive than GC in measuring gene expression changes. The lack of good correlation for some genes may be due to the comparatively lower sensitivity of GC in relation to RT-PCR in addition to differences in the exact regions being probed by GC and RT-PCR. A low level of expression of particular gene in only some individuals may cause GC to 'miss' it or at most exhibit its expression at an extremely low level, whereas RT-PCR being more sensitive may be able to more realistically track its expression giving a higher FC compared to GC culminating in a poor correlation.

CHL clearly appears to be a 'bifunctional inducer' modulating the expression of both bioactivating and detoxication enzymes. Various other functional categories of genes modulated by the interactive action of $\mathrm{BP}+\mathrm{CHL}$ may point to various other presently unknown targets and mechanisms of action of CHL though these will need to be confirmed. Some genes (AKR1C1, SECTM1, CRY61 and a few others) following a similar trend of modulation in most donors though not necessarily by SLR $\geq 1.5$ in all of them suggest that these could be good putative 'biomarkers of exposure' to be confirmed by further validation. Many other genes not consistently altered in a similar direction across all donors suggest the presence of extensive inter-individual variability in response to carcinogenic stress and 
intervention strategies, an issue addressed earlier in our laboratory regarding CYPIAI and CYP1B1 (John et al.). However, despite inter-individual differences, CYPIB1 followed by $A L D H 1 A 3$ were consistently up-regulated in most individuals following treatment with $\mathrm{BP}$ as well as $\mathrm{BP}+\mathrm{CHL}$ indicating that these could be definite biomarkers of $\mathrm{BP}$ exposure. The consistent up-regulation of CYP $1 B 1$ by BP has been previously reported from our laboratory (Keshava et al. 2005) and was further confirmed by RT-PCR studies of the 20 NHMEC strains as discussed below.

\subsection{Genetic damage in terms of CYP1 induction and DNA adduct formation in 20 NHMECs on treatment with BP in the absence or presence of CHL}

PAHs, including BP, are known to bring about their own metabolism through the Ahr pathway (Ma et al. 2003). CYP1A1, a substrate inducible enzyme expressed predominantly in extra hepatic tissues including the breast (Whitlock 1999; Masson et al. 2005) was highly induced in our studies ( 96 fold variation). Its induction, however, correlated poorly with donor age. This is in agreement with another study which also found an age independent variation in the expression of CYP1A1 in normal breast tissue samples $(\mathrm{n}=58)$ obtained from breast cancer patients and normal cancer free individuals (Goth-Goldstein et al. 2000). The induction of CYP1B1 was much less (43 fold variation) as compared to CYPIA1 and also correlated poorly with the age of the donor. In contrast to CYP1A1, CYP1B1 is expressed constitutively in various extra hepatic tissues including the breast but exhibits weak induction by Ahr ligands which may explain its lower induction in our study compared to CYP1A1 (Ma et al. 2003). 
The wide inter-individual differences among the cell strains in the induction of both $C Y P 1 A 1$ and $C Y P 1 B 1$ could be explained at least in part due to polymorphisms in the genes coding for CYPs and other Phase I enzymes, AhR-Arnt as well as detoxication and repair enzymes (Ma et al. 2003; Weston et al. 2005). In addition, physiological factors (such as the presence of endogenous agonists or antagonists), environmental and dietary exposures of the donors could also play a role although these are much less likely, since the cells have been in culture for long. Given the fact that majority of the cell strains were of Caucasian origin, variability due to ethnic differences though low cannot be ruled out completely. The presence of only a moderate to poor correlation between the induction of both $C Y P 1 A 1$ and $C Y P 1 B 1$ both in the absence and presence of CHL may suggest that both of these isoforms could be differentially regulated in response to $\mathrm{BP}$ or the fact that one isoform may be involved to a greater extent in carcinogen metabolism than the other or both. In fact, $C Y P 1 B 1$ has been found to be catalytically more active than $C Y P 1 A 1$ in the bioactivation of different PAHs (Shimada et al. 1996).

The universal reduction in the induction of both $C Y P 1 A 1$ and $C Y P 1 B 1$ in all the cell strains upon co-treatment as well as the existence of a good correlation between the fold induction upon treatment with BP alone and upon co-treatment (when CHL is present along with the carcinogen) suggests the action of CHL as an 'interceptor molecule,' reducing the availability of BP to trigger the Ahr pathway. Other in vivo studies have also found highly effective inhibition when CHL was co-administered with the carcinogen (Dashwood 1997a). A reduction in $C Y P 1$ expression in most individuals upon treatment with CHL prior to carcinogen exposure (pre $\mathrm{CHL}+$ post $\mathrm{BP}$ ) suggests mitigation of $C Y P 1$ expression by mechanisms other than just carcinogen sequestration. Prior exposure to CHL may in some 
manner alter specific cell signaling circuitry or modulate the abundance of Phase I enzymes directly or indirectly by altering the genes/proteins involved in the 'Ahr' battery.' This however, might not be a universal phenomenon and the 'bifunctional' nature of CHL may be coming into play to different extents in different individuals which might in part explain the increase in $C Y P 1$ expression in some individuals upon CHL pretreatment. However, the exact molecular mechanism of CHL mediated modulation of CYPI gene expression upon its administration prior to carcinogen exposure is currently unknown. Pre CHL treatment followed by co-treatment enables CHL to act both as a chelator and as a modulator of CYP1 gene expression thereby causing a reduction in $C Y P 1$ expression in the majority of individuals and also causing a greater reduction in expression compared to other CHL treatments where CHL acts predominantly by any one of the above mechanisms. This is exemplified by the presence of a good correlation between the percentage reduction in the expression of CYP1A1 to CYPIB1 upon pre-treatment with CHL followed by co-treatment in the absence of outliers.

The reduction in adduct levels in most of the cell strains on co-treatment again points to the role of CHL as an 'interceptor' molecule sequestering the carcinogen and preventing it from interacting with DNA. This mechanism, however, requires CHL to be present in large molar excess compared to the carcinogen (Dashwood 1997a). Non-specific inhibition of CYPs may also lead to reduced metabolism of the carcinogen in turn leading to reduced availability of the carcinogen to interact with DNA as in a pre CHL + post BP treatment. However, the poor correlation between CYP1 expression and DNA-adduct levels may suggest interplay of Ahr independent mechanisms of activation along with cross-talks with various other cellular pathways and/or factors. Aldo keto reductases (AKRs) have also been 
shown to have a role in the metabolism of BP. In this respect, human AKR1A1, AKR1C1AKR1C4 have been found to be particularly active in metabolizing BP to reactive quinone metabolites and also generating of reactive oxygen species. AKR1A1 has been found to serve a dual role in directly metabolizing the BP-7,8-diols to reactive quinones and also indirectly trans-activating CYP1B1 (Jiang et al. 2004). A peroxidase pathway for BP metabolism is also documented to exist (Cavalieri et al. 1995). Though there are hardly any studies on the role of flavin monooxygenases in BP metabolism it may not be misleading to assume that these monooxygenases along with various other Phase I enzymes may potentially play at least a minor role in the metabolism of BP in some individuals. Many of these processes compete with the CYP pathway for BP metabolism. In addition, basal expression along with induced CYPs is responsible for metabolism of the compound to toxic metabolites. Therefore, trying to correlate only induced expression with the extent of BPDNA adducts may give poor correlation. The basal expression levels in terms of RNA copy number need to be determined in future studies. Also, should CHL be triggering Phase II metabolic reactions (Fahey et al. 2005) or DNA repair process some of the reactive metabolites/ adducts formed may either be prevented from interacting with DNA or repaired, respectively leading to the lack of a good correlation.

\subsection{Genetic damage in terms of CYP1 induction and DNA adduct formation in 2}

\section{NHMECs on treatment with $B P$ or $B[e] P$ in the absence or presence of $C H L$}

With respect to the studies on comparison of $\mathrm{BP}$ versus $\mathrm{B}[\mathrm{e}] \mathrm{P}$, the lower potency of $\mathrm{B}[\mathrm{e}] \mathrm{P}$ in inducing CYPs and forming adducts was apparent. $\mathrm{BP}$ is a strong $\mathrm{PAH}$ receptor (8S) ligand but a weak PAH-binding protein (4S) ligand whereas $\mathrm{B}[\mathrm{e}] \mathrm{P}$ is mainly a $4 \mathrm{~S}$ ligand 
but a poor AH receptor ligand (Houser et al. 1992; Sterling et al. 1994). This explains the overall poor induction of $C Y P 1$ by $\mathrm{B}[\mathrm{e}] \mathrm{P}$ compared to $\mathrm{BP}$ in both cell strains. However, an explanation for the differential action of CHL towards $\mathrm{B}[\mathrm{e}] \mathrm{P}$ induced $C Y P 1$ expression is unknown. The enhancement in CYPl expression in most cases involving a co-treatment of CHL with the carcinogen may suggest a lack of efficient complex formation between $\mathrm{B}[\mathrm{e}] \mathrm{P}$ and CHL probably due to some change in carcinogen structure and subsequent alteration of stabilizing forces. In fact CHL has been found to form tight molecular complexes with planar aromatic hydrocarbons possessing at least partial ring structure when present in large molar excess to the carcinogen and administered simultaneously with the carcinogen (Dashwood 1997a). CHL in low concentrations has been shown to have the potential to act as an NADPH generating system by replacing glucose 6-phosphate (Waters et al. 1996). This was a mechanism suggested for the 'potentiator effect' of low concentration of CHL against nitrosamines (Waters et al. 1996) which was, however, reversed at higher CHL concentrations. Here, though the concentration of CHL used for intervention of BP and B[e]P was the same, in the absence of effective complex formation as in the case of $\mathrm{B}[\mathrm{e}] \mathrm{P}$, the low concentration of free CHL may have the potential to bring about 'potentiation' through the above mechanism. This suggests that though some carcinogens may be only weakly carcinogenic they may be rendered more active in the presence of certain chemopreventive agents which are generally administered to mitigate the ill effects of more potent carcinogens depending on the concentration and treatment protocol involved. The mechanisms of BPDNA adduct reduction by CHL may occur through some of the above discussed mechanisms. However, the lack of any detectable adducts by $\mathrm{B}[\mathrm{e}] \mathrm{P}$ in the absence or presence of CHL, suggests that there may be a very low level of $\mathrm{B}[\mathrm{e}] \mathrm{P}-\mathrm{DNA}$ adducts given 
its much lower carcinogenicity compared to BP. In addition, synchronous fluorescence spectrometry (SFS) may not be sensitive enough to detect rather low levels of adducts formed by $\mathrm{B}[\mathrm{e}] \mathrm{P}$ (detection limit of 1 in $10^{7}$ adducts). A more sensitive assay will be needed to detect these low levels of adducts. The presence of a good correlation between CYPI gene expression and BP-DNA adduct formation in M98025 but only a moderate to poor correlation in M98015 again points to wide inter-individual variation in induction and response to intervention.

\subsection{Genetic damage in terms of CYP1 induction and DNA adduct formation in MCF-7 cells versus an NHMEC (M00012) on treatment with $\mathrm{BP}$ or $\mathrm{B}[\mathrm{e}] \mathrm{P}$ in the absence or presence of CHL}

Cancerous cells are often used to study various aspects of carcinogenesis. Their relative ease of maintenance and faster generation time are some factors which favor their use. Here, MCF-7, an estrogen receptor positive breast cancer cell line used in comparison with normal breast cells exhibited a response pattern sharply different from those of normal cells. CHL failed to mitigate BP induced CYP1 expression and DNA adduct across a range of CHL treatments and CHL concentrations suggesting that CHL neither behaves as a CYP inhibitor nor an interceptor molecule in these cells. However, it remains to be determined whether this is an exclusive response exhibited by MCF-7 cells or is commonly exhibited by other cancerous cells including other breast cancer cells, some of which may be estrogen receptor negative. Though the induction of CYP1B1 was low compared to CYP1A1 its basal expression was much higher than that of CYP1A1. This has been shown even in a few other studies (McKay et al. 1995; Spink et al. 1998). Nevertheless, it shows that cancerous cells 
exhibit different response patterns compared to normal cells and therefore cannot be used as surrogates to predict responses of normal cells to carcinogens and chemopreventive agents. BP is a widely distributed environmental carcinogen and a high risk factor for a large section of the world's population whose dietary lifestyle involves use of tobacco. Smoking alone has been linked to about $30 \%$ of all cancer deaths and more than 400,000 premature deaths/year in the U.S. alone (http://www.cancer.org/docroot/PED/ped_10.asp; http://www.cdc.gov/HealthyYouth/tobacco). A greater understanding of the 'molecular changes' that ensue following PAH exposure can help better design exposure monitoring and intervention strategies. This study has revealed at least a few potential biomarkers of exposure some of which could be potential targets for intervention strategies. Clearly, CYP1B1 is one such target as seen from this study. Additionally, CHL appears to function as a bifunctional inducer differentially modulating the expression of most genes except a few where most individuals seem to respond in a similar fashion. Wide inter-individual variations will need to be accounted for during biomarker validation as well as while designing intervention strategies. 


\subsection{SUMMARY AND CONCLUSIONS}

These studies were intended to extend our understanding of human chemical carcinogenesis, potential biomarkers of carcinogen exposure and strategies for chemoprevention. To do this a human mammary cell model system was exposed in vitro to benzo[a]pyrene a known polycyclic aromatic hydrocarbon carcinogen, with or without chlorophyllin, a potential chemopreventive agent. The effects of these exposures on gene expression and carcinogenesis precursor events were evaluated.

Global gene expression analysis was carried out to seek for biomarkers of carcinogen exposure and chemopreventive intervention. Immune response genes altered on exposure to polycyclic aromatic hydrocarbons, with or without the modulating agent, chlorophyllin, were analyzed in the context of having cross-talks between themselves as well as interacting with several other cellular pathways. The expression of cytochrome P450 (CYP1) enzymes involved in polycyclic aromatic hydrocarbon metabolism was examined across a spectrum of individuals in relation to their ability to cause DNA damage, in the absence and presence of chlorophyllin.

CYP1B1 appeared to be a potential biomarker of polycyclic aromatic hydrocarbon exposure. Chlorophyllin served as an effective chemopreventive agent in mitigating genetic damage by reducing polycyclic aromatic hydrocarbon metabolism and the extent of DNA damage.

The main conclusions from the study are that:

- Genes from a broad spectrum of functional categories were altered by exposure to BP in the absence or presence of chlorophyllin. 
- Only CYPIB1 was altered in a consistent manner across all donors, and therefore appeared to be good biomarker of polycyclic aromatic hydrocarbon exposure and chemopreventive intervention. All other genes were altered in a less consistent in one or more donors indicating inter-individual variations in responses.

- Extensive inter-individual variations were observed in the extent of induction and modulation of CYP1A1 and CYP1B1 expression as well as benzo[a]pyrene-DNA adduct formation on exposure to benzo[a]pyrene in the absence or presence of chlorophyllin.

- The reduction of CYPI gene expression and BP-DNA adduct formation in most normal human mammary epithelial cells suggests the role of chlorophyllin acts as a ‘cytochrome P450 inhibitor' as well as a 'chelator' in mitigating genetic damage.

- Chlorophyllin may enhance the carcinogenicity of even weakly carcinogenic polycyclic aromatic hydrocarbons. The extent of enhancement depends on the nature of the carcinogen as well as the time of chlorophyllin administration as seen from the enhancement of benzo[e]pyrene induced CYP1 gene expression by pre chlorophyllin + post benzo[e]pyrene treatment but not any of the other chlorophyllin treatments.

- Cancerous MCF-7 cells exhibited high levels of CYP1A1 induction and benzo[a]pyrene-DNA adduct formation on exposure to benzo[a]pyrene, both in the absence and presence of chlorophyllin, suggesting probably a different mechanism of chlorophyllin action in cancerous cell as opposed to normal cells and/or the fact that MCF-7 may be an inappropriate surrogate to normal mammary cells.

- Only chlorophyllin concentrations higher than those used in other experiments $(8 \mu \mathrm{M}$, $16 \mu \mathrm{M})$ mitigated BP induced CYP1 gene expression in MCF-7 cells. However, none 
of the chlorophyllin concentrations mitigated BP-DNA adduct formation in MCF-7 cells supporting the previous conclusion.

- The lack of reduction in the extent of CYP1 gene expression and benzo[a]pyrene-DNA adduct formation in MCF-7 cells suggests that chlorophyllin performs poorly as a 'CYP inhibitor' and 'chelator' in MCF-7 cells. 


\subsection{FUTURE DIRECTIONS}

- In order to gain a better understanding of the correlation between CYP1 gene expression and BP-DNA adduct formation an assessment of gene copy number needs to be determined. This will also help reveal the possible interplay of Ahr independent mechanisms in the metabolism of BP.

- The balance between toxicity and detoxication can be better understood if the effects of CHL on other phase I enzymes, e.g., other monooxygenases, in addition to the phase II enzyme battery are determined.

- The effect of chlorophyllin alone on global gene expression would be a valuable asset to the scientific community involved in screening for new cancer chemopreventive agents.

- Studies on functional CYP1 enzyme abundance and activity following exposure of cells to BP in the absence or presence of CHL will help reveal whether CHL mediated changes are transcriptionally or translationally regulated and in turn will help gain a better understanding of the mechanistic aspects of CHL action and BP metabolism.

- Studies on a wider spectrum of cancerous cells will help determine whether the large extent of genetic damage as seen in MCF-7 cells (as opposed to NHMECs) is a common feature of most cancerous cells and in turn point to the feasibility of using cancerous cells as surrogates to normal cells for studying the carcinogenic process. 


\subsection{REFERENCES}

1. Adamson, R.H., 1989. Induction of hepatocellular carcinoma in nonhuman primates by chemical carcinogens. Cancer Detect Prev 14, 215-219.

2. Affymetrix, 2001. Data Mining Tool User's Manual, version 3.0. Santa Clara, CA, Affymetrix.

3. Affymetrix, 2004. Gene Chip Expression Analysis Technical Manual, Santa Clara, CA, Affymetrix.

4. Alkemade, H.A., Molhuizen, H.O., van Vlijmen-Willems, I.M., van Haelst, U.J., Schalkwijk, J., 1993. Differential expression of SKALP/Elafin in human epidermal tumors. Am J Pathol 143, 1679-1687.

5. Allinen, M., Beroukhim, R., Cai, L., Brennan, C., Lahti-Domenici, J., Huang, H., Porter, D., Hu, M., Chin, L., Richardson, A., Schnitt, S., Sellers, W.R., Polyak, K., 2004. Molecular characterization of the tumor microenvironment in breast cancer. Cancer Cell 6, 17-32.

6. Arimoto, S., Fukuoka, S., Itome, C., Nakano, H., Rai, H., Hayatsu, H., 1993. Binding of polycyclic planar mutagens to chlorophyllin resulting in inhibition of the mutagenic activity. Mutat Res 287, 293-305.

7. Bauman, D.R., Steckelbroeck, S., Penning, T.M., 2004. The roles of aldo-keto reductases in steroid hormone action. Drug News Perspect 17, 563-578.

8. Bez, G.C., Jordao, B.Q., Vicentini, V.E., Mantovani, M.S., 2001. Investigation of genotoxic and antigenotoxic activities of chlorophylls and chlorophyllin in cultured V79 cells. Mutat Res 497, 139-145.

9. $\quad$ Bieche, I., Lerebours, F., Tozlu, S., Espie, M., Marty, M., Lidereau, R., 2004. Molecular profiling of inflammatory breast cancer: identification of a poor-prognosis gene expression signature. Clin Cancer Res 10, 6789-6795.

10. Briegel, K.J., Joyner, A.L., 2001. Identification and characterization of Lbh, a novel conserved nuclear protein expressed during early limb and heart development. Dev Biol 233, 291-304.

11. Brookes, P., Lawley, P.D., 1964. Evidence for the Binding of Polynuclear Aromatic Hydrocarbons to the Nucleic Acids of Mouse Skin: Relation between Carcinogenic Power of Hydrocarbons and Their Binding to Deoxyribonucleic Acid. Nature 202, 781-784.

12. Cavalieri, E.L., Rogan, E.G., 1995. Central role of radical cations in metabolic activation of polycyclic aromatic hydrocarbons. Xenobiotica 25, 677-688.

13. Cavalieri, E.L., Rogan, E.G., 2002. A unified mechanism in the initiation of cancer. Ann N Y Acad Sci 959, 341-354.

14. Cavalieri, E.L., Stack, D.E., Devanesan, P.D., Todorovic, R., Dwivedy, I., Higginbotham, S., Johansson, S.L., Patil, K.D., Gross, M.L., Gooden, J.K., Ramanathan, R., Cerny, R.L., Rogan, E.G., 1997. Molecular origin of cancer: catechol estrogen-3,4-quinones as endogenous tumor initiators. Proc Natl Acad Sci U S A 94, 10937-10942.

15. Chan, R.C., Xie, Y., 2004. CD80 transfected human hepatocellular carcinoma cells activate cytotoxic T lymphocytes to target HCC cells with shared tumor antigens. Oncol Rep 12, 435-442. 
16. Chiu, L.C., Kong, C.K., Ooi, V.E., 2005. The chlorophyllin-induced cell cycle arrest and apoptosis in human breast cancer MCF-7 cells is associated with ERK deactivation and Cyclin D1 depletion. Int J Mol Med 16, 735-740.

17. Chowbay, B., Zhou, S., Lee, E.J., 2005. An interethnic comparison of polymorphisms of the genes encoding drug-metabolizing enzymes and drug transporters: experience in Singapore. Drug Metab Rev 37, 327-378.

18. Consortium, T.I.H., 2003. The International HapMap Project. Nature 426, 789-796.

19. Dashwood, R., Yamane, S., Larsen, R., 1996. Study of the forces of stabilizing complexes between chlorophylls and heterocyclic amine mutagens. Environ Mol Mutagen 27, 211-218.

20. Dashwood, R.H., 1997a. Chlorophylls as anticarcinogens (Review). Int. J. Oncol. 10, 721-727.

21. Dashwood, R.H., 1997b. The importance of using pure chemicals in (anti) mutagenicity studies: chlorophyllin as a case in point. Mutat Res 381, 283-286.

22. Dashwood, R.H., Breinholt, V., Bailey, G.S., 1991. Chemopreventive properties of chlorophyllin: inhibition of aflatoxin B1 (AFB1)-DNA binding in vivo and antimutagenic activity against AFB1 and two heterocyclic amines in the Salmonella mutagenicity assay. Carcinogenesis 12, 939-942.

23. Dashwood, R.H., Xu, M., Orner, G.A., Horio, D.T., 2001. Colonic cell proliferation, apoptosis and aberrant crypt foci development in rats given 2-amino-3-methylimidaz. Eur J Cancer Prev 10, 139-145.

24. de Vogel, J., Jonker-Termont, D.S., Katan, M.B., van der Meer, R., 2005. Natural chlorophyll but not chlorophyllin prevents heme-induced cytotoxic and hyperproliferative effects in rat colon. . J Nutr. 135, 1995 - 2000.

25. Devoogdt, N., Hassanzadeh Ghassabeh, G., Zhang, J., Brys, L., De Baetselier, P., Revets, H., 2003. Secretory leukocyte protease inhibitor promotes the tumorigenic and metastatic potential of cancer cells. Proc Natl Acad Sci U S A 100, 5778-5782.

26. Dhir, H., 1989 Plant extract as desmutagens: some aspects. Acta Bot. Indica. 17 1-11.

27. Diaz, G.D., Li, Q., Dashwood, R.H., 2003. Caspase-8 and apoptosis-inducing factor mediate a cytochrome c-independent pathway of apoptosis in human colon cancer cells induced by the dietary phytochemical chlorophyllin. Cancer Res 63, 1254-1261.

28. Dipple, A., 1995. DNA adducts of chemical carcinogens. Carcinogenesis 16, 437441.

29. Dipple, A., Moschel, R.C., Bigger, C.A.H., 1984. Polynuclear Aromatic Carcinogens. In Chemical Carcinogens. Searle,C.E. (Ed) Vol.1.

30. Drane, P., Bravard, A., Bouvard, V., May, E., 2001. Reciprocal down-regulation of p53 and SOD2 gene expression-implication in p53 mediated apoptosis. Oncogene 20, 430-439.

31. Egner, P.A., Wang, J.B., Zhu, Y.R., Zhang, B.C., Wu, Y., Zhang, Q.N., Qian, G.S., Kuang, S.Y., Gange, S.J., Jacobson, L.P., Helzlsouer, K.J., Bailey, G.S., Groopman, J.D., Kensler, T.W., 2001. Chlorophyllin intervention reduces aflatoxin-DNA adducts in individuals at high risk for liver cancer. Proc Natl Acad Sci U S A 98, 1460114606.

32. Fahey, J.W., Stephenson, K.K., Dinkova-Kostova, A.T., Egner, P.A., Kensler, T.W., Talalay, P., 2005. Chlorophyll, chlorophyllin and related tetrapyrroles are significant inducers of mammalian phase 2 cytoprotective genes. Carcinogenesis 26, 1247-1255. 
33. Gallagher, P.G., Bao, Y., Prorock, A., Zigrino, P., Nischt, R., Politi, V., Mauch, C., Dragulev, B., Fox, J.W., 2005. Gene expression profiling reveals cross-talk between melanoma and fibroblasts: implications for host-tumor interactions in metastasis. Cancer Res 65, 4134-4146.

34. Garcon, G., Gosset, P., Garry, S., Marez, T., Hannothiaux, M.H., Shirali, P., 2001. Pulmonary induction of proinflammatory mediators following the rat exposure to benzo(a)pyrene-coated onto Fe2O3 particles. Toxicol Lett 121, 107-117.

35. Gelboin, H.V., 1980. Benzo[alpha]pyrene metabolism, activation and carcinogenesis: role and regulation of mixed-function oxidases and related enzymes. Physiol Rev 60, 1107-1166.

36. Goldman, R., Shields, P.G., 2003. Food mutagens. J Nutr 133 Suppl 3, 965S-973S.

37. Goth-Goldstein, R., Stampfer, M.R., Erdmann, C.A., Russell, M., 2000. Interindividual variation in CYP1A1 expression in breast tissue and the role of genetic polymorphism. Carcinogenesis 21, 2119-2122.

38. Green, J., Banks, E., Berrington, A., Darby, S., Deo, H., Newton, R., 2000. Nacetyltransferase 2 and bladder cancer: an overview and consideration of the evidence for gene-environment interaction. Br J Cancer 83, 412-417.

39. Gudjonsson, T., Villadsen, R., Ronnov-Jessen, L., Petersen, O.W., 2004.

Immortalization protocols used in cell culture models of human breast morphogenesis. Cell Mol Life Sci 61, 2523-2534.

40. Guengerich, F.P., 1991. Reactions and significance of cytochrome P-450 enzymes. J Biol Chem 266, 10019-10022.

41. Guengerich, F.P., 2001. Common and uncommon cytochrome P450 reactions related to metabolism and chemical toxicity. Chem Res Toxicol 14, 611-650.

42. Guillen, M.D., Sopelana, P., 2003. Polycyclic aromatic hydrocarbons in diverse foods. In Food Safety: Contaminants and toxicants, ed.J.P.F.D’Mello, 175-197.CAB International, Wallingford,Oxon,UK.

43. Hardin, J.A., Hinoshita, F., Sherr, D.H., 1992. Mechanisms by which benzo[a]pyrene, an environmental carcinogen, suppresses B cell lymphopoiesis. Toxicol Appl Pharmacol 117, 155-164.

44. Hayatsu, H., 1995. Complex formation of heterocyclic amines with porphyrins: its use in detection and prevention. Princess Takamatsu Symp 23, 172-180.

45. Hirschhorn, J.N., Lohmueller, K., Byrne, E., Hirschhorn, K., 2002. A comprehensive review of genetic association studies. Genet Med 4, 45-61.

46. Hooven, L.A., Mahadevan, B., Keshava, C., Johns, C., Pereira, C., Desai, D., Amin, S., Weston, A., Baird, W.M., 2005. Effects of suberoylanilide hydroxamic acid and trichostatin A on induction of cytochrome P450 enzymes and benzo[a]pyrene DNA adduct formation in human cells. Bioorg Med Chem Lett 15, 1283-1287.

47. Houser, W.H., Raha, A., Vickers, M., 1992. Induction of CYP1A1 gene expression in H4-II-E rat hepatoma cells by benzo[e]pyrene. Mol Carcinog 5, 232-237.

48. http://drnelson.utmem.edu/CytochromeP450.html.

49. http://medicine.iupui.edu/flockhart/.

50. http://rana.lbl.gov/EisenSoftware.htm.

51. http://www.ariadnegenomics.com.

52. http://www.cancer.gov/cancertopics/factsheet/Prevention/chemoprevention.

53. http://www.cancer.org. 
54. http://www.cancer.org/docroot/PED/ped 10.asp.

55. http://www.cdc.gov/HealthyYouth/tobacco.

56. http://www.cdc.gov/niosh/ext-supp-mat/IR Genes/index.htm.

57. http://www.cdc.gov/niosh/ext-supp-mat/MTP/index.htm.

58. $\quad$ http://www.imm.ki.se/CYPalleles/.

59. http://www.snubi.org/software/ArrayXPath.

60. Huang, S., Mills, L., Mian, B., Tellez, C., McCarty, M., Yang, X.D., Gudas, J.M., Bar-Eli, M., 2002. Fully humanized neutralizing antibodies to interleukin-8 (ABXIL8) inhibit angiogenesis, tumor growth, and metastasis of human melanoma. Am J Pathol 161, 125-134.

61. IARC, 1987. IARC monographs on the evaluation of carcinogenic risks to humans. Overall evaluations of carcinogenicity to humans. International Agency for Research on Cancer. 32.

62. Ioannides, C., 1996. Cytochromes P450: Metabolic and Toxicological Aspects. Boca Raton, FL, CRC Press Inc.

63. Iruela-Arispe, M.L., Carpizo, D., Luque, A., 2003. ADAMTS1: a matrix metalloprotease with angioinhibitory properties. Ann N Y Acad Sci 995, 183-190.

64. Jackson, P.E., Kuang, S.Y., Wang, J.B., Strickland, P.T., Munoz, A., Kensler, T.W., Qian, G.S., Groopman, J.D., 2003. Prospective detection of codon 249 mutations in plasma of hepatocellular carcinoma patients. Carcinogenesis 24, 1657-1663.

65. Janig, G.R., Makower, A., Rabe, H., Bernhardt, R., Ruckpaul, K., 1984. Chemical modification of cytochrome P-450 LM2. Characterization of tyrosine as axial heme iron ligand trans to thiolate. Biochim Biophys Acta 787, 8-18.

66. Jeannin, P., Magistrelli, G., Aubry, J.P., Caron, G., Gauchat, J.F., Renno, T., Herbault, N., Goetsch, L., Blaecke, A., Dietrich, P.Y., Bonnefoy, J.Y., Delneste, Y., 2000. Soluble CD86 is a costimulatory molecule for human T lymphocytes. Immunity 13, 303-312.

67. Ji, Q., Aoyama, C., Nien, Y.D., Liu, P.I., Chen, P.K., Chang, L., Stanczyk, F.Z., Stolz, A., 2004. Selective loss of AKR1C1 and AKR1C2 in breast cancer and their potential effect on progesterone signaling. Cancer Res 64, 7610-7617.

68. Jiang, W.G., Watkins, G., Fodstad, O., Douglas-Jones, A., Mokbel, K., Mansel, R.E., 2004. Differential expression of the CCN family members Cyr61, CTGF and Nov in human breast cancer. Endocr Relat Cancer 11, 781-791.

69. John, K., Divi, R.L., Keshava, C., Orozco, C.C., Whipkey, D.L., Shockley, M., Poirier, M.C., Nath, J., Weston, A., Chlorophyllin modulates the expression of CYP1A1 and CYP1B1 and BP-DNA adduct formation in normal human mammary epithelial cells exposed to benzo(a)pyrene (in preparation).

70. Kamat, J.P., Boloor, K.K., Devasagayam, T.P., 2000. Chlorophyllin as an effective antioxidant against membrane damage in vitro and ex vivo. Biochim Biophys Acta 1487, 113-127.

71. Kazemi-Noureini, S., Colonna-Romano, S., Ziaee, A.A., Malboobi, M.A., Yazdanbod, M., Setayeshgar, P., Maresca, B., 2004. Differential gene expression between squamous cell carcinoma of esophageus and its normal epithelium; altered pattern of mal, akr1c2, and rab11a expression. World J Gastroenterol 10, 1716-1721.

72. Kennaway, E., 1955. The identification of a carcinogenic compound in coal-tar. $\mathrm{Br}$ Med J 749-752. 
73. Kensler, T.W., Egner, P.A., Wang, J.B., Zhu, Y.R., Zhang, B.C., Lu, P.X., Chen, J.G., Qian, G.S., Kuang, S.Y., Jackson, P.E., Gange, S.J., Jacobson, L.P., Munoz, A., Groopman, J.D., 2004. Chemoprevention of hepatocellular carcinoma in aflatoxin endemic areas. Gastroenterology 127, S310-318.

74. Kephart, J.C., 1955. Chlorophyll derivatives-their chemistry, commericial preparation and uses. Econ. Bot. 9, 3-38.

75. Keshava, C., Whipkey, D., Weston, A., 2005. Transcriptional signatures of environmentally relevant exposures in normal human mammary epithelial cells: benzo[a]pyrene. Cancer Lett 221, 201-211.

76. Kim, J.H., Stansbury, K.H., Walker, N.J., Trush, M.A., Strickland, P.T., Sutter, T.R., 1998. Metabolism of benzo[a]pyrene and benzo[a]pyrene-7,8-diol by human cytochrome P450 1B1. Carcinogenesis 19, 1847-1853.

77. Kirkman, H., 1959. Estrogen-induced tumors of the kidney. III. Growth characteristics in the Syrian hamster. Natl Cancer Inst Monogr 1, 1-57.

78. Knowles, M., Selby, P.E., 2005. Introduction to the Cellular and Molecular Biology of Cancer. NY, Oxford University Press Inc.

79. Kohler, C., Hakansson, A., Svanborg, C., Orrenius, S., Zhivotovsky, B., 1999. Protease activation in apoptosis induced by MAL. Exp Cell Res 249, 260-268.

80. Koo, J.H., Saraswati, M., Margolis, F.L., 2005. Immunolocalization of Bex protein in the mouse brain and olfactory system. J Comp Neurol 487, 1-14.

81. Kumar, S.S., Shankar, B., Sainis, K.B., 2004. Effect of chlorophyllin against oxidative stress in splenic lymphocytes in vitro and in vivo. Biochim Biophys Acta 1672, 100-111.

82. Laird, P.W., Zijderveld, A., Linders, K., Rudnicki, M.A., Jaenisch, R., Berns, A., 1991. Simplified mammalian DNA isolation procedure. Nucleic Acids Res 19, 4293.

83. Lam, G.K., Liao, H.X., Xue, Y., Alam, S.M., Scearce, R.M., Kaufman, R.E., Sempowski, G.D., Haynes, B.F., 2005. Expression of the CD7 ligand K-12 in human thymic epithelial cells: regulation by IFN-gamma. J Clin Immunol 25, 41-49.

84. Lampe, J.W., Stepaniants, S.B., Mao, M., Radich, J.P., Dai, H., Linsley, P.S., Friend, S.H., Potter, J.D., 2004. Signatures of environmental exposures using peripheral leukocyte gene expression: tobacco smoke. Cancer Epidemiol Biomarkers Prev 13, 445-453.

85. Layton, D.W., Bogen, K.T., Knize, M.G., Hatch, F.T., Johnson, V.M., Felton, J.S., 1995. Cancer risk of heterocyclic amines in cooked foods: an analysis and implications for research. Carcinogenesis 16, 39-52.

86. Lewis, D.F.V., 2001. Guide to cytochromes P450: Structure and Function. Taylor and Francis.

87. Liehr, J.G., Roy, D., 1990. Free radical generation by redox cycling of estrogens. Free Radic Biol Med 8, 415-423.

88. Lin, M.T., Zuon, C.Y., Chang, C.C., Chen, S.T., Chen, C.P., Lin, B.R., Wang, M.Y., Jeng, Y.M., Chang, K.J., Lee, P.H., Chen, W.J., Kuo, M.L., 2005. Cyr61 induces gastric cancer cell motility/invasion via activation of the integrin/nuclear factorkappaB/cyclooxygenase-2 signaling pathway. Clin Cancer Res 11, 5809-5820.

89. Livak, K.J., Schmittgen, T.D., 2001. Analysis of relative gene expression data using real-time quantitative PCR and the 2(-Delta Delta C(T)) Method. Methods 25, 402408. 
90. Lonning, P.E., Sorlie, T., Borresen-Dale, A.L., 2005. Genomics in breast cancertherapeutic implications. Nat Clin Pract Oncol 2, 26-33.

91. Ma, Q., Lu, A.Y., 2003. Origins of individual variability in P4501A induction. Chem Res Toxicol 16, 249-260.

92. Masson, L.F., Sharp, L., Cotton, S.C., Little, J., 2005. Cytochrome P-450 1A1 gene polymorphisms and risk of breast cancer: a HuGE review. Am J Epidemiol 161, 901915.

93. Mata, J.E., Yu, Z., Gray, J.E., Williams, D.E., Rodriguez-Proteau, R., 2004. Effects of chlorophyllin on transport of dibenzo(a, 1)pyrene, 2-amino-1-methyl-6phenylimidazo-[4,5-b]pyridine, and aflatoxin B(1) across Caco-2 cell monolayers. Toxicology 196, 117-125.

94. McKay, J.A., Melvin, W.T., Ah-See, A.K., Ewen, S.W., Greenlee, W.F., Marcus, C.B., Burke, M.D., Murray, G.I., 1995. Expression of cytochrome P450 CYP1B1 in breast cancer. FEBS Lett 374, 270-272.

95. Mian, B.M., Dinney, C.P., Bermejo, C.E., Sweeney, P., Tellez, C., Yang, X.D., Gudas, J.M., McConkey, D.J., Bar-Eli, M., 2003. Fully human anti-interleukin 8 antibody inhibits tumor growth in orthotopic bladder cancer xenografts via downregulation of matrix metalloproteases and nuclear factor-kappaB. Clin Cancer Res 9, 3167-3175.

96. Miller, E.C., 1951. Studies on the formation of protein-bound derivatives of 3,4benzpyrene in the epidermal fraction of mouse skin. Cancer Res 11, 100-108.

97. Negm, R.S., Verma, M., Srivastava, S., 2002. The promise of biomarkers in cancer screening and detection. Trends Mol Med 8, 288-293.

98. Negraes, P.D., Jordao, B.Q., Vicentini, V.E., Mantovani, M.S., 2004. Anticlastogenicity of chlorophyllin in the different cell cycle phases in cultured mammalian cells. Mutat Res 557, 177-182.

99. Nelson, R.L., 1992. Chlorophyllin, an antimutagen, acts as a tumor promoter in the rat-dimethylhydrazine colon carcinogenesis model. Anticancer Res 12, 737-739.

100. Oesch-Bartlomowicz, B., Huelster, A., Wiss, O., Antoniou-Lipfert, P., Dietrich, C., Arand, M., Weiss, C., Bockamp, E., Oesch, F., 2005. Aryl hydrocarbon receptor activation by cAMP vs. dioxin: divergent signaling pathways. Proc Natl Acad Sci U S A 102, 9218-9223.

101. Oesch-Bartlomowicz, B., Oesch, F., 2003. Cytochrome-P450 phosphorylation as a functional switch. Arch Biochem Biophys 409, 228-234.

102. Olivier, M., Hussain, S.P., Caron de Fromentel, C., Hainaut, P., Harris, C.C., 2004. TP53 mutation spectra and load: a tool for generating hypotheses on the etiology of cancer. IARC Sci Publ 247-270.

103. Ong, T.M., Whong, W.Z., Stewart, J., Brockman, H.E., 1986. Chlorophyllin: a potent antimutagen against environmental and dietary complex mixtures. Mutat Res 173, 111-115.

104. Osborne, M.R., Crosby, N.T., 1987. Benzopyrenes: Cambridge Monographs on Cancer Research. Cambridge University Press.

105. Pappa, A., Chen, C., Koutalos, Y., Townsend, A.J., Vasiliou, V., 2003. Aldh3a1 protects human corneal epithelial cells from ultraviolet- and 4-hydroxy-2-nonenalinduced oxidative damage. Free Radic Biol Med 34, 1178-1189. 
106. Park, K.K., Surh, Y.J., 1996. Chemopreventive activity of chlorophyllin against mouse skin carcinogenesis by benzo[a]pyrene and benzo[a]pyrene-7,8-dihydrodiol9,10-epoxide. Cancer Lett 102, 143-149.

107. Parkinson, A., 2003. Biotransformation of xenobiotics in Klaasen,C.D. and Watkins,J.B. (Eds.) Casarett and Doull's Essentials of toxicology. The McGraw-Hill Companies, Inc.

108. Pedersen, I.S., Dervan, P.A., Broderick, D., Harrison, M., Miller, N., Delany, E., O'Shea, D., Costello, P., McGoldrick, A., Keating, G., Tobin, B., Gorey, T., McCann, A., 1999. Frequent loss of imprinting of PEG1/MEST in invasive breast cancer. Cancer Res 59, 5449-5451.

109. Perera, F.P., Weinstein, I.B., 1982. Molecular epidemiology and carcinogen-DNA adduct detection: new approaches to studies of human cancer causation. J Chronic Dis 35, 581-600.

110. Perera, F.P., Weinstein, I.B., 2000. Molecular epidemiology: recent advances and future directions. Carcinogenesis 21, 517-524.

111. Phillips, D.H., 1983. Fifty years of benzo(a)pyrene. Nature 303, 468-472.

112. Poirier, M.C., 2004. Chemical-induced DNA damage and human cancer risk. Nat Rev Cancer 4, 630-637.

113. Porter, T.D., Coon, M.J., 1991. Cytochrome P-450. Multiplicity of isoforms, substrates, and catalytic and regulatory mechanisms. J Biol Chem 266, 13469-13472.

114. Puga, A., Tomlinson, C.R., Xia, Y., 2005. Ah receptor signals cross-talk with multiple developmental pathways. Biochem Pharmacol 69, 199-207.

115. Qiu, W., David, D., Zhou, B., Chu, P.G., Zhang, B., Wu, M., Xiao, J., Han, T., Zhu, Z., Wang, T., Liu, X., Lopez, R., Frankel, P., Jong, A., Yen, Y., 2003. Downregulation of growth arrest DNA damage-inducible gene 45beta expression is associated with human hepatocellular carcinoma. Am J Pathol 162, 1961-1974.

116. Quentmeier, H., Tonelli, R., Geffers, R., Pession, A., Uphoff, C.C., Drexler, H.G., 2005. Expression of BEX1 in acute myeloid leukemia with MLL rearrangements. Leukemia 19, 1488-1489.

117. Ramesh, A., Walker, S.A., Hood, D.B., Guillen, M.D., Schneider, K., Weyand, E.H., 2004. Bioavailability and risk assessment of orally ingested polycyclic aromatic hydrocarbons. Int J Toxicol 23, 301-333.

118. Reddy, A.P., Harttig, U., Barth, M.C., Baird, W.M., Schimerlik, M., Hendricks, J.D., Bailey, G.S., 1999. Inhibition of dibenzo[a,1]pyrene-induced multi-organ carcinogenesis by dietary chlorophyllin in rainbow trout. Carcinogenesis 20, 19191926.

119. Rodriguez, J.W., Kirlin, W.G., Wirsiy, Y.G., Matheravidathu, S., Hodge, T.W., Urso, P., 1999. Maternal exposure to benzo[a]pyrene alters development of T lymphocytes in offspring. Immunopharmacol Immunotoxicol 21, 379-396.

120. Rubin, H., 2001. Synergistic mechanisms in carcinogenesis by polycyclic aromatic hydrocarbons and by tobacco smoke: a bio-historical perspective with updates. Carcinogenesis 22, 1903-1930.

121. Sarkar, D., Sharma, A., Talukder, G., 1994. Chlorophyll and chlorophyllin as modifiers of genotoxic effects. Mutat Res 318, 239-247.

122. Sarkar, D., Sharma, A., Talukder, G., 1996. Clastogenic activity of pure chlorophyll and anticlastogenic effects of equivalent amounts of crude extract of Indian spinach 
leaf and chlorophyllin following dietary supplementation to mice. Environ Mol Mutagen 28, 121-126.

123. Saunders, D.B., Trapp, R.G., 1994. Association and prediction, in: J.Dolan,C. Lagan (Eds), Basic and Clinical biostatistics second ed. Appleton and Lange, Norwalk, CT. $162-187$.

124. Schmidt, J.V., Bradfield, C.A., 1996. Ah receptor signaling pathways. Annu Rev Cell Dev Biol 12, 55-89.

125. Schwarze, S.R., DePrimo, S.E., Grabert, L.M., Fu, V.X., Brooks, J.D., Jarrard, D.F., 2002. Novel pathways associated with bypassing cellular senescence in human prostate epithelial cells. J Biol Chem 277, 14877-14883.

126. Sharma, D., Kumar, S.S., Sainis, K.B., 2006. Antiapoptotic and immunomodulatory effects of chlorophyllin. Mol Immunol. Ahead of print.

127. Shellenberger, T.D., Wang, M., Gujrati, M., Jayakumar, A., Strieter, R.M., Burdick, M.D., Ioannides, C.G., Efferson, C.L., El-Naggar, A.K., Roberts, D., Clayman, G.L., Frederick, M.J., 2004. BRAK/CXCL14 is a potent inhibitor of angiogenesis and a chemotactic factor for immature dendritic cells. Cancer Res 64, 8262-8270.

128. Shen, A.L., Porter, T.D., Wilson, T.E., Kasper, C.B., 1989. Structural analysis of the FMN binding domain of NADPH-cytochrome P-450 oxidoreductase by site-directed mutagenesis. J Biol Chem 264, 7584-7589.

129. Shimada, T., Hayes, C.L., Yamazaki, H., Amin, S., Hecht, S.S., Guengerich, F.P., Sutter, T.R., 1996. Activation of chemically diverse procarcinogens by human cytochrome P-450 1B1. Cancer Res 56, 2979-2984.

130. Sims, P., Grover, P.L., Swaisland, A., Pal, K., Hewer, A., 1974. Metabolic activation of benzo(a)pyrene proceeds by a diol-epoxide. Nature 252, 326-328.

131. Singh, A., Singh, S.P., Bamezai, R., 1996. Modulatory influence of chlorophyllin on the mouse skin papillomagenesis and xenobiotic detoxication system. Carcinogenesis $17,1459-1463$.

132. Slentz-Kesler, K.A., Hale, L.P., Kaufman, R.E., 1998. Identification and characterization of K12 (SECTM1), a novel human gene that encodes a Golgiassociated protein with transmembrane and secreted isoforms. Genomics 47, 327340.

133. Smith, S.D., Wheeler, M.A., Plescia, J., Colberg, J.W., Weiss, R.M., Altieri, D.C., 2001. Urine detection of survivin and diagnosis of bladder cancer. Jama 285, 324328.

134. Sokal, R.R., Michener, C.D., 1958. A statistical method for evaluating systematic relationships Univ. of Kans. Sci. Bull. 38 1409-1438.

135. Spink, D.C., Spink, B.C., Cao, J.Q., DePasquale, J.A., Pentecost, B.T., Fasco, M.J., Li, Y., Sutter, T.R., 1998. Differential expression of CYP1A1 and CYP1B1 in human breast epithelial cells and breast tumor cells. Carcinogenesis 19, 291-298.

136. Srivastava, S., Gopal-Srivastava, R., 2002. Biomarkers in cancer screening: a public health perspective. J Nutr 132, 2471S-2475S.

137. Stampfer, M., Hallowes, R.C., Hackett, A.J., 1980. Growth of normal human mammary cells in culture. In Vitro 16, 415-425.

138. Sterling, K., Raha, A., Bresnick, E., 1994. Induction of CYP1A1 gene expression in mouse hepatoma cells by benzo[e]pyrene, a ligand of the 4S polycyclic hydrocarbonbinding protein. Toxicol Appl Pharmacol 128, 18-24. 
139. Sudakin, D.L., 2003. Dietary aflatoxin exposure and chemoprevention of cancer: a clinical review. J Toxicol Clin Toxicol 41, 195-204.

140. Suomela, S., Cao, L., Bowcock, A., Saarialho-Kere, U., 2004. Interferon alphainducible protein 27 (IFI27) is upregulated in psoriatic skin and certain epithelial cancers. J Invest Dermatol 122, 717-721.

141. Suvas, S., Singh, V., Sahdev, S., Vohra, H., Agrewala, J.N., 2002. Distinct role of $\mathrm{CD} 80$ and CD86 in the regulation of the activation of B cell and B cell lymphoma. J Biol Chem 277, 7766-7775.

142. Tachino, N., Guo, D., Dashwood, W.M., Yamane, S., Larsen, R., Dashwood, R., 1994. Mechanisms of the in vitro antimutagenic action of chlorophyllin against benzo[a]pyrene: studies of enzyme inhibition, molecular complex formation and degradation of the ultimate carcinogen. Mutat Res 308, 191-203.

143. Terwel, L., van der Hoeven, J.C., 1985. Antimutagenic activity of some naturally occurring compounds towards cigarette-smoke condensate and benzo[a]pyrene in the Salmonella/microsome assay. Mutat Res 152, 1-4.

144. Tobioka, H., Isomura, H., Kokai, Y., Tokunaga, Y., Yamaguchi, J., Sawada, N., 2004. Occludin expression decreases with the progression of human endometrial carcinoma. Hum Pathol 35, 159-164.

145. Torres-Bezauri, R., Madrigal-Bujaidar, E., Alvarez-Gonzalez, R.I., Zepeda, G., Chamorro, G., 2002. Effects of chlorophyllin on acetaldehyde: lack of modulation of the rate of sister-chromatid exchanges in mouse bone marrow, and of complex formation in aqueous solution. Food Chem Toxicol 40, 1507-1513.

146. Vrzal, R., Ulrichova, J., Dvorak, Z., 2004. Aromatic hydrocarbon receptor status in the metabolism of xenobiotics under normal and pathophysiological conditions. Biomed Pap Med Fac Univ Palacky Olomouc Czech Repub 148, 3-10.

147. Warner, J.R., Nath, J., Ong, T.M., 1991. Antimutagenicity studies of chlorophyllin using the Salmonella arabinose-resistant assay system. Mutat Res 262, 25-30.

148. Waters, M.D., Stack, H.F., Jackson, M.A., Brockman, H.E., De Flora, S., 1996. Activity profiles of antimutagens: in vitro and in vivo data. Mutat Res 350, 109-129.

149. Wattenberg, L.W., 1966. Chemoprophylaxis of carcinogenesis: a review. Cancer Res 26, 1520-1526.

150. Wattenberg, L.W., 1997. An overview of chemoprevention: current status and future prospects. Proc Soc Exp Biol Med 216, 133-141.

151. Weston, A., Harris, C.C., 2005. Chemical carcinogenesis, in: Cancer Medicine, seventh eds., B.C. Decker Inc., Hamilton,ON. Eds. J. F. Holland, E. Frei, III. pp246258. 267-278.

152. Weston, A., Poirier, M.C., 2002. DNA damage, DNA repair, and mutagenesis;in Encyclopedia of Cancer, Second edition, Academic Press, San Diego, CA. Ed. J.R. Bertino. Volume1:pp.641-649.

153. Whitlock, J.P., Jr., 1999. Induction of cytochrome P4501A1. Annu Rev Pharmacol Toxicol 39, 103-125.

154. Wogan, G.N., Hecht, S.S., Felton, J.S., Conney, A.H., Loeb, L.A., 2004. Environmental and chemical carcinogenesis. Semin Cancer Biol 14, 473-486.

155. Wu, Q., Ding, W., Mirza, A., Van Arsdale, T., Wei, I., Bishop, W.R., Basso, A., McClanahan, T., Luo, L., Kirschmeier, P., Gustafson, E., Hernandez, M., Liu, S., 
2005. Integrative genomics revealed RAI3 is a cell growth-promoting gene and a novel P53 transcriptional target. J Biol Chem 280, 12935-12943.

156. Yamagami, H., Yamagami, S., Inoki, T., Amano, S., Miyata, K., 2003. The effects of proinflammatory cytokines on cytokine-chemokine gene expression profiles in the human corneal endothelium. Invest Ophthalmol Vis Sci 44, 514-520.

157. Yamamoto, S., Egami, H., Kurizaki, T., Ohmachi, H., Hayashi, N., Okino, T., Shibata, Y., Schalkwijk, J., Ogawa, M., 1997. Immunohistochemical expression of SKALP/elafin in squamous cell carcinoma of the oesophagus. Br J Cancer 76, 10811086.

158. Yamamoto, T., Akisue, T., Marui, T., Nakatani, T., Kawamoto, T., Hitora, T., Nagira, K., Fujita, I., Matsumoto, K., Kurosaka, M., 2004. Expression of betacellulin, heparin-binding epidermal growth factor and epiregulin in human malignant fibrous histiocytoma. Anticancer Res 24, 2007-2010.

159. Yan, C., Lu, D., Hai, T., Boyd, D.D., 2005. Activating transcription factor 3, a stress sensor, activates p53 by blocking its ubiquitination. Embo J 24, 2425-2435.

160. Yun, C.H., Son, C.G., Chung, D.K., Han, S.H., 2005. Chlorophyllin attenuates IFNgamma expression in lipopolysaccharide-stimulated murine splenic mononuclear cells via suppressing IL-12 production. Int Immunopharmacol 5, 1926-1935.

161. Yuspa, S.H., Shields, P.G., 1997. Etiology of Cancers:Chemical Factors in Cancer. in Principles and Practice of Oncology, DeVita,V.T., Hellman,Jr.S. and Rosenberg,S.A. (Eds) Lippincott-Raven Publishers, Philadelphia.

162. Zella, D., Barabitskaja, O., Casareto, L., Romerio, F., Secchiero, P., Reitz, M.S., Jr., Gallo, R.C., Weichold, F.F., 1999. Recombinant IFN-alpha (2b) increases the expression of apoptosis receptor CD95 and chemokine receptors CCR1 and CCR3 in monocytoid cells. J Immunol 163, 3169-3175.

163. Zhao, C., Denison, C., Huibregtse, J.M., Gygi, S., Krug, R.M., 2005. Human ISG15 conjugation targets both IFN-induced and constitutively expressed proteins functioning in diverse cellular pathways. Proc Natl Acad Sci U S A 102, 1020010205. 


\subsection{APPENDIX}

Tissue Mix media

Penicillin Streptomycin mix (10,000U/ml Penicillin G sodium and $10,000 \mathrm{U} / \mathrm{ml}$ Streptomycin sulfate) $\quad 10 \mathrm{ml}$ Fungizone $(250 \mu \mathrm{g} / \mathrm{ml}$ Amphothericin and $250 \mu \mathrm{g} / \mathrm{ml}$ sodium deoxycholate $) \quad 20 \mathrm{ml}$ Insulin $(10 \mathrm{mg} / \mathrm{ml})$ $1 \mathrm{ml}$ Hanks buffered saline $\underline{969 \mathrm{ml}}$

Total $1000 \mathrm{ml}$

Filter through $0.2 \mu \mathrm{M}$ filter.

Tissue Digestion media

Tissue mix media

$70 \mathrm{ml}$

Collagenase $(2000 \mathrm{U} / \mathrm{ml})$

$10 \mathrm{ml}$

Hyaluronidase $10 \mathrm{ml}$

Fetal Bovine Serum $10 \mathrm{ml}$

Total

$100 \mathrm{ml}$

Filter through $0.2 \mu \mathrm{M}$ filter.

$\underline{\text { Freezing media }}$

MEBM media

$80 \mathrm{ml}$

DMSO

$10 \mathrm{ml}$

Fetal Bovine Serum

$\underline{10 \mathrm{ml}}$

Total

$100 \mathrm{ml}$

Filter through $0.2 \mu \mathrm{M}$ filter.

MCF-7 media

DMEM (Dulbecco's modified Eagle's medium)

$894.5 \mathrm{ml}$

Fetal calf serum (FCS)*

$100 \mathrm{ml}$

Penicillin (5000U/ml)-Streptomycin mix $(5000 \mu \mathrm{g} / \mathrm{ml}) \mathrm{mix}$

$2 \mathrm{ml}$

Amphothericin B $(250 \mu \mathrm{g} / \mathrm{ml})$

$1 \mathrm{ml}$

Insulin $(1000 \mu \mathrm{g} / \mathrm{ml})$

Total

$\frac{2.5 \mathrm{ml}}{1000 \mathrm{ml}}$

Filter through $0.2 \mu \mathrm{M}$ filter.

* FCS is to be heated $\left(56^{\circ} \mathrm{C}\right.$ for $\left.50 \mathrm{~min}\right)$ to inactivate components of the complement system

5X RNA Fragmentation buffer

$200 \mathrm{mM}$ Tris acetate, $\mathrm{pH} 8.1$

$500 \mathrm{mM}$ potassium acetate

$150 \mathrm{mM}$ magnesium acetate 